\title{
Silver-Catalyzed Decarboxylative Allylation of Aliphatic Carboxylic Acids in Aqueous Solution
}

\author{
Lei Cui, ${ }^{\mathrm{a}} \mathrm{He}$ Chen, ${ }^{\mathrm{b}}$ Chao Liu, ${ }^{\mathrm{b}}$ and Chaozhong Li*, c \\ ${ }^{a}$ Department of Chemistry, University of Science and Technology of China, Hefei, Anhui 230026, \\ China \\ ${ }^{b}$ Key Laboratory of Organofluorine Chemistry and Collaborative Innovation Center of Chemistry \\ for Life Sciences, Shanghai Institute of Organic Chemistry, Chinese Academy of Sciences, 345 \\ Lingling Road, Shanghai 200032, China \\ ${ }^{c}$ School of Chemical Engineering, Ningbo University of Technology, No. 89 Cuibai Road, \\ Ningbo 315016, China
}

clig@mail.sioc.ac.cn

\section{Table of Contents}

1. Characterizations of New Substrates.

S2

2. Typical Procesure for Silver-Catalyzed Decarboxylative Allylation

S2

3. Characterizations of Products.

4. References for Known Compounds.

S14

5. ${ }^{1} \mathrm{H}$ and ${ }^{13} \mathrm{C}$ NMR Spectra of Substrate Acids and Allylating Agents

S15-S45

6. ${ }^{1} \mathrm{H}$ and ${ }^{13} \mathrm{C}$ NMR Spectra of Products

S46-S79 


\section{Characterizations of New Substrates.}<smiles>CC(CN1C(=O)c2ccccc2C1=O)C(=O)O</smiles>

3-(1,3-Dioxoisoindolin-2-yl)-2-methylpropanoic acid. 3-Amino-2-methylpropanoic acid (2.06 g, $20 \mathrm{mmol})$, phthalic anhydride (4.08 g, $20 \mathrm{mmol})$, toluene $(45 \mathrm{~mL})$, and $\mathrm{Et}_{3} \mathrm{~N}(0.28 \mathrm{~mL}, 2.0 \mathrm{mmol})$ were added to a $100 \mathrm{~mL}$ round bottom flask. After refluxing the reaction mixture overnight, concentrated hydrochloric acid $(0.4 \mathrm{~mL})$ and water $(50 \mathrm{~mL})$ were added into the solution. The resulting mixture was extracted with ethyl acetate and the organic layer was dried over anhydrous $\mathrm{Na}_{2} \mathrm{SO}_{4}$. The organic solvent was removed and the residue was recrystallized by $\mathrm{MeOH} / \mathrm{H}_{2} \mathrm{O}$ to give $\mathbf{1 h}$ as a white solid. Yield: $3.26 \mathrm{~g}$ (70\%).Mp: $158-160{ }^{\circ} \mathrm{C} ;{ }^{1} \mathrm{H}$ NMR $\left(400 \mathrm{MHz}, \mathrm{CDCl}_{3}\right): \delta$ 7.88-7.84 (m, 2H), 7.75-7.71 (m, 2H), $4.01(\mathrm{dd}, J=7.2,14.0 \mathrm{~Hz}, 1 \mathrm{H}), 3.78$ (dd, $J=$ 7.2, $14.0 \mathrm{~Hz}, 1 \mathrm{H}), 3.10-3.01(\mathrm{~m}, 1 \mathrm{H}), 1.23(\mathrm{~d}, J=6.8 \mathrm{~Hz}, 3 \mathrm{H}) ;{ }^{13} \mathrm{C}$ NMR $(100 \mathrm{MHz}$, $\left.\mathrm{CDCl}_{3}\right): \delta 179.0,168.2,134.1,131.9,123.4,40.2,38.1,14.6$; IR $(\mathrm{KBr}): v\left(\mathrm{~cm}^{-1}\right) 1773$, 1709, 1466, 1436, 1396, 1357, 1321, 1239, 1031, 936, 724; ESI-MS: $(\mathrm{m} / \mathrm{z}) 256.1$ $\left(\mathrm{M}^{+}+\mathrm{Na}\right)$; HRMS calcd for $\mathrm{C}_{12} \mathrm{H}_{12} \mathrm{O}_{4} \mathrm{~N}(\mathrm{M}+\mathrm{H}): 234.0761$, found 234.0759.<smiles>Cc1ccc(S(=O)(=O)C(F)C=O)cc1</smiles>

1-((1-Fluoroallyl)sulfonyl)-4-methylbenzene. 1-(Allylsulfonyl)-4-methylbenzene $(1.96 \mathrm{~g}, 10 \mathrm{mmol})$ and THF $(20 \mathrm{~mL})$ were added to a $50 \mathrm{~mL}$ round bottom flask. A solution of $n$-BuLi in THF $(2.5 \mathrm{M}, 4.8 \mathrm{~mL}, 12 \mathrm{mmol})$ was added drapwise at $-78{ }^{\circ} \mathrm{C}$. NFSI (4.72 g, $15 \mathrm{mmol}$ ) was added after $0.5 \mathrm{~h}$. The reaction mixture was stired overnight at $\mathrm{rt}$, and then saturated $\mathrm{NH}_{4} \mathrm{Cl}$ solution $(10 \mathrm{~mL})$ was added. The resulting mixture was extracted with ethyl acetate and the organic layer was dried over anhydrous $\mathrm{Na}_{2} \mathrm{SO}_{4}$. After removal of the solvent under reduced pressure, the crude product was purified by column chromatography on silica gel with hexane/ethyl acetate $(4: 1, \mathrm{v}: \mathrm{v})$ as the eluent to give the pure $2 \mathrm{e}$ as a colorless oil. Yield: $0.43 \mathrm{~g}$ (20\%). ${ }^{1} \mathrm{H}$ NMR (400 MHz, $\left.\mathrm{CDCl}_{3}\right): \delta 7.79$ (d, $\left.J=8.4 \mathrm{~Hz}, 2 \mathrm{H}\right), 7.38(\mathrm{~d}, J=8.0 \mathrm{~Hz}$, $2 \mathrm{H}), 6.04-5.91(\mathrm{~m}, 1 \mathrm{H}), 5.62-5.48(\mathrm{~m}, 3 \mathrm{H}), 2.46(\mathrm{~s}, 3 \mathrm{H}) ;{ }^{13} \mathrm{C}$ NMR $(100 \mathrm{MHz}$, $\left.\mathrm{CDCl}_{3}\right): \delta 146.0,131.7,129.9,129.8,125.3(\mathrm{~d}, J=19.0 \mathrm{~Hz}), 124.7(\mathrm{~d}, J=10.9 \mathrm{~Hz})$, $101.9(\mathrm{~d}, J=218.0 \mathrm{~Hz}), 21.7 ;{ }^{19} \mathrm{~F}$ NMR $\left(376 \mathrm{MHz}, \mathrm{CDCl}_{3}\right): \delta-174.1(\mathrm{dd}, J=46.6$, $16.5 \mathrm{~Hz}, 1 \mathrm{~F})$; IR (neat): $v\left(\mathrm{~cm}^{-1}\right)$ 1596, 1494, 1329, 1231, 1154, 1123, 1044, 947, 817, 706, 654, 585; EIMS: $m / z$ (rel intensity) $214\left(\mathrm{M}^{+}, 0.3\right), 155$ (95), 149 (18), 139 (16), 118 (9), 92 (11), 91 (100), 89 (9), 65 (23), 59 (39); HRMS calcd for $\mathrm{C}_{10} \mathrm{H}_{11} \mathrm{FO}_{2} \mathrm{~S}(\mathrm{M})$ : 214.0464, found 214.0458. 


\section{Typical Procedure for Silver-Catalyzed Decarboxylative Allylation}

2-Ethyltetradecanoic acid (1a, $51.2 \mathrm{mg}, 0.20 \mathrm{mmol}), \mathrm{AgNO}_{3}(6.8 \mathrm{mg}, 0.04 \mathrm{mmol})$, $\mathrm{K}_{2} \mathrm{~S}_{2} \mathrm{O}_{8}(81 \mathrm{mg}, 0.30 \mathrm{mmol})$ and ethyl 2-(tosylmethyl)acrylate (2a, $107 \mathrm{mg}, 0.40$ mmol) were placed in a Schlenk tube. Acetonitrile $(1 \mathrm{~mL})$ and water $(1 \mathrm{~mL})$ were then added under nitrogen atmosphere. The solution was stirred at $50{ }^{\circ} \mathrm{C}$ for $12 \mathrm{~h}$. The resulting mixture was cooled down to room temperature and extracted with diethyl ether $(5 \mathrm{~mL} \times 4)$. The combined organic phase was dried over anhydrous $\mathrm{Na}_{2} \mathrm{SO}_{4}$. After the removal of solvent under reduced pressure, the crude product was purified by column chromatography on silica gel with hexane as the eluent to give the pure product ethyl 4-ethyl-2-methylenehexadecanoate (3a) as colorless oil. Yield: $57.7 \mathrm{mg}$ (89\%).

\section{Characterizations of Products.}<smiles>C=C(CC(CC)CC)C(=O)OCC</smiles>

Ethyl 4-ethyl-2-methylenehexadecanoate (3a). Colorless oil $(57.7 \mathrm{mg}, 89 \%) ;{ }^{1} \mathrm{H}$ NMR (400 MHz, $\left.\mathrm{CDCl}_{3}\right): \delta 6.15(\mathrm{~d}, J=2.0 \mathrm{~Hz}, 1 \mathrm{H}), 5.46(\mathrm{~d}, J=1.2 \mathrm{~Hz}, 1 \mathrm{H}), 4.20(\mathrm{q}$, $J=7.2 \mathrm{~Hz}, 2 \mathrm{H}), 2.22(\mathrm{~d}, J=6.8 \mathrm{~Hz}, 2 \mathrm{H}), 1.51-1.42(\mathrm{~m}, 1 \mathrm{H}), 1.32-1.25(\mathrm{~m}, 27 \mathrm{H})$, 0.90-0.84 (m, 6H); ${ }^{13} \mathrm{C}$ NMR $\left(100 \mathrm{MHz}, \mathrm{CDCl}_{3}\right): \delta 167.6,140.2,125.5,60.5,37.9$, $36.4,32.7,31.9,30.0,29.7,29.3,26.5,25.5,22.7,14.2,14.1,10.6$; IR (neat): $v\left(\mathrm{~cm}^{-1}\right)$ 2958, 2925, 2854, 1721, 1630, 1464, 1324, 1301, 1176, 1155, 1029, 934, 817; EIMS: m/z (rel intensity) $324\left(\mathrm{M}^{+}, 5\right), 295$ (18), 236 (6), 223 (9), 180 (15), 154 (36), 114 (100), 86 (19), 69 (17); HRMS calcd for $\mathrm{C}_{21} \mathrm{H}_{40} \mathrm{O}_{2}(\mathrm{M})$ : 324.3028 , found 324.3022.

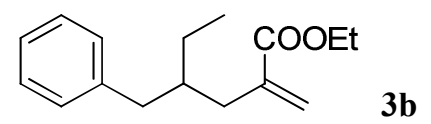

Ethyl 4-benzyl-2-methylenehexanoate (3b). Colorless oil (35.0 mg, 71\%); ${ }^{1} \mathrm{H}$ NMR $\left(400 \mathrm{MHz}, \mathrm{CDCl}_{3}\right): \delta 7.26(\mathrm{t}, J=7.2 \mathrm{~Hz}, 2 \mathrm{H}), 7.18-7.13(\mathrm{~m}, 3 \mathrm{H}), 6.18(\mathrm{~d}, J=1.6 \mathrm{~Hz}$, $1 \mathrm{H}), 5.49$ (s, $J=1.2 \mathrm{~Hz}, 1 \mathrm{H}), 4.17$ (q, $J=7.2 \mathrm{~Hz}, 2 \mathrm{H}), 2.59-2.49(\mathrm{~m}, 2 \mathrm{H}), 2.34-2.21$ $(\mathrm{m}, 2 \mathrm{H}), 1.89-1.79(\mathrm{~m}, 1 \mathrm{H}), 1.35-1.25(\mathrm{~m}, 5 \mathrm{H}), 0.89(\mathrm{t}, J=7.2 \mathrm{~Hz}, 3 \mathrm{H}) ;{ }^{13} \mathrm{C}$ NMR $\left(100 \mathrm{MHz}, \mathrm{CDCl}_{3}\right): \delta 167.4,141.1,139.9,129.2,128.1,125.9,125.7,60.6,40.0,39.7$, 36.1, 25.1, 14.2, 10.6; IR (neat): $v\left(\mathrm{~cm}^{-1}\right)$ 3062, 3027, 2962, 2928, 2875, 1717, 1629, 1496, 1454, 1368, 1303, 1184, 1147, 1029, 942, 817, 741, 700; ESI-MS: $(\mathrm{m} / z) 247.0$ $\left(\mathrm{M}^{+}+\mathrm{H}\right)$; HRMS calcd for $\mathrm{C}_{16} \mathrm{H}_{23} \mathrm{O}_{2}(\mathrm{M}+\mathrm{H}): 247.1693$, found 247.1693. 
<smiles>C=C(CC(CC)CCCCCBr)C(=O)OCC</smiles>

Ethyl 9-bromo-4-ethyl-2-methylenenonanoate (3c). Colorless oil (44.0 mg, 72\%); ${ }^{1} \mathrm{H}$ NMR (400 MHz, $\left.\mathrm{CDCl}_{3}\right): \delta 6.16(\mathrm{~d}, J=1.2 \mathrm{~Hz}, 1 \mathrm{H}), 5.48(\mathrm{~s}, 1 \mathrm{H}), 4.20$ (q, $J=7.2$ $\mathrm{Hz}, 2 \mathrm{H}), 3.40$ (t, $J=6.8 \mathrm{~Hz}, 2 \mathrm{H}), 2.23$ (d, $J=7.2 \mathrm{~Hz}, 2 \mathrm{H}), 1.89-1.82(\mathrm{~m}, 2 \mathrm{H})$, $1.50-1.21(\mathrm{~m}, 12 \mathrm{H}), 0.86(\mathrm{t}, J=7.6 \mathrm{~Hz}, 3 \mathrm{H}) ;{ }^{13} \mathrm{C} \mathrm{NMR}\left(100 \mathrm{MHz}, \mathrm{CDCl}_{3}\right): \delta 167.5$, $140.0,125.6,60.5,37.8,36.4,34.0,32.8,32.5,28.5,25.7,25.5,14.2,10.6$; IR (neat): $v\left(\mathrm{~cm}^{-1}\right)$ 2961, 2932, 2858, 1717, 1630, 1462, 1368, 1324, 1301, 1177, 1158, 1028, 942, 818; ESI-MS: $(m / z)$ 327.0/329.1 $\left(\mathrm{M}^{+}+\mathrm{Na}\right)$; HRMS calcd for $\mathrm{C}_{14} \mathrm{H}_{26} \mathrm{BrO}_{2}(\mathrm{M}+\mathrm{H})$ : 305.1111, found 305.1110 .<smiles>C=C(CC(Cc1ccc(Cl)cc1)Cc1ccc(Cl)cc1)C(=O)OCC</smiles>

Ethyl 4,5-di(4-chlorobenzyl)-2-methylenepentanoate (3d). Colorless oil (58.9 mg, 78\%); ${ }^{1} \mathrm{H}$ NMR (400 MHz, $\left.\mathrm{CDCl}_{3}\right): \delta 7.22(\mathrm{~d}, J=8.4 \mathrm{~Hz}, 4 \mathrm{H}), 7.03(\mathrm{~d}, J=8.4 \mathrm{~Hz}$, $4 \mathrm{H}), 6.20(\mathrm{~d}, J=1.2 \mathrm{~Hz}, 1 \mathrm{H}), 5.50(\mathrm{~s}, 1 \mathrm{H}), 4.14(\mathrm{q}, J=7.2 \mathrm{~Hz}, 2 \mathrm{H}), 2.54-2.43(\mathrm{~m}$, $4 \mathrm{H}), 2.25-2.14(\mathrm{~m}, 3 \mathrm{H}), 1.23(\mathrm{t}, J=7.2 \mathrm{~Hz}, 3 \mathrm{H}) ;{ }^{13} \mathrm{C} \mathrm{NMR}\left(100 \mathrm{MHz}, \mathrm{CDCl}_{3}\right): \delta$ 167.0, 139.2, 138.9, 131.7, 130.5, 128.4, 126.7, 60.7, 40.6, 39.3, 36.5, 14.1; IR (neat): $v\left(\mathrm{~cm}^{-1}\right)$ 3026, 2981, 2927, 2855, 1716, 1628, 1492, 1445, 1408, 1302, 1187, 1138, 1090, 1015, 818; EIMS: $m / z$ (rel intensity) $376\left(\mathrm{M}^{+}, 36\right), 262$ (30), 227 (26), 205 (37), 177 (31), 142 (17), 125 (100), 115 (15); HRMS calcd for $\mathrm{C}_{21} \mathrm{H}_{22} \mathrm{O}_{2} \mathrm{Cl}_{2}$ (M): 376.0997, found 376.0995 .

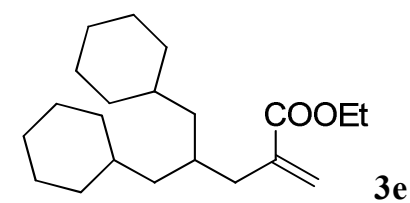

Ethyl 5-cyclohexyl-4-(cyclohexylmethyl)-2-methylenepentanoate (3e). Colorless oil (42.3 mg, 66\%); ${ }^{1} \mathrm{H}$ NMR (400 MHz, $\left.\mathrm{CDCl}_{3}\right): \delta 6.14(\mathrm{~s}, 1 \mathrm{H}), 5.44(\mathrm{~s}, 1 \mathrm{H}), 4.20(\mathrm{q}$, $J=7.2 \mathrm{~Hz}, 2 \mathrm{H}), 2.18(\mathrm{~d}, J=6.4 \mathrm{~Hz}, 2 \mathrm{H}), 1.78-1.62(\mathrm{~m}, 10 \mathrm{H}), 1.38-0.94(\mathrm{~m}, 16 \mathrm{H})$, 0.88-0.74 (m, 4H); ${ }^{13} \mathrm{C}$ NMR (100 MHz, $\left.\mathrm{CDCl}_{3}\right): \delta 167.5,140.1,125.7,60.5,42.5$, 37.9, 34.7, 33.6, 30.0, 26.7, 26.4, 14.2; IR (neat): $v\left(\mathrm{~cm}^{-1}\right)$ 2921, 2850, 1719, 1629, 1448, 1181, 1029, 940, 750; ESI-MS: $(\mathrm{m} / z) 321.2\left(\mathrm{M}^{+}+\mathrm{H}\right)$; HRMS calcd for $\mathrm{C}_{21} \mathrm{H}_{37} \mathrm{O}_{2}$ $(\mathrm{M}+\mathrm{H}): 321.2788$, found 321.2786 .<smiles>C=C(CC1CCCCC1)C(=O)OCC</smiles>

Ethyl 2-(cyclohexylmethyl)acrylate (3f). Colorless oil (26.7 mg, 68\%); ${ }^{1} \mathrm{H}$ NMR 
$\left(400 \mathrm{MHz}, \mathrm{CDCl}_{3}\right): \delta 6.14(\mathrm{~d}, J=1.2 \mathrm{~Hz}, 1 \mathrm{H}), 5.46(\mathrm{~s}, 1 \mathrm{H}), 4.20(\mathrm{q}, J=7.2 \mathrm{~Hz}, 2 \mathrm{H})$, $2.18(\mathrm{~d}, J=6.8 \mathrm{~Hz}, 2 \mathrm{H}), 1.71-1.62(\mathrm{~m}, 5 \mathrm{H}), 1.51-1.37(\mathrm{~m}, 1 \mathrm{H}), 1.32-1.06(\mathrm{~m}, 6 \mathrm{H})$, 0.93-0.80 (m, 2H); ${ }^{13} \mathrm{C}$ NMR (100 MHz, $\left.\mathrm{CDCl}_{3}\right): \delta 167.5,139.4,125.4,60.5,39.9$, 36.6, 33.0, 26.5, 26.2, 14.2; IR (neat): $v\left(\mathrm{~cm}^{-1}\right)$ 2925, 2853, 1721, 1631, 1462, 1449, 1261, 1179, 1156, 1096, 1026, 803; ESI-MS: $(\mathrm{m} / \mathrm{z}) 219.1\left(\mathrm{M}^{+}+\mathrm{Na}\right)$; HRMS calcd for $\mathrm{C}_{12} \mathrm{H}_{21} \mathrm{O}_{2}(\mathrm{M}+\mathrm{H}):$ 197.1536, found 197.1535.

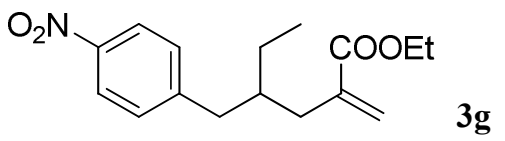

Ethyl 2-methylene-4-(4-nitrobenzyl)hexanoate (3g). Colorless oil (40.2 mg, 69\%); ${ }^{1} \mathrm{H}$ NMR (400 MHz, $\mathrm{CDCl}_{3}$ ): $\delta 8.14(\mathrm{~d}, J=8.8 \mathrm{~Hz}, 2 \mathrm{H}), 7.31(\mathrm{~d}, J=8.8 \mathrm{~Hz}, 2 \mathrm{H}), 6.21$ $(\mathrm{d}, J=1.2 \mathrm{~Hz}, 1 \mathrm{H}), 5.53(\mathrm{~d}, J=1.2 \mathrm{~Hz}, 1 \mathrm{H}), 4.19$ (q, $J=7.2 \mathrm{~Hz}, 2 \mathrm{H}), 2.70-2.61(\mathrm{~m}$, 2H), $2.34(\mathrm{dd}, J=7.2,14.0 \mathrm{~Hz}, 1 \mathrm{H}), 2.22(\mathrm{dd}, J=6.8,14.0 \mathrm{~Hz}, 1 \mathrm{H}), 1.96-1.86(\mathrm{~m}$, 1H), $1.34-1.24(\mathrm{~m}, 5 \mathrm{H}), 0.91(\mathrm{t}, J=7.2 \mathrm{~Hz}, 3 \mathrm{H}) ;{ }^{13} \mathrm{C}$ NMR $\left(100 \mathrm{MHz}, \mathrm{CDCl}_{3}\right): \delta$ 167.1, 149.4, 146.3, 139.3, 129.9, 126.5, 123.4, 60.7, 39.9, 39.7, 36.3, 25.2, 14.2, 10.6; IR (neat): $v\left(\mathrm{~cm}^{-1}\right)$ 2963, 2932, 2876, 1715, 1629, 1598, 1519, 1346, 1185, 1148, 1027 , 946, 855, 744, 701; ESI-MS: $(\mathrm{m} / \mathrm{z}) 314.0\left(\mathrm{M}^{+}+\mathrm{Na}\right)$; HRMS calcd for $\mathrm{C}_{16} \mathrm{H}_{22} \mathrm{O}_{4} \mathrm{~N}$ $(\mathrm{M}+\mathrm{H}): 292.1543$, found 292.1540 .

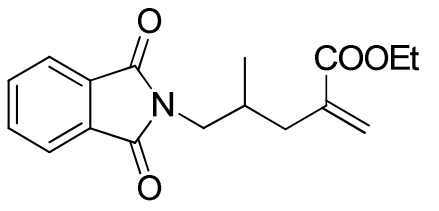

$3 \mathrm{~h}$

Ethyl 5-(1,3-dioxoisoindolin-2-yl)-4-methyl-2-methylenepentanoate (3h). Colorless oil (42.2 mg, 70\%); ${ }^{1} \mathrm{H}$ NMR (400 MHz, $\left.\mathrm{CDCl}_{3}\right): \delta 7.87-7.83(\mathrm{~m}, 2 \mathrm{H})$, 7.74-7.71 (m, 2H), $6.19(\mathrm{~d}, J=1.2 \mathrm{~Hz}, 1 \mathrm{H}), 5.59(\mathrm{~s}, 1 \mathrm{H}), 4.18-4.13(\mathrm{~m}, 2 \mathrm{H}), 3.62(\mathrm{dd}$, $J=7.2,13.6 \mathrm{~Hz}, 1 \mathrm{H}), 3.54$ (dd, $J=7.6,13.6 \mathrm{~Hz}, 1 \mathrm{H}), 2.45$ (dd, $J=5.6,14.0 \mathrm{~Hz}, 1 \mathrm{H}$ ), 2.33-2.21 (m, 1H), 2.13 (dd, $J=8.4,13.6 \mathrm{~Hz}, 1 \mathrm{H}), 1.24$ (t, $J=7.2 \mathrm{~Hz}, 3 \mathrm{H}), 0.91$ (d, $J$ $=6.8 \mathrm{~Hz}, 3 \mathrm{H}) ;{ }^{13} \mathrm{C} \mathrm{NMR}\left(100 \mathrm{MHz}, \mathrm{CDCl}_{3}\right): \delta 168.6,167.0,138.6,133.9,132.0$, 126.4, 123.2, 60.6, 43.8, 37.1, 31.5, 17.3, 14.1; IR (neat): $v\left(\mathrm{~cm}^{-1}\right)$ 2964, 2931, 1773, 1712, 1630, 1467, 1434, 1397, 1177, 1046, 724; ESI-MS: $(\mathrm{m} / \mathrm{z}) 324.1\left(\mathrm{M}^{+}+\mathrm{Na}\right)$; HRMS calcd for $\mathrm{C}_{17} \mathrm{H}_{20} \mathrm{NO}_{4}\left(\mathrm{M}^{+}+\mathrm{H}\right): 302.1387$, found 302.1387 .

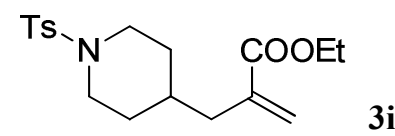

Ethyl 2-((1-tosylpiperidin-4-yl)methyl)acrylate (3i). White solid (46.4 mg, 66\%), mp: 53-55 ${ }^{\circ} \mathrm{C} ;{ }^{1} \mathrm{H}$ NMR $\left(400 \mathrm{MHz}, \mathrm{CDCl}_{3}\right): \delta 7.63(\mathrm{~d}, J=8.0 \mathrm{~Hz}, 2 \mathrm{H}), 7.31(\mathrm{~d}, J=$ $8.0 \mathrm{~Hz}, 2 \mathrm{H}), 6.15(\mathrm{~d}, J=1.2 \mathrm{~Hz}, 1 \mathrm{H}), 5.50(\mathrm{~s}, 1 \mathrm{H}), 4.15(\mathrm{q}, J=7.2 \mathrm{~Hz}, 2 \mathrm{H}), 3.77$ (brs, $1 \mathrm{H}), 3.74$ (brs, 1H), 2.43 (s, 3H), 2.22-2.16 (m, 4H), 1.72 (brs, 1H), 1.69 (brs, 1H), 1.48-1.18 (m, 6H); ${ }^{13} \mathrm{C}$ NMR $\left(100 \mathrm{MHz}, \mathrm{CDCl}_{3}\right): \delta 167.0,143.4,138.2,133.0,129.5$, $127.7,126.5,60.6,46.4,38.8,34.1,31.2,21.5,14.1$; IR (KBr): $v\left(\mathrm{~cm}^{-1}\right) 2982,2922$, 
2846, 1713, 1629, 1597, 1446, 1340, 1305, 1168, 1094, 931, 818, 727, 651, 549; ESI-MS: $(\mathrm{m} / \mathrm{z}) 352.2\left(\mathrm{M}^{+}+\mathrm{H}\right)$; HRMS calcd for $\mathrm{C}_{18} \mathrm{H}_{26} \mathrm{NO}_{4} \mathrm{~S}(\mathrm{M}+\mathrm{H}): 352.1577$, found 352.1574 .<smiles>C=C(CC1CCN(C(=O)c2ccccc2)CC1)C(=O)OCC</smiles>

Ethyl 2-((1-benzoylpiperidin-4-yl)methyl)acrylate (3j). Colorless oil (38.6 mg, 64\%); ${ }^{1} \mathrm{H}$ NMR (400 MHz, $\left.\mathrm{CDCl}_{3}\right): \delta 7.39$ (s, 5H), $6.20(\mathrm{~s}, 1 \mathrm{H}), 5.52(\mathrm{~s}, 1 \mathrm{H}), 4.70$ (brs, 1H), 4.21 (q, $J=7.2 \mathrm{~Hz}, 2 \mathrm{H}), 3.72$ (brs, 1H), 2.96 (brs, 1H), 2.74 (brs, 1H), 2.28 $(\mathrm{d}, J=6.8 \mathrm{~Hz}, 2 \mathrm{H}), 1.90-1.50(\mathrm{~m}, 3 \mathrm{H}), 1.32-1.06(\mathrm{~m}, 5 \mathrm{H}) ;{ }^{13} \mathrm{C}$ NMR (100 MHz, $\left.\mathrm{CDCl}_{3}\right): \delta 170.3,167.1,138.3,136.3,129.4,128.4,126.8,126.5,60.7,47.9,42.4$, 39.0, 35.2, 32.7, 31.7, 14.2; IR (neat): $v\left(\mathrm{~cm}^{-1}\right)$ 2981, 2932, 2855, 1713, 1631, 1577, 1446, 1370, 1285, 1161, 1090, 1073, 1026, 975, 788, 731, 709; ESI-MS: $(\mathrm{m} / \mathrm{z}) 302.2$ $\left(\mathrm{M}^{+}+\mathrm{H}\right)$; HRMS calcd for $\mathrm{C}_{18} \mathrm{H}_{24} \mathrm{NO}_{3}(\mathrm{M}+\mathrm{H})$ : 302.1751, found 302.1749 .

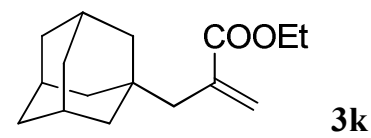

Ethyl 2-(adamantan-1-ylmethyl)acrylate (3k). Colorless oil (40.2 mg, 81\%); ${ }^{1} \mathrm{H}$ NMR (400 MHz, $\left.\mathrm{CDCl}_{3}\right): \delta 6.18(\mathrm{~d}, J=1.6 \mathrm{~Hz}, 1 \mathrm{H}), 5.40(\mathrm{~d}, J=0.4 \mathrm{~Hz}, 1 \mathrm{H}), 4.19$ (q, $J=7.2 \mathrm{~Hz}, 2 \mathrm{H}), 2.15(\mathrm{~s}, 2 \mathrm{H}), 1.93(\mathrm{~s}, 3 \mathrm{H}), 1.69-1.58(\mathrm{~m}, 6 \mathrm{H}), 1.45(\mathrm{~d}, J=2.0 \mathrm{~Hz}$, $6 \mathrm{H}), 1.30(\mathrm{t}, J=7.2 \mathrm{~Hz}, 3 \mathrm{H}) ;{ }^{13} \mathrm{C}$ NMR $\left(100 \mathrm{MHz} \mathrm{CDCl}_{3}\right): \delta 168.3,137.5,126.9$, 60.6, 45.4, 42.0, 36.9, 33.2, 28.6, 14.2; IR (neat): $v\left(\mathrm{~cm}^{-1}\right)$ 2980, 2904, 2847, 1720, 1625, 1449, 1311, 1297, 1208, 1179, 1133, 1028, 942; ESI-MS: $(m / z) 271.1\left(\mathrm{M}^{+}+\mathrm{Na}\right)$; HRMS calcd for $\mathrm{C}_{16} \mathrm{H}_{25} \mathrm{O}_{2}(\mathrm{M}+\mathrm{H}): 249.1849$, found 249.1848 .

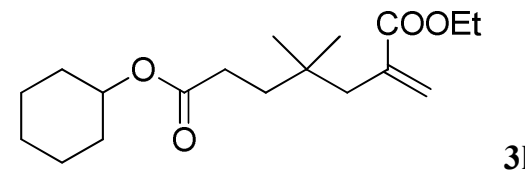

7-Cyclohexyl 1-ethyl 4,4-dimethyl-2-methyleneheptanedioate (31). Colorless oil (50.3 mg, 81\%); ${ }^{1} \mathrm{H}$ NMR (400 MHz, $\left.\mathrm{CDCl}_{3}\right): \delta 6.20(\mathrm{~d}, J=1.6 \mathrm{~Hz}, 1 \mathrm{H}), 5.47(\mathrm{~s}, 1 \mathrm{H})$, 4.79-4.70 (m, 1H), 4.19 (q, $J=7.2 \mathrm{~Hz}, 2 \mathrm{H}), 2.31-2.27(\mathrm{~m}, 4 \mathrm{H}), 1.90-1.68(\mathrm{~m}, 4 \mathrm{H})$, $1.58-1.53(\mathrm{~m}, 3 \mathrm{H}), 1.48-1.16(\mathrm{~m}, 8 \mathrm{H}), 0.84(\mathrm{~s}, 6 \mathrm{H}) ;{ }^{13} \mathrm{C} \mathrm{NMR}\left(100 \mathrm{MHz}, \mathrm{CDCl}_{3}\right): \delta$ 173.6, 168.1, 138.4, 127.4, 72.4, 60.7, 42.4, 36.6, 33.6, 31.6, 30.0, 26.1, 25.4, 23.7, 14.1; IR (neat): $v\left(\mathrm{~cm}^{-1}\right)$ 2938, 2860, 1721, 1626, 1468, 1451, 1388, 1367, 1302, 1259 , 1184, 1116, 1035, 1018, 947, 818; ESI-MS: $(\mathrm{m} / \mathrm{z}) 333.3\left(\mathrm{M}^{+}+\mathrm{Na}\right)$; HRMS calcd for $\mathrm{C}_{18} \mathrm{H}_{31} \mathrm{O}_{4}(\mathrm{M}+\mathrm{H}): 311.2217$, found 311.2216. 


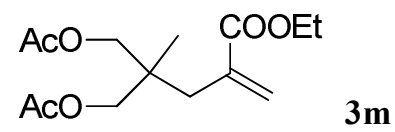

2-(2-(Ethoxycarbonyl)allyl)-2-methylpropane-1,3-diyl diacetate (3m). Colorless oil (34.4 mg, 60\%); ${ }^{1} \mathrm{H}$ NMR (400 MHz, $\left.\mathrm{CDCl}_{3}\right): \delta 6.27$ (d, $\left.J=1.2 \mathrm{~Hz}, 1 \mathrm{H}\right), 5.52$ (s, $1 \mathrm{H}), 4.19(\mathrm{q}, J=7.2 \mathrm{~Hz}, 2 \mathrm{H}), 3.92(\mathrm{~s}, 4 \mathrm{H}), 2.46(\mathrm{~s}, 2 \mathrm{H}), 2.06(\mathrm{~s}, 6 \mathrm{H}), 1.30(\mathrm{t}, J=7.2$ $\mathrm{Hz}, 3 \mathrm{H}), 0.91(\mathrm{~s}, 3 \mathrm{H}) ;{ }^{13} \mathrm{C} \mathrm{NMR}\left(100 \mathrm{MHz}, \mathrm{CDCl}_{3}\right): \delta 170.8,167.4,136.7,128.4$, 67.5, 60.9, 37.8, 35.7, 20.8, 19.0, 14.1; IR (neat): $v\left(\mathrm{~cm}^{-1}\right)$ 2979, 1742, 1718, 1627, 1469, 1378, 1230, 1180, 1124, 1040; EIMS: $m / z$ (rel intensity) $286\left(\mathrm{M}^{+}, 1\right), 244(43)$, 226 (12), 199 (45), 173 (21), 151 (44), 125 (44), 113 (26), 93 (30), 43 (100); HRMS calcd for $\mathrm{C}_{14} \mathrm{H}_{22} \mathrm{O}_{6}(\mathrm{M}): 286.1416$, found 286.1418 .

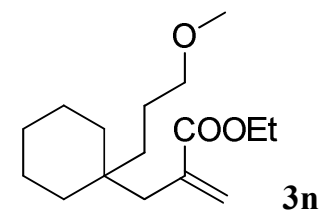

Ethyl 2-((1-(3-methoxypropyl)cyclohexyl)methyl)acrylate (3n). Colorless oil (41.9 mg, 78\%); ${ }^{1} \mathrm{H}$ NMR (400 MHz, $\left.\mathrm{CDCl}_{3}\right): \delta 6.16(\mathrm{~d}, J=2.0 \mathrm{~Hz}, 1 \mathrm{H}), 5.43(\mathrm{~s}, 1 \mathrm{H}), 4.19$ $(\mathrm{q}, J=7.2 \mathrm{~Hz}, 2 \mathrm{H}), 3.34-3.31(\mathrm{~m}, 5 \mathrm{H}), 2.33(\mathrm{~s}, 2 \mathrm{H}), 1.62-1.14(\mathrm{~m}, 17 \mathrm{H}) ;{ }^{13} \mathrm{C}$ NMR $\left(100 \mathrm{MHz}, \mathrm{CDCl}_{3}\right): \delta 168.5,138.6,127.0,73.7,60.6,58.5,39.4,36.0,34.8,31.6$, 26.1, 23.2, 21.6, 14.1; IR (neat): $v\left(\mathrm{~cm}^{-1}\right) 2927,2854,1721,1625,1459,1387,1368$, 1300, 1221, 1119, 1028, 950, 816; EIMS: $m / z$ (rel intensity) $269\left(\mathrm{M}^{+}+\mathrm{H}, 0.1\right), 236(1)$, 195 (3), 155 (74), 123 (100), 95 (16), 81 (75), 79 (13), 67 (24), 45 (12); HRMS calcd for $\mathrm{C}_{16} \mathrm{H}_{29} \mathrm{O}_{3}(\mathrm{M}+\mathrm{H}): 269.2117$, found 269.2111 .<smiles>C=C(CC(CC)(CC)CC)C(=O)OCC</smiles>

Ethyl 4-butyl-4-ethyl-2-methyleneoctanoate (3o). Colorless oil (40.3 mg, 75\%); ${ }^{1} \mathrm{H}$ NMR (400 MHz, $\left.\mathrm{CDCl}_{3}\right): \delta 6.11(\mathrm{~d}, J=1.6 \mathrm{~Hz}, 1 \mathrm{H}), 5.40(\mathrm{~s}, 1 \mathrm{H}), 4.18(\mathrm{q}, J=7.2 \mathrm{~Hz}$, $2 \mathrm{H}), 2.30(\mathrm{~s}, 2 \mathrm{H}), 1.31-1.08(\mathrm{~m}, 17 \mathrm{H}), 0.89(\mathrm{t}, J=6.8 \mathrm{~Hz}, 6 \mathrm{H}), 0.78(\mathrm{t}, J=7.2 \mathrm{~Hz}$, $3 \mathrm{H}) ;{ }^{13} \mathrm{C} \mathrm{NMR}\left(100 \mathrm{MHz}, \mathrm{CDCl}_{3}\right): \delta 168.6,139.3,126.2,60.6,38.8,36.6,35.5,28.3$, 25.3, 23.5, 14.2, 7.7; IR (neat): $v\left(\mathrm{~cm}^{-1}\right)$ 2958, 2931, 2862, 1721, 1625, 1464, 1159, 1127, 1029; ESI-MS: $(\mathrm{m} / \mathrm{z}) 269.3\left(\mathrm{M}^{+}+\mathrm{H}\right)$; HRMS calcd for $\mathrm{C}_{17} \mathrm{H}_{33} \mathrm{O}_{2}(\mathrm{M}+\mathrm{H})$ : 269.2475, found 269.2474.

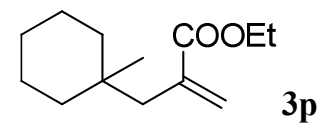

Ethyl 2-((1-methylcyclohexyl)methyl)acrylate (3p). Colorless oil (32.4 mg, 77\%); ${ }^{1} \mathrm{H}$ NMR $\left(400 \mathrm{MHz}, \mathrm{CDCl}_{3}\right): \delta 6.16(\mathrm{~d}, J=2.0 \mathrm{~Hz}, 1 \mathrm{H}), 5.43(\mathrm{~s}, 1 \mathrm{H}), 4.19(\mathrm{q}, J=7.2$ $\mathrm{Hz}, 2 \mathrm{H}), 2.31(\mathrm{~s}, 2 \mathrm{H}), 1.56-1.34(\mathrm{~m}, 5 \mathrm{H}), 1.30-1.23(\mathrm{~m}, 8 \mathrm{H}), 0.82(\mathrm{~s}, 3 \mathrm{H}) ;{ }^{13} \mathrm{C} \mathrm{NMR}$ $\left(100 \mathrm{MHz}, \mathrm{CDCl}_{3}\right): \delta 168.4,138.6,126.9,60.6,43.3,37.4,33.8,26.3,24.1,22.0$, 
14.1; IR (neat): $v\left(\mathrm{~cm}^{-1}\right)$ 2926, 2852, 1720, 1626, 1463, 1447, 1290, 1199, 1179, 1152 , 1028, 942, 852, 815; EIMS: $m / z$ (rel intensity) $210\left(\mathrm{M}^{+}, 4\right), 165$ (12), 150 (7), 114 (100), 97 (90), 86 (22), 69 (18), 55 (52); HRMS calcd for $\mathrm{C}_{13} \mathrm{H}_{22} \mathrm{O}_{2}$ (M): 210.1620, found 210.1617 .

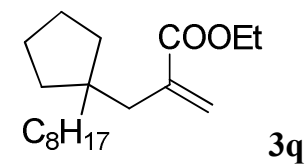

Ethyl 2-((1-octylcyclopentyl)methyl)acrylate (3q). Colorless oil $(48.3 \mathrm{mg}, 82 \%) ;{ }^{1} \mathrm{H}$ NMR (400 MHz, $\left.\mathrm{CDCl}_{3}\right): \delta 6.14(\mathrm{~d}, J=1.6 \mathrm{~Hz}, 1 \mathrm{H}), 5.45$ (s, 1H), 4.19 (q, $J=7.2 \mathrm{~Hz}$, $2 \mathrm{H}), 2.37(\mathrm{~s}, 2 \mathrm{H}), 1.67-1.57(\mathrm{~m}, 4 \mathrm{H}), 1.45-1.23(\mathrm{~m}, 21 \mathrm{H}), 0.88(\mathrm{t}, J=6.8 \mathrm{~Hz}, 3 \mathrm{H}) ;{ }^{13} \mathrm{C}$ NMR $\left(100 \mathrm{MHz}, \mathrm{CDCl}_{3}\right): \delta 168.5,139.5,126.4,60.6,45.9,38.4,38.2,36.4,31.9$, $30.5,29.6,29.4,24.5,24.0,22.6,14.1,14.0$; IR (neat): $v\left(\mathrm{~cm}^{-1}\right) 2926,2855,1721$, 1625, 1463, 1367, 1302, 1186, 1150, 1029, 941, 817; ESI-MS: $(\mathrm{m} / \mathrm{z}) 295.0\left(\mathrm{M}^{+}+\mathrm{H}\right)$; HRMS calcd for $\mathrm{C}_{19} \mathrm{H}_{35} \mathrm{O}_{2}(\mathrm{M}+\mathrm{H}): 295.2632$, found 295.2629.

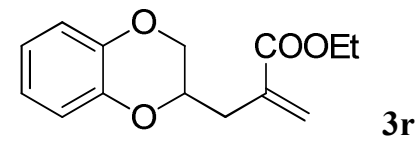

Ethyl 2-((2,3-dihydrobenzo[b] [1,4]dioxin-2-yl)methyl)acrylate (3r). Colorless oil (44.2 mg, 89\%); ${ }^{1} \mathrm{H}$ NMR (400 MHz, $\left.\mathrm{CDCl}_{3}\right): \delta 6.88-6.80(\mathrm{~m}, 4 \mathrm{H}), 6.33(\mathrm{~d}, J=0.8$ $\mathrm{Hz}, 1 \mathrm{H}), 5.72$ (d, $J=0.4 \mathrm{~Hz}, 1 \mathrm{H}), 4.39-4.34(\mathrm{~m}, 1 \mathrm{H}), 4.25-4.20(\mathrm{~m}, 3 \mathrm{H}), 3.92$ (dd, $J=$ 6.8, 11.2 Hz, 1H), $2.71(\mathrm{dd}, J=7.6,14.4 \mathrm{~Hz}, 1 \mathrm{H}), 2.64(\mathrm{dd}, J=5.6,14.4 \mathrm{~Hz}, 1 \mathrm{H})$, $1.31(\mathrm{t}, J=6.8 \mathrm{~Hz}, 3 \mathrm{H}) ;{ }^{13} \mathrm{C}$ NMR $\left(100 \mathrm{MHz}, \mathrm{CDCl}_{3}\right): \delta 166.7,143.1,142.9,135.4$, 128.4, 121.5, 121.3, 117.4, 117.0, 71.3, 67.3, 61.0, 33.7, 14.2; IR (neat): $v\left(\mathrm{~cm}^{-1}\right) 2981$, 2929, 1713, 1631, 1593, 1495, 1266, 1153, 1040, 748; ESI-MS: $(\mathrm{m} / \mathrm{z}) 249.1\left(\mathrm{M}^{+}+\mathrm{H}\right)$; HRMS calcd for $\mathrm{C}_{14} \mathrm{H}_{17} \mathrm{O}_{4}(\mathrm{M}+\mathrm{H})$ : 249.1121, found 249.1120.<smiles>C=C(CC(Cc1ccccc1)N1C(=O)CCC1=O)C(=O)OCC</smiles>

Ethyl 4-(2,5-dioxopyrrolidin-1-yl)-2-methylene-5-phenylpentanoate (3s). Colorless oil (49.2 mg, 78\%); ${ }^{1} \mathrm{H}$ NMR (400 MHz, $\mathrm{CDCl}_{3}$ ): $\delta$ 7.25-7.15 (m, 5H), 6.14 $(\mathrm{d}, J=1.2 \mathrm{~Hz}, 1 \mathrm{H}), 5.52(\mathrm{~s}, 1 \mathrm{H}), 4.72-4.64(\mathrm{~m}, 1 \mathrm{H}), 4.26-4.14(\mathrm{~m}, 2 \mathrm{H}), 3.28(\mathrm{dd}, J=$ 10.0, 13.6 Hz, 1H), 3.10-3.01 (m, 2H), 2.78 (dd, $J=4.0,14.0 \mathrm{~Hz}, 1 \mathrm{H}), 2.45$ (br, 4H), $1.30(\mathrm{t}, J=7.2 \mathrm{~Hz}, 3 \mathrm{H}) ;{ }^{13} \mathrm{C}$ NMR $\left(100 \mathrm{MHz}, \mathrm{CDCl}_{3}\right): \delta 177.3,166.3,137.7,137.5$, $128.9,128.4,127.3,126.6,60.9,52.3,37.4,34.0,27.7,14.1$; IR (neat): $v\left(\mathrm{~cm}^{-1}\right) 1774$, 1703, 1630, 1396, 1370, 1191, 1025, 749, 702; ESI-MS: $(\mathrm{m} / \mathrm{z}) 316.1\left(\mathrm{M}^{+}+\mathrm{H}\right)$; HRMS calcd for $\mathrm{C}_{18} \mathrm{H}_{22} \mathrm{NO}_{4}(\mathrm{M}+\mathrm{H}): 316.1543$, found 316.1543 . 


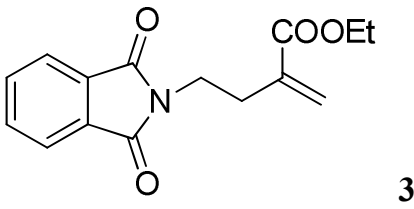

Ethyl 4-(1,3-dioxoisoindolin-2-yl)-2-methylenebutanoate (3t). Colorless oil (25.1 mg, 46\%); ${ }^{1} \mathrm{H}$ NMR (400 MHz, $\left.\mathrm{CDCl}_{3}\right): \delta$ 7.83-7.81 (m, 2H), 7.71-7.68 (m, 2H), 6.14 (s, 1H), 5.51 (s, 1H), 4.23 (q, $J=7.2 \mathrm{~Hz}, 2 \mathrm{H}), 3.89$ (t, $J=6.8 \mathrm{~Hz}, 2 \mathrm{H}), 2.69$ (t, $J=6.8$ $\mathrm{Hz}, 2 \mathrm{H}), 1.31(\mathrm{t}, J=7.2 \mathrm{~Hz}, 3 \mathrm{H}) ;{ }^{13} \mathrm{C} \mathrm{NMR}\left(100 \mathrm{MHz}, \mathrm{CDCl}_{3}\right): \delta 168.2,166.4,137.4$, 133.9, 132.1, 127.1, 123.2, 60.9, 36.9, 31.4, 14.2; IR (neat): $v\left(\mathrm{~cm}^{-1}\right)$ 2982, 2940, 1773, 1713, 1631, 1467, 1440, 1395, 1367, 1179, 1027; ESI-MS: $(\mathrm{m} / \mathrm{z}) 296.2\left(\mathrm{M}^{+}+\mathrm{Na}\right)$, $274.0\left(\mathrm{M}^{+}+\mathrm{H}\right)$; HRMS calcd for $\mathrm{C}_{15} \mathrm{H}_{16} \mathrm{O}_{4} \mathrm{~N}(\mathrm{M}+\mathrm{H})$ : 274.1074, found 274.1070.<smiles>C=C(CCCCCCN1C(=O)c2ccccc2C1=O)C(=O)OCC</smiles>

Ethyl 8-(1,3-dioxoisoindolin-2-yl)-2-methyleneoctanoate (3u). Colorless oil (30.3 mg, 46\%); ${ }^{1} \mathrm{H}$ NMR (400 MHz, $\left.\mathrm{CDCl}_{3}\right): \delta$ 7.86-7.81 (m, 2H), 7.73-7.68 (m, 2H), 6.11 $(\mathrm{s}, 1 \mathrm{H}), 5.49(\mathrm{~d}, J=1.2 \mathrm{~Hz}, 1 \mathrm{H}), 4.19(\mathrm{q}, J=7.2 \mathrm{~Hz}, 2 \mathrm{H}), 3.67(\mathrm{t}, J=7.2 \mathrm{~Hz}, 2 \mathrm{H})$, $2.27(\mathrm{t}, J=7.2 \mathrm{~Hz}, 2 \mathrm{H}), 1.70-1.63(\mathrm{~m}, 2 \mathrm{H}), 1.49-1.33(\mathrm{~m}, 6 \mathrm{H}), 1.29(\mathrm{t}, J=7.2 \mathrm{~Hz}$, $3 \mathrm{H}) ;{ }^{13} \mathrm{C} \mathrm{NMR}\left(100 \mathrm{MHz}, \mathrm{CDCl}_{3}\right): \delta 168.5,167.4,141.0,133.9,132.2,124.3,123.2$, 60.5, 38.0, 31.7, 28.8, 28.5, 28.2, 26.7, 14.2; IR (neat): $v\left(\mathrm{~cm}^{-1}\right)$ 2934, 2859, 1773, 1713, 1631, 1467, 1438, 1396, 1369, 1186, 1052, 913, 742, 719; ESI-MS: $(\mathrm{m} / \mathrm{z}) 352.1$ $\left(\mathrm{M}^{+}+\mathrm{Na}\right)$, $330.1\left(\mathrm{M}^{+}+\mathrm{H}\right)$; HRMS calcd for $\mathrm{C}_{19} \mathrm{H}_{24} \mathrm{NO}_{4}(\mathrm{M}+\mathrm{H})$ : 330.1700, found 330.1696 .<smiles>C=C(C[C@@H]1CCCC[C@H]1C(=O)c1ccccc1)C(=O)OCC</smiles>

Ethyl 2-(((1R*,2R*)-2-benzoylcyclohexyl)methyl)acrylate (cis-3v). Colorless oil (6.3 mg, 10\% from trans-1v; $6.6 \mathrm{mg}, 11 \%$ from cis-1v); ${ }^{1} \mathrm{H}$ NMR (400 MHz, $\left.\mathrm{CDCl}_{3}\right)$ : $\delta$ 7.92-7.89 (m, 2H), 7.55-7.51 (m, 1H), 7.47-7.43 (m, 2H), $6.06(\mathrm{~d}, J=1.6 \mathrm{~Hz}, 1 \mathrm{H})$, $5.32(\mathrm{~s}, 1 \mathrm{H}), 4.09-3.90(\mathrm{~m}, 2 \mathrm{H}), 3.60-3.56(\mathrm{~m}, 1 \mathrm{H}), 2.40(\mathrm{dd}, J=8.8,13.6 \mathrm{~Hz}, 1 \mathrm{H})$, $2.25(\mathrm{dd}, J=5.6,14.0 \mathrm{~Hz}, 1 \mathrm{H}), 2.19-2.11(\mathrm{~m}, 1 \mathrm{H}), 1.96-1.81(\mathrm{~m}, 2 \mathrm{H}), 1.72-1.49(\mathrm{~m}$, $4 \mathrm{H}), 1.42-1.34(\mathrm{~m}, 2 \mathrm{H}), 1.10(\mathrm{t}, J=7.2 \mathrm{~Hz}, 3 \mathrm{H}) ;{ }^{13} \mathrm{C} \mathrm{NMR}\left(100 \mathrm{MHz}, \mathrm{CDCl}_{3}\right): \delta$ 203.3, 167.1, 139.2, 137.1, 132.6, 128.5, 128.1, 126.6, 60.4, 46.3, 36.8, 32.8, 28.2, 25.5, 23.6, 22.8, 14.0; IR (neat): $v\left(\mathrm{~cm}^{-1}\right)$ 2927, 2855, 1716, 1679, 1629, 1596, 1448, 1301, 1255, 1190, 1177, 1148, 1026, 940, 705; ESI-MS: $(\mathrm{m} / \mathrm{z}) 301.1\left(\mathrm{M}^{+}+\mathrm{H}\right)$; HRMS calcd for $\mathrm{C}_{19} \mathrm{H}_{25} \mathrm{O}_{3}(\mathrm{M}+\mathrm{H}): 301.1798$, found 301.1795 . 


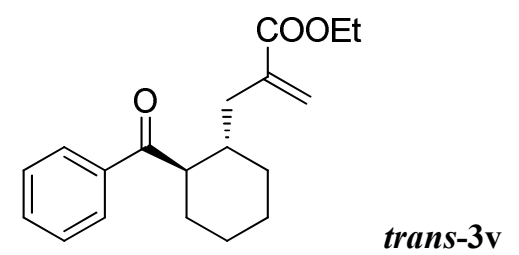

Ethyl 2-(((1S*,2R*)-2-benzoylcyclohexyl)methyl)acrylate (trans-3v). Colorless oil (35.8 mg, 60\% from trans-1v; $37.3 \mathrm{mg}, 62 \%$ from cis-1v); ${ }^{1} \mathrm{H}$ NMR (400 MHz, $\left.\mathrm{CDCl}_{3}\right): \delta 7.97-7.95(\mathrm{~m}, 2 \mathrm{H}), 7.57-7.53(\mathrm{~m}, 1 \mathrm{H}), 7.48-7.44(\mathrm{~m}, 2 \mathrm{H}), 6.10(\mathrm{~d}, J=0.8$ $\mathrm{Hz}, 1 \mathrm{H}), 5.42(\mathrm{~s}, 1 \mathrm{H}), 4.24-4.08(\mathrm{~m}, 2 \mathrm{H}), 3.18-3.12(\mathrm{~m}, 1 \mathrm{H}), 2.32-2.29(\mathrm{~m}, 1 \mathrm{H})$, 2.22-2.07 (m, 2H), 1.91-1.70 (m, 4H), 1.45-1.23 (m, 6H), $1.08-0.96(\mathrm{~m}, 1 \mathrm{H}) ;{ }^{13} \mathrm{C}$ NMR (100 MHz, $\left.\mathrm{CDCl}_{3}\right): \delta 203.8,167.3,139.1,137.3,132.8,128.6,128.1,126.1$, $60.6,50.5,38.3,37.1,31.1,30.9,25.9,25.6,14.1$; IR (neat): $v\left(\mathrm{~cm}^{-1}\right) 2930,2855$, 1717, 1681, 1447, 1368, 1302, 1261, 1195, 1150, 1098, 702; ESI-MS: $(\mathrm{m} / \mathrm{z}) 301.1$ $\left(\mathrm{M}^{+}+\mathrm{H}\right)$; HRMS calcd for $\mathrm{C}_{19} \mathrm{H}_{25} \mathrm{O}_{3}(\mathrm{M}+\mathrm{H}): 301.1798$, found 301.1794 .

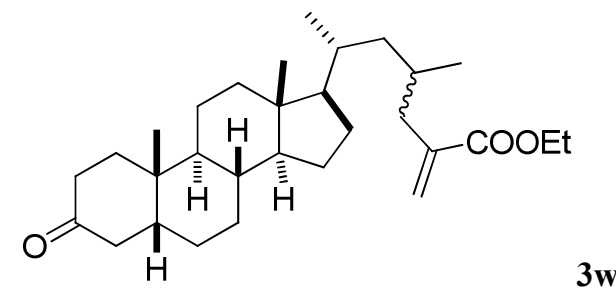

Compound 3w. This compound was isolated as the mixture of two stereoisomers in approximately 1:1 ratio determined by ${ }^{13} \mathrm{C}$ NMR. Yield: $73.1 \mathrm{mg}$ (80\%). Colorless oil; ${ }^{1} \mathrm{H}$ NMR $\left(400 \mathrm{MHz}, \mathrm{CDCl}_{3}\right.$ ): $\delta 6.18$ (brs, $\left.1 \mathrm{H}\right), 5.47$ (brs, $\left.1 \mathrm{H}\right), 4.26-4.14(\mathrm{~m}, 2 \mathrm{H})$, 2.74-1.17 (m, 27H), 1.15-0.84 (m, 11H), $0.81(\mathrm{~d}, J=6.4 \mathrm{~Hz}, 3 \mathrm{H}), 0.70(\mathrm{~d}, J=4.0 \mathrm{~Hz}$, $3 \mathrm{H}) ;{ }^{13} \mathrm{C}$ NMR $\left(100 \mathrm{MHz}, \mathrm{CDCl}_{3}\right): \delta 213.4,167.5,167.4,140.0,139.8,125.7,125.5$, $60.5,57.3,57.1,56.5,56.5,44.6,44.3,43.2,42.8,42.3,41.1,40.7,40.1,38.7,37.2$, $37.0,35.5,34.9,33.7,33.6,29.0,28.8,28.5,26.6,25.7,24.2,22.6,21.2,20.9,18.9$, 18.8, 18.4, 14.2, 12.1; IR (neat): $v\left(\mathrm{~cm}^{-1}\right)$ 2934, 2866, 1716, 1629, 1446, 1378, 1324, 1299, 1267, 1200, 1159, 1097, 1027, 943, 820, 736; ESI-MS: $(m / z) 479.4\left(\mathrm{M}^{+}+\mathrm{Na}\right)$; HRMS calcd for $\mathrm{C}_{30} \mathrm{H}_{49} \mathrm{O}_{3}(\mathrm{M}+\mathrm{H})$ : 457.3676, found 457.3668.<smiles>C=C(CC(CC)Cc1ccc(C(=O)O)cc1)OCC</smiles>

4-(4-(Ethoxycarbonyl)-2-ethylpent-4-en-1-yl)benzoic acid (5). White solid (31.4 mg, 54\%), mp: $34-37{ }^{\circ} \mathrm{C} ;{ }^{1} \mathrm{H}$ NMR $\left(400 \mathrm{MHz}, \mathrm{CDCl}_{3}\right): \delta 8.02(\mathrm{~d}, J=8.0 \mathrm{~Hz}, 2 \mathrm{H})$, $7.25(\mathrm{~d}, J=7.6 \mathrm{~Hz}, 2 \mathrm{H}), 6.21(\mathrm{~d}, J=1.6 \mathrm{~Hz}, 1 \mathrm{H}), 5.52$ (d, $J=1.2 \mathrm{~Hz}, 1 \mathrm{H}), 4.19$ (q, $J$ $=7.2 \mathrm{~Hz}, 2 \mathrm{H}), 2.67-2.57(\mathrm{~m}, 2 \mathrm{H}), 2.36-2.20(\mathrm{~m}, 2 \mathrm{H}), 1.95-1.85(\mathrm{~m}, 1 \mathrm{H}), 1.33-1.22(\mathrm{~m}$, $5 \mathrm{H}), 0.90(\mathrm{t}, J=7.2 \mathrm{~Hz}, 3 \mathrm{H}) ;{ }^{13} \mathrm{C} \mathrm{NMR}\left(100 \mathrm{MHz}, \mathrm{CDCl}_{3}\right): \delta 171.8,167.3,147.9$, 139.5, 130.1, 129.3, 126.9, 126.3, 60.7, 39.9, 39.8, 36.2, 25.2, 14.2, 10.6; IR (KBr): $v$ $\left(\mathrm{cm}^{-1}\right)$ 2963, 2929, 1716, 1690, 1629, 1610, 1575, 1422, 1289, 1181, 1148, 1021, 943, 861, 807; EIMS: $m / z$ (rel intensity) $290\left(\mathrm{M}^{+}, 72\right), 244$ (44), 227 (16), 215 (18), 177 
(100), 159 (35), 135 (71), 109 (37), 91 (37); HRMS calcd for $\mathrm{C}_{17} \mathrm{H}_{22} \mathrm{O}_{4}$ (M): 290.1518, found 290.1515 .

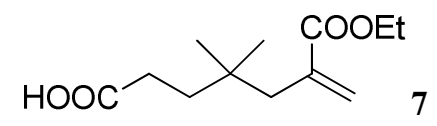

6-(Ethoxycarbonyl)-4,4-dimethylhept-6-enoic acid (7). Colorless oil (34.6 mg, $60 \%) ;{ }^{1} \mathrm{H}$ NMR $\left(400 \mathrm{MHz}, \mathrm{CDCl}_{3}\right): \delta 6.22(\mathrm{~d}, J=1.6 \mathrm{~Hz}, 1 \mathrm{H}), 5.48(\mathrm{~d}, J=0.8 \mathrm{~Hz}$, $1 \mathrm{H}), 4.20$ (q, $J=7.2 \mathrm{~Hz}, 2 \mathrm{H}), 2.39-2.35(\mathrm{~m}, 2 \mathrm{H}), 2.30(\mathrm{~s}, 2 \mathrm{H}), 1.59-1.55(\mathrm{~m}, 2 \mathrm{H})$, $1.30(\mathrm{t}, J=7.2 \mathrm{~Hz}, 3 \mathrm{H}), 0.85(\mathrm{~s}, 6 \mathrm{H}) ;{ }^{13} \mathrm{C} \mathrm{NMR}\left(100 \mathrm{MHz}, \mathrm{CDCl}_{3}\right): \delta 179.6,168.1$, 138.2, 127.6, 60.8, 42.3, 36.2, 33.6, 29.3, 26.1, 14.1; IR (neat): $v\left(\mathrm{~cm}^{-1}\right)$ 2962, 1713, 1626, 1413, 1305, 1262, 1184, 1115, 1025, 947, 803; EIMS: $m / z$ (rel intensity) 213 $\left(\mathrm{M}^{+}-\mathrm{CH}_{3}, 0.72\right), 194$ (8), 183 (13), 149 (14), 115 (100), 114 (41), 109 (14), 97 (59), 86 (27), 69 (47); HRMS calcd for $\mathrm{C}_{11} \mathrm{H}_{17} \mathrm{O}_{4}\left(\mathrm{M}-\mathrm{CH}_{3}\right): 213.1127$, found 213.1122.<smiles>CCCC(=S)CC(CC)CCC</smiles>

4-Ethyl-2-methylenehexadecanenitrile (8). Colorless oil (40.0 mg, 72\%); ${ }^{1} \mathrm{H}$ NMR $\left(400 \mathrm{MHz}, \mathrm{CDCl}_{3}\right): \delta 5.87(\mathrm{~s}, 1 \mathrm{H}), 5.68(\mathrm{~d}, J=0.8 \mathrm{~Hz}, 1 \mathrm{H}), 2.18(\mathrm{~d}, J=6.8 \mathrm{~Hz}, 2 \mathrm{H})$, $1.63-1.54(\mathrm{~m}, 1 \mathrm{H}), 1.40-1.26(\mathrm{~m}, 24 \mathrm{H}), 0.90-0.85(\mathrm{~m}, 6 \mathrm{H}) ;{ }^{13} \mathrm{C}$ NMR $(100 \mathrm{MHz}$, $\left.\mathrm{CDCl}_{3}\right): \delta 131.2,122.7,118.9 .38 .9,37.5,32.3,31.9,29.9,29.6,29.3,26.3,25.1,22.7$, 14.1, 10.4; IR (neat): $v\left(\mathrm{~cm}^{-1}\right)$ 2925, 2854, 1463, 1380, 928, 721; EIMS: $m / z$ (rel intensity) 277 ( $\left.\mathrm{M}^{+}, 15\right), 262$ (18), 248 (99), 206 (65), 192 (87), 178 (63), 164 (46), 150 (44), 108 (51), 85 (82), 71 (100), 57 (99), 43 (70); HRMS calcd for $\mathrm{C}_{19} \mathrm{H}_{35} \mathrm{~N}(\mathrm{M})$ : 277.2770, found 277.2773.

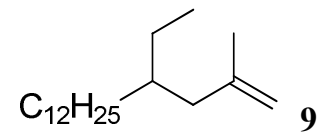

4-Ethyl-2-methylhexadec-1-ene (9). Colorless oil (23.5 mg, 44\%); ${ }^{1} \mathrm{H}$ NMR (400 $\left.\mathrm{MHz}, \mathrm{CDCl}_{3}\right): \delta 4.73(\mathrm{~s}, 1 \mathrm{H}), 4.65(\mathrm{~s}, 1 \mathrm{H}), 1.93(\mathrm{~d}, J=6.8 \mathrm{~Hz}, 2 \mathrm{H}), 1.68(\mathrm{~s}, 3 \mathrm{H})$, 1.49-1.37 (m, 1H), 1.36-1.16 (m, 24H), 0.90-0.83 (m, 6H); ${ }^{13} \mathrm{C}$ NMR (100 MHz, $\left.\mathrm{CDCl}_{3}\right): \delta 145.2,111.2,42.6,36.5,32.8,31.9,30.1,29.7,29.3,26.5,25.6,22.7,22.3$, 14.1, 10.7; IR (neat): $v\left(\mathrm{~cm}^{-1}\right)$ 3073, 2959, 2924, 2854, 1458, 1377, 887, 721; EIMS: $\mathrm{m} / \mathrm{z}$ (rel intensity) $266\left(\mathrm{M}^{+}, 11\right), 237$ (14), 210 (100), 182 (10), 153 (8), 125 (21), 111 (35), 97 (62), 69 (41), 57 (50), 43 (35); HRMS calcd for $\mathrm{C}_{19} \mathrm{H}_{38}$ (M): 266.2974, found 266.2979 .<smiles>C=CCC(CC)CC</smiles>

4-Ethylhexadec-1-ene (10). Colorless oil (25.2 mg, 50\%); ${ }^{1} \mathrm{H}$ NMR (400 MHz, $\left.\mathrm{CDCl}_{3}\right): \delta 5.82-5.72(\mathrm{~m}, 1 \mathrm{H}), 5.02-4.95(\mathrm{~m}, 2 \mathrm{H}), 2.03-2.00(\mathrm{~m}, 2 \mathrm{H}), 1.31-1.21(\mathrm{~m}$, 
25H), 0.90-0.83 (m, 6H); ${ }^{13} \mathrm{C}$ NMR (100 MHz, $\left.\mathrm{CDCl}_{3}\right): \delta 137.7,115.3,38.9,37.7$, 32.9, 31.9, 30.0, 29.7, 29.3, 26.7, 25.7, 22.7, 14.1, 10.9; IR (neat): $v\left(\mathrm{~cm}^{-1}\right) 3075,2959$, 2929, 2854, 1464, 1378, 994, 909; EIMS: $m / z$ (rel intensity) $252\left(\mathrm{M}^{+}, 11\right), 210$ (77), 182 (11), 155 (15), 141 (20), 127 (24), 113 (25), 99 (38), 85 (87), 71 (96), 57 (100), 43 (67); HRMS calcd for $\mathrm{C}_{18} \mathrm{H}_{36}(\mathrm{M}): 252.2817$, found 252.2823 .<smiles>C=C(C#N)CC1COc2ccccc2O1</smiles>

2-((2,3-Dihydrobenzo[b] [1,4]dioxin-2-yl)methyl)acrylonitrile (11). Colorless oil (22.5 mg, 56\%); ${ }^{1} \mathrm{H}$ NMR (400 MHz, $\left.\mathrm{CDCl}_{3}\right): \delta 6.90-6.83(\mathrm{~m}, 4 \mathrm{H}), 6.04(\mathrm{~s}, 1 \mathrm{H}), 5.88$ (s, 1H), 4.45-4.40 (m, 1H), $4.26(\mathrm{dd}, J=2.4,11.2 \mathrm{~Hz}, 1 \mathrm{H}), 3.97$ (dd, $J=6.4,11.2 \mathrm{~Hz}$, $1 \mathrm{H}), 2.67$ (dd, $J=8.4,14.8 \mathrm{~Hz}, 1 \mathrm{H}), 2.55(\mathrm{dd}, J=5.2,14.8 \mathrm{~Hz}, 1 \mathrm{H}) ;{ }^{13} \mathrm{C}$ NMR $(100$ $\left.\mathrm{MHz}, \mathrm{CDCl}_{3}\right): \delta 142.8,142.3,133.9,121.9,121.7,118.1,118.0,117.5,117.2,70.3$, 66.6, 36.0; IR (neat): $v\left(\mathrm{~cm}^{-1}\right)$ 3042, 2925, 2882, 2224, 1593, 1494, 1260, 1200, 1120 , 1098, 1076, 1042, 947, 838, 752; ESI-MS: $(\mathrm{m} / \mathrm{z}) 224.1\left(\mathrm{M}^{+}+\mathrm{Na}\right)$; HRMS calcd for $\mathrm{C}_{12} \mathrm{H}_{12} \mathrm{NO}_{2}(\mathrm{M}+\mathrm{H}): 202.0863$, found 202.0862.

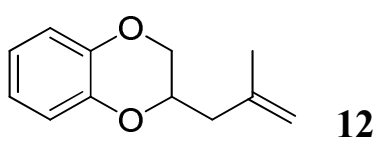

2-(2-Methylallyl)-2,3-dihydrobenzo[b][1,4]dioxine (12). Colorless oil (28.9 mg, $76 \%$ ); ${ }^{1} \mathrm{H}$ NMR (400 MHz, $\left.\mathrm{CDCl}_{3}\right): \delta 6.88-6.81(\mathrm{~m}, 4 \mathrm{H}), 4.90(\mathrm{~s}, 1 \mathrm{H}), 4.83(\mathrm{~s}, 1 \mathrm{H})$, $4.33-4.22(\mathrm{~m}, 2 \mathrm{H}), 3.88(\mathrm{dd}, J=7.6,11.6 \mathrm{~Hz}, 1 \mathrm{H}), 2.48(\mathrm{dd}, J=6.8,14.4 \mathrm{~Hz}, 1 \mathrm{H})$, $2.30(\mathrm{dd}, J=6.8,14.4 \mathrm{~Hz}, 1 \mathrm{H}), 1.83(\mathrm{~s}, 3 \mathrm{H}) ;{ }^{13} \mathrm{C} \mathrm{NMR}\left(100 \mathrm{MHz}, \mathrm{CDCl}_{3}\right): \delta 143.3$, 143.2, 140.6, 121.5, 121.2, 117.4, 117.0, 113.8, 71.3, 67.6, 39.3, 22.7; IR (neat): $v$ $\left(\mathrm{cm}^{-1}\right)$ 3076, 2971, 2933, 1651, 1593, 1495, 1465, 1261, 1079, 1044, 896, 748; EIMS: m/z (rel intensity) $190\left(\mathrm{M}^{+}, 100\right), 135$ (95), 134 (28), 121 (14), 107 (18), 81 (29), 79 (23), 77 (12); HRMS calcd for $\mathrm{C}_{12} \mathrm{H}_{14} \mathrm{O}_{2}$ (M): 190.0994, found 190.0991 .

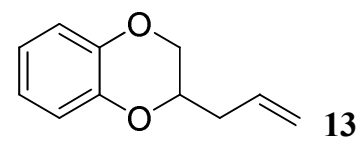

2-Allyl-2,3-dihydrobenzo[b][1,4]dioxine (13). Colorless oil (19.4 mg, 55\%); ${ }^{1} \mathrm{H}$ NMR (400 MHz, $\left.\mathrm{CDCl}_{3}\right): \delta$ 6.89-6.81 (m, 4H), 5.94-5.83 (m, 1H), 5.21-5.15 (m, 2H), 4.25-4.15 (m, 2H), $3.90(\mathrm{dd}, J=7.6,11.2 \mathrm{~Hz}, 1 \mathrm{H}), 2.56-2.49(\mathrm{~m}, 1 \mathrm{H}), 2.42-2.35(\mathrm{~m}$, $1 \mathrm{H}) ;{ }^{13} \mathrm{C}$ NMR $\left(100 \mathrm{MHz}, \mathrm{CDCl}_{3}\right): \delta 143.3,143.2,132.5,121.5,121.2,118.4,117.3$, 117.0, 72.3, 67.4, 35.5; IR (neat): $v\left(\mathrm{~cm}^{-1}\right)$ 3078, 2925, 1643, 1593, 1495, 1267, 1199, 1045, 993, 916, 747; EIMS: $m / z$ (rel intensity) $176\left(\mathrm{M}^{+}, 79\right), 135$ (100), 121 (14), 107 (26), 91 (7), 81 (12), 77 (14), 67 (17), 52 (13); HRMS calcd for $\mathrm{C}_{11} \mathrm{H}_{12} \mathrm{O}_{2}$ (M): 176.0837, found 176.0842 . 


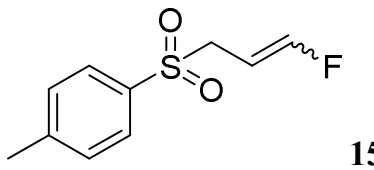

1-((3-Fluoroallyl)sulfonyl)-4-methylbenzene (15). This compound was isolated as the mixture of $Z$ - and $E$-isomers in 62:38 ratio determined by ${ }^{1} \mathrm{H}$ NMR. Yield: 30.0 mg (70\%). Colorless oil; ${ }^{1} \mathrm{H}$ NMR (400 MHz, $\left.\mathrm{CDCl}_{3}\right): \delta$ 7.79-7.74 (m, 2H), 7.38-7.34 $(\mathrm{m}, 2 \mathrm{H}), 6.57(\mathrm{dd}, J=82.4,4.4 \mathrm{~Hz}, 0.62 \mathrm{H}), 6.48(\mathrm{dd}, J=80.8,11.2 \mathrm{~Hz}, 0.38 \mathrm{H})$, $5.42-5.31(\mathrm{~m}, 0.38 \mathrm{H}), 5.05-4.90(\mathrm{M}, 0.62 \mathrm{H}), 3.89(\mathrm{~d}, J=8.0 \mathrm{~Hz}, 1.24 \mathrm{H}), 3.64(\mathrm{~d}, J=$ $8.4 \mathrm{~Hz}, 0.76 \mathrm{H}), 2.46 / 2.45(2 \mathrm{~s}, 3 \mathrm{H}) ;{ }^{13} \mathrm{C} \mathrm{NMR}\left(100 \mathrm{MHz}, \mathrm{CDCl}_{3}\right): \delta 154.6(\mathrm{~d}, J=$ $264.8 \mathrm{~Hz}) / 152.4(\mathrm{~d}, J=266.2 \mathrm{~Hz}), \quad 145.1 / 144.9, \quad 135.3 / 134.9,129.9 / 129.8$, 128.5/128.4, $100.4(\mathrm{~d}, J=16.8 \mathrm{~Hz}) / 99.0(\mathrm{~d}, J=3.7 \mathrm{~Hz}), 53.7(\mathrm{~d}, J=10.9 \mathrm{~Hz}) / 51.3(\mathrm{~d}$, $J=5.1 \mathrm{~Hz}), 21.6 ;{ }^{19} \mathrm{~F}$ NMR $\left(376 \mathrm{MHz}, \mathrm{CDCl}_{3}\right): \delta-116.0(\mathrm{dd}, J=79.3,14.7 \mathrm{~Hz}$, $0.36 \mathrm{~F}),-121.9(\mathrm{dd}, J=38.4,81.2 \mathrm{~Hz}, 0.64 \mathrm{~F})$; EIMS: $m / z$ (rel intensity) $214\left(\mathrm{M}^{+}, 2\right)$, 184 (4), 166 (5), 155 (85), 149 (26), 139 (15), 118 (10), 91 (100), 65 (22), 59 (36); HRMS calcd for $\mathrm{C}_{10} \mathrm{H}_{11} \mathrm{FO}_{2} \mathrm{~S}(\mathrm{M}): 214.0464$, found 214.0463 .

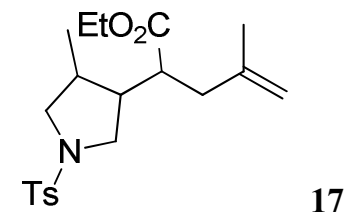

Ethyl 4-methyl-2-(4-methyl-1-tosylpyrrolidin-3-yl)pent-4-enoate (17). Colorless oil (34.9 mg, 46\%); ${ }^{1} \mathrm{H}$ NMR (400 MHz, $\left.\mathrm{CDCl}_{3}\right): \delta 7.72-7.68(\mathrm{~m}, 2 \mathrm{H}), 7.36-7.32(\mathrm{~m}$, $2 \mathrm{H})$, 4.72-4.64 (m, 2H), 4.13-3.99 (m, 2H), 3.52-2.73 (m, 5H), 2.55-1.75 (m, 7H), $1.70 / 1.66(2 \mathrm{~s}, 3 \mathrm{H}), 1.27-1.16(\mathrm{~m}, 3 \mathrm{H}), 0.95 / 0.85 / 0.75 / 0.68(4 \mathrm{~d}, J=6.8 \mathrm{~Hz}, 3 \mathrm{H}) ;{ }^{13} \mathrm{C}$ NMR $\left(100 \mathrm{MHz}, \mathrm{CDCl}_{3}\right): \delta 173.9,173.2,143.5,143.5,143.4,142.2,142.2,142.1$, 134.1, 133.6, 133.4, 129.7, 129.6, 127.6, 127.6, 127.4, 112.8, 112.5, 112.5, 60.6, 60.4. $60.4,55.5,55.3,54.8,51.1,50.0,48.8,47.4,47.2,46.4,44.5,44.2,44.1,44.0,43.6$, $40.5,39.0,38.8,38.6,36.9,36.2,34.5,33.4,31.9,29.7,29.4,22.7,22.2,22.2,22.1$, 21.5, 17.4, 16.8, 15.3, 14.2, 14.2, 14.1, 13.3, 12.9; IR (neat): $v\left(\mathrm{~cm}^{-1}\right) 2925,1729$, 1597, 1345, 1289, 1270, 1246, 1163, 1093, 1037, 913, 815, 742; ESI-MS: $(\mathrm{m} / \mathrm{z}) 402.1$ $\left(\mathrm{M}^{+}+\mathrm{Na}\right), 380.2\left(\mathrm{M}^{+}+\mathrm{H}\right)$; HRMS calcd for $\mathrm{C}_{20} \mathrm{H}_{30} \mathrm{NO}_{4} \mathrm{~S}(\mathrm{M}+\mathrm{H})$ : 380.1890 , found 380.1887 . 
4. References for Known Compounds.

\begin{tabular}{|c|c|c|}
\hline No. & reference & compd \\
\hline 1 & $\begin{array}{c}\text { Wang, Z.; Zhu, L.; Yin, F.; Su, Z.; Li, Z.; Li ,C. J. Am. } \\
\text { Chem. Soc. 2012, 134, } 4258 .\end{array}$ & $\begin{array}{l}\text { 1a, 1b, 1d, 1e, 1j, } 1 \text {, } \\
\text { 1q, 1s, 1t, cis-1v, } \\
\text { trans-1v, } 14\end{array}$ \\
\hline 2 & 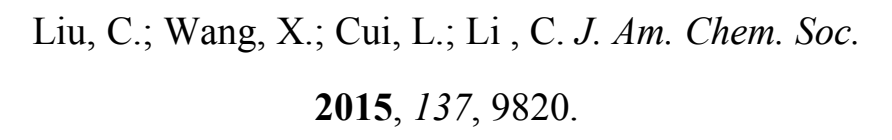 & $\begin{array}{l}\text { 1c, } 1 \mathrm{~g}, 1 \mathrm{i}, 1 \mathrm{k}, 1 \mathrm{~m} \\
1 \mathrm{o}, 1 \mathrm{r}, 1 \mathrm{w}, 4,6\end{array}$ \\
\hline 3 & $\begin{array}{l}\text { Liu, X.; Wang, Z.; Cheng, X.; Li, C. J. Am. Chem. Soc. } \\
\text { 2012, 134, } 14330 .\end{array}$ & $1 \mathrm{f}, 1 \mathrm{n}, 1 \mathrm{p}$ \\
\hline 4 & $\begin{array}{c}\text { Yin, F.; Wang, Z.; Zhu, L.; Li, Z.; Li, C.J. Am. Chem. } \\
\text { Soc. 2012, 134, } 10401\end{array}$ & 1u \\
\hline 5 & $\begin{array}{l}\text { Corc, V.; Chamoreau, L.; Derat, E.; Goddard, J.; } \\
\text { Ollivier, C.; Fensterbank, L. Angew. Chem. 2015, } \\
\text { 127, } 11576\end{array}$ & $2 a$ \\
\hline 6 & $\begin{array}{l}\text { Harvey, I.; Phillips, E.; Whitham, G. } \\
\text { Tetrahedron 1997, 53, } 6493\end{array}$ & $2 b$ \\
\hline 7 & $\begin{array}{l}\text { Larraufie, M.; Pellet, R.; Fensterbank, L.; Goddard, J.; } \\
\text { Lacote, E.; Malacria, M.; Ollivier, C. Angew. Chem., } \\
\text { Int. Ed. 2011, 50, } 4463\end{array}$ & $2 c$ \\
\hline 8 & $\begin{array}{c}\text { Chang, M.; Wu, M.; Chen, Y. Org. Lett. } 2013 \text {, 15, } \\
2822\end{array}$ & 2d \\
\hline
\end{tabular}


5. ${ }^{1} \mathrm{H}$ and ${ }^{13} \mathrm{C}$ NMR Spectra of Substrate Acids and Allylating Agents

$1 a$

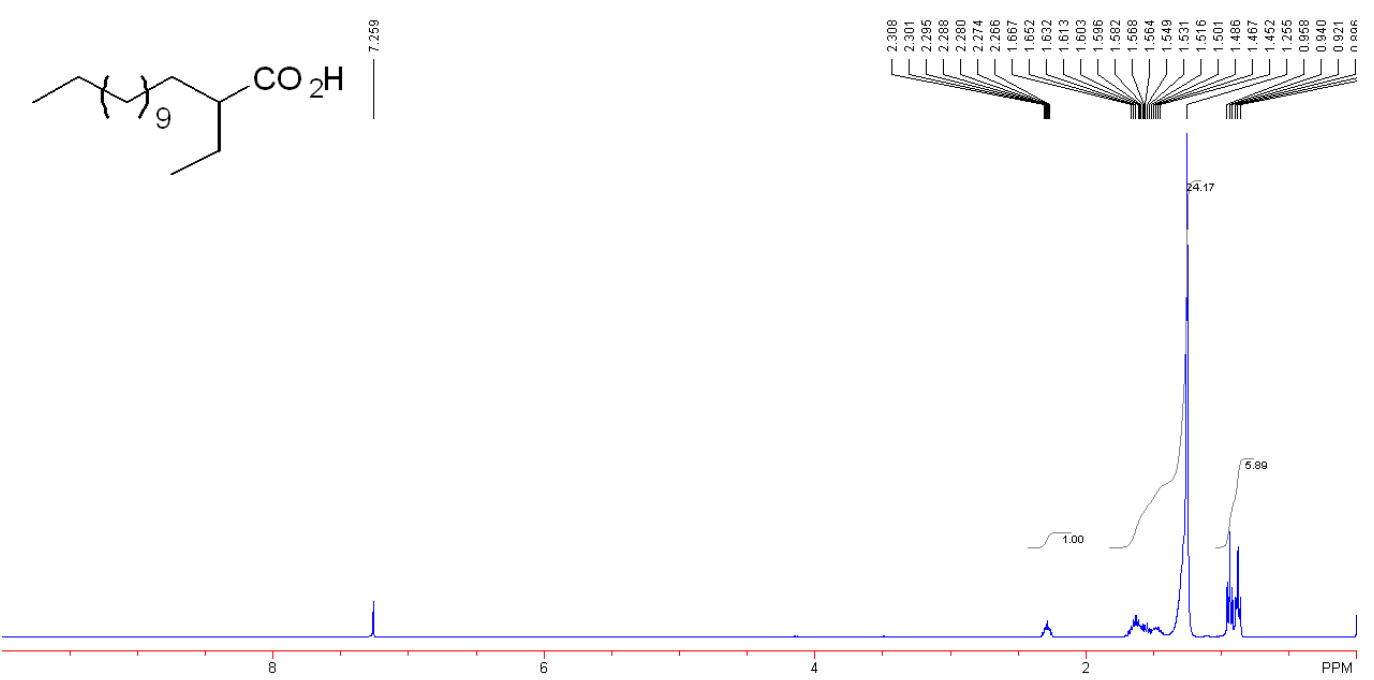

$\mathrm{H}_{9} \mathrm{CO}_{2} \mathrm{H}$
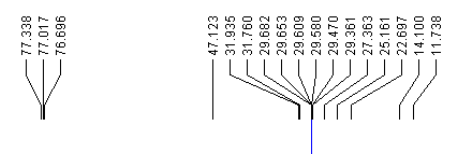

. 
1b<smiles>CCC(Cc1ccccc1)C(=O)O</smiles>

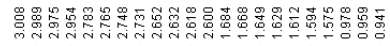

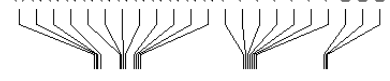
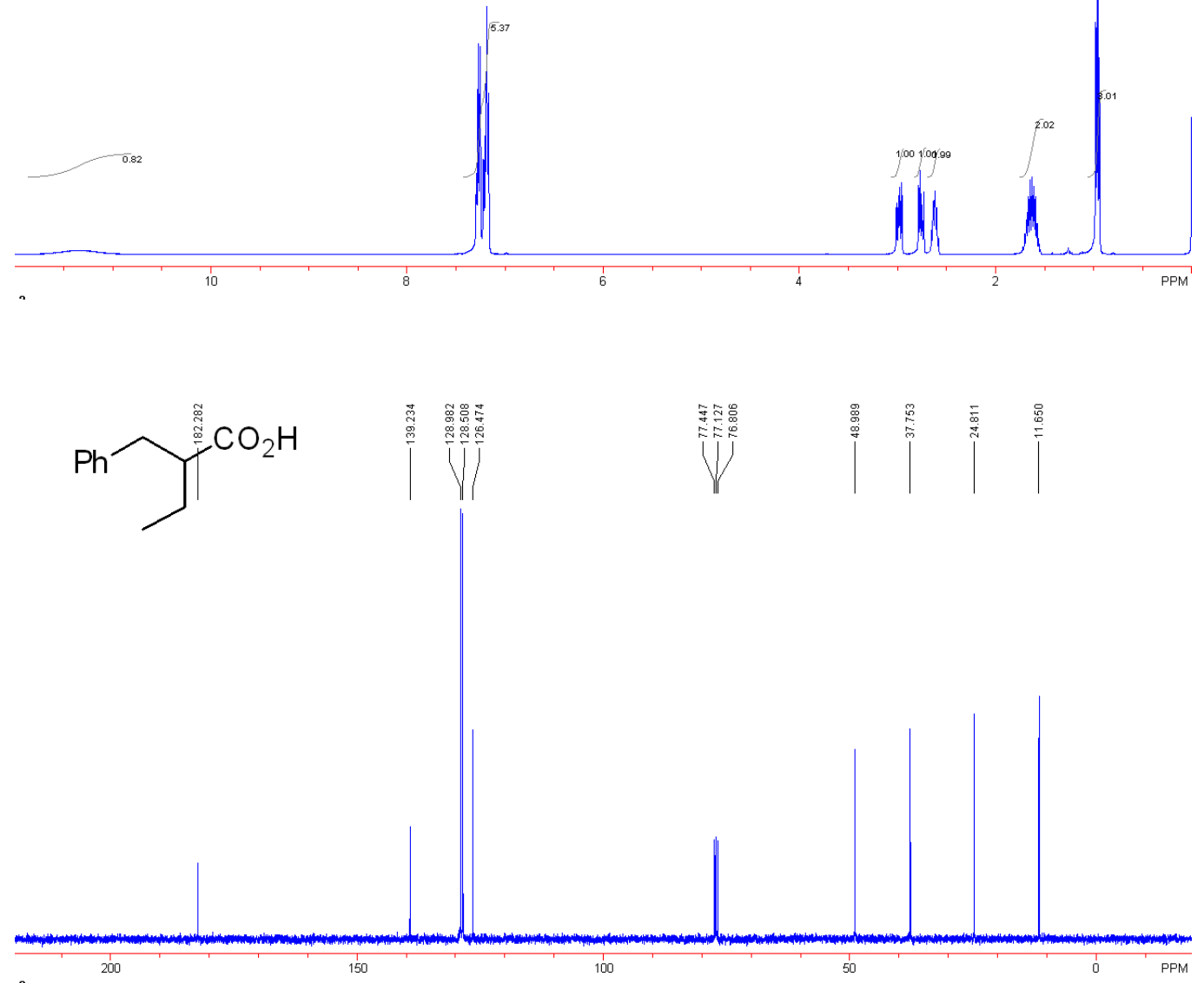
1c<smiles>C1CC1</smiles>
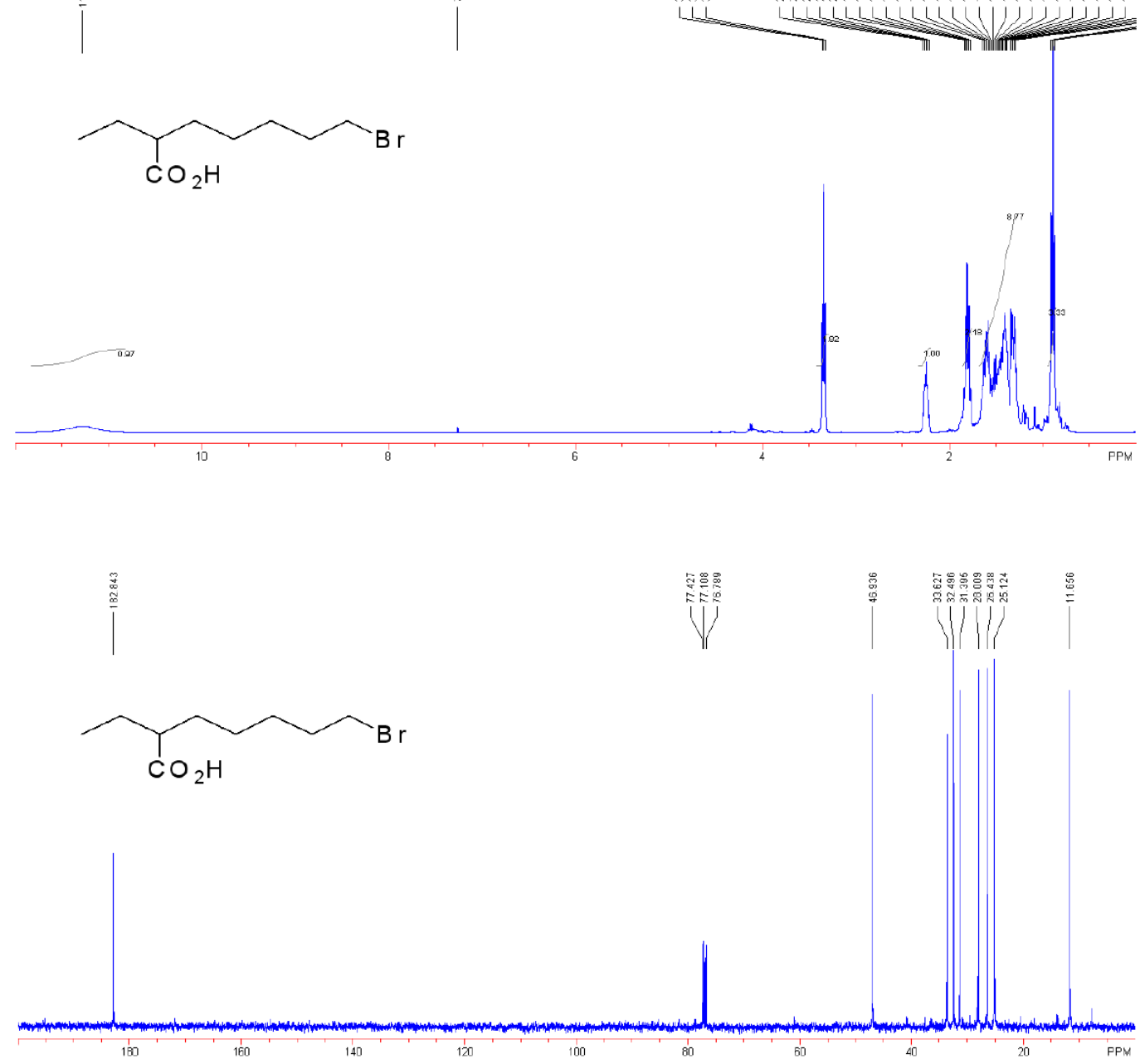


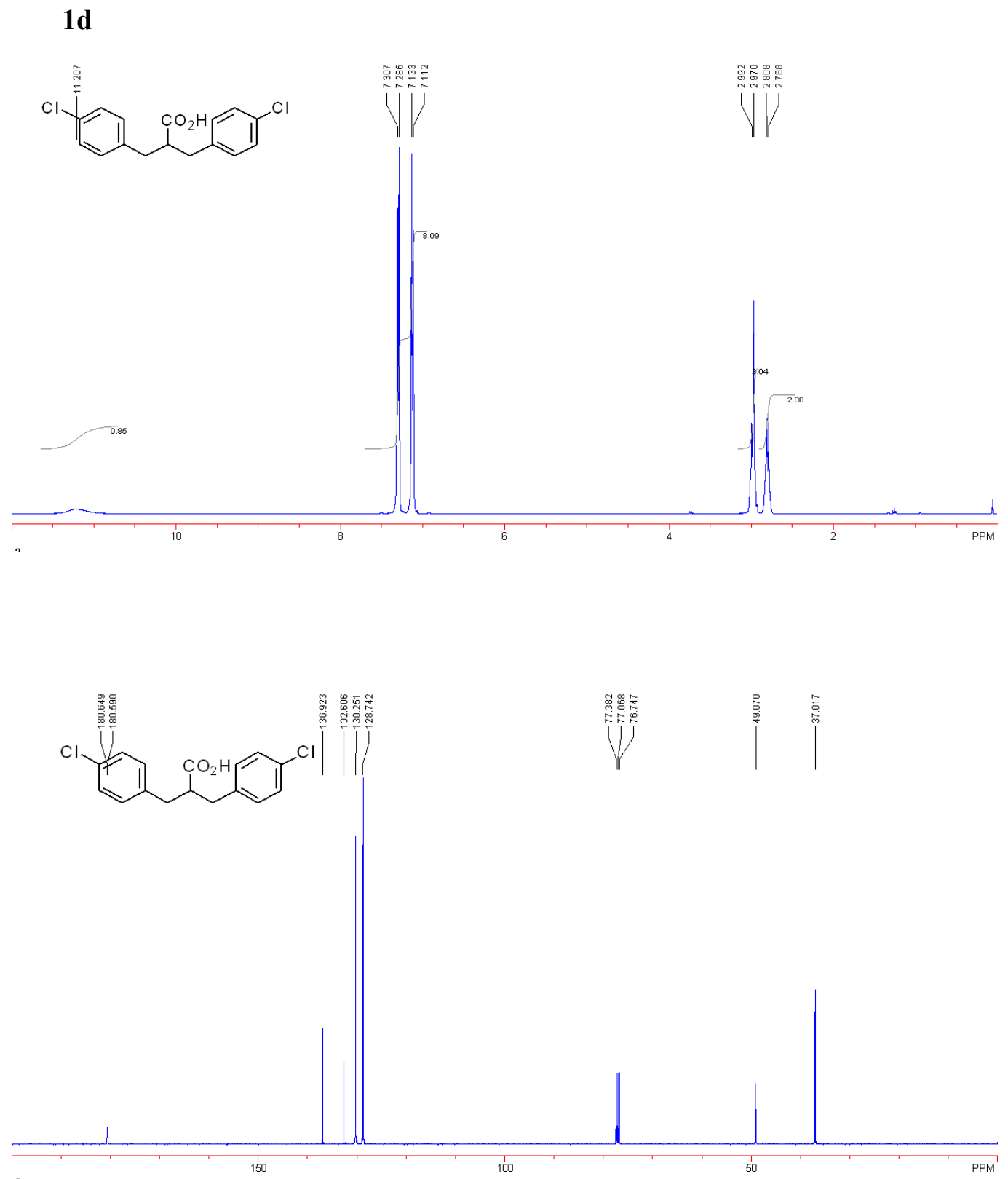




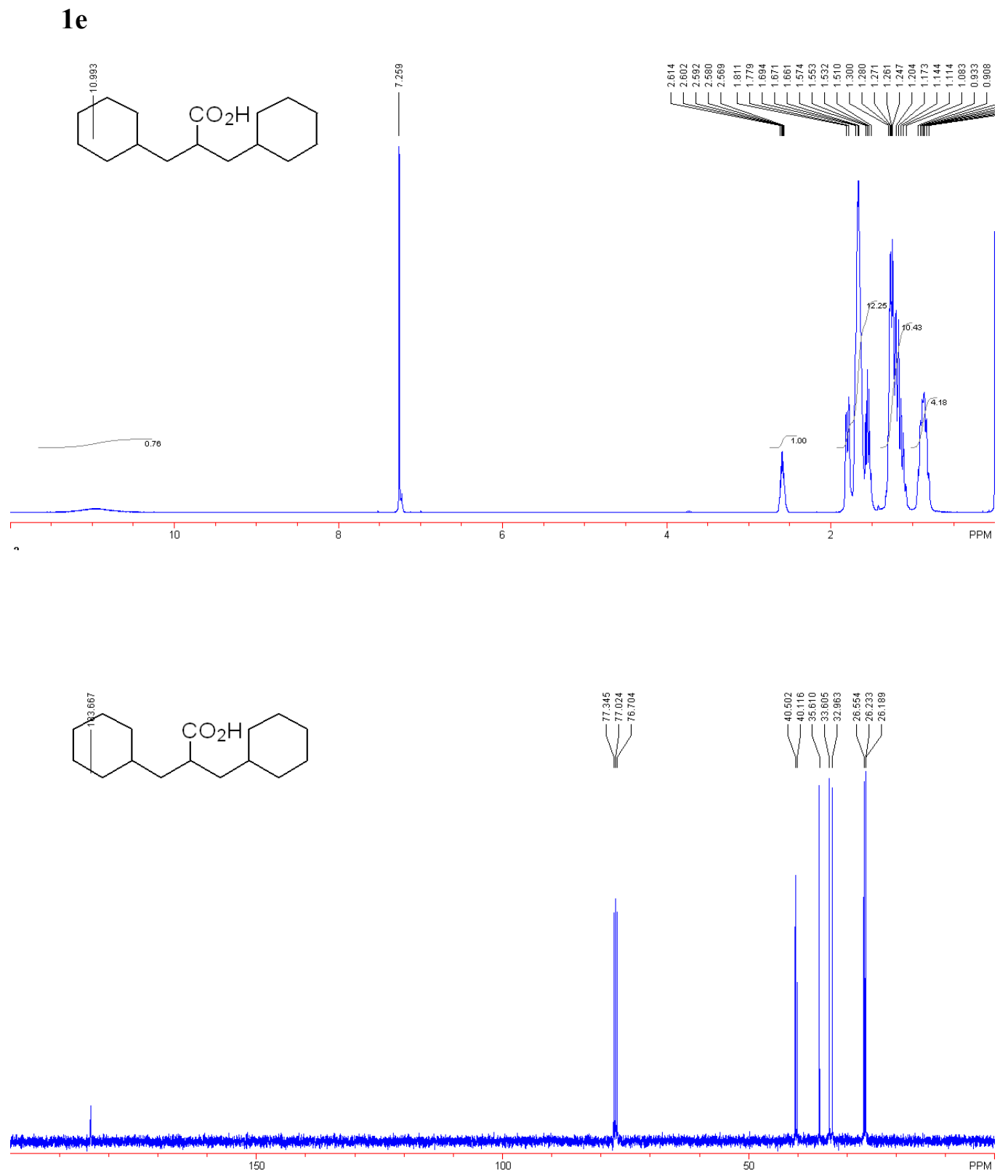


19

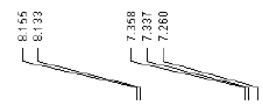

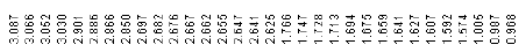

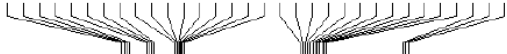

$\overbrace{\mathrm{CO}_{2} \mathrm{H}}$

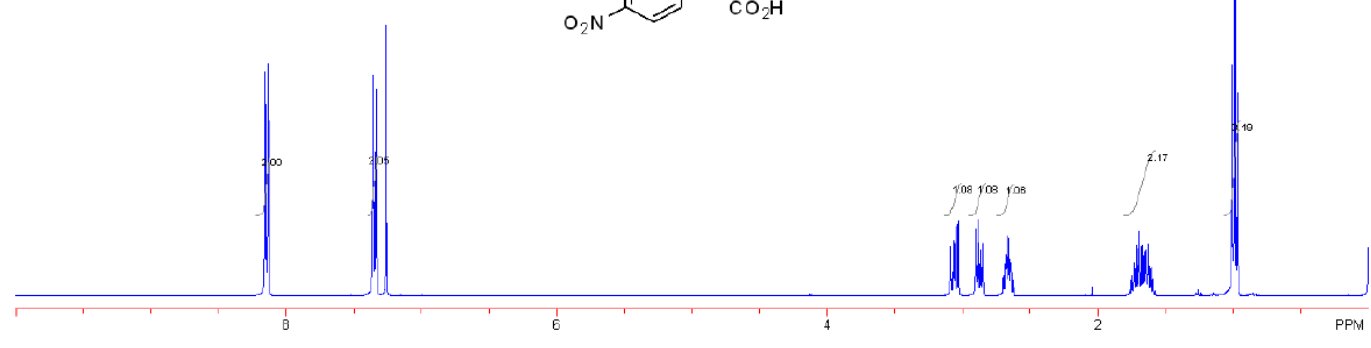

总

$\mathrm{C}_{\mathrm{CO}_{2} \mathrm{H}}$

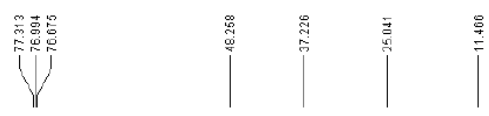



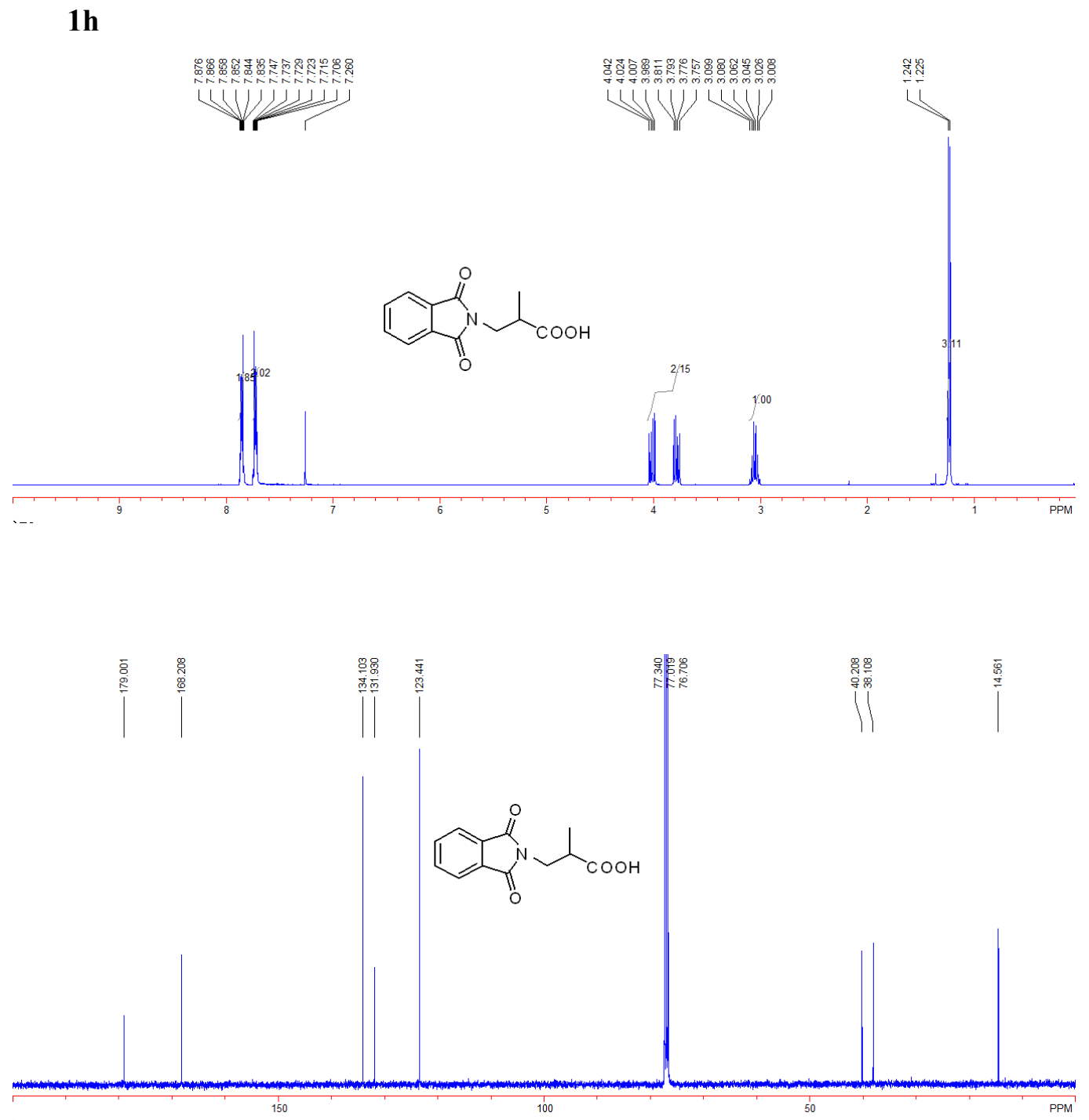
$1 \mathbf{i}$
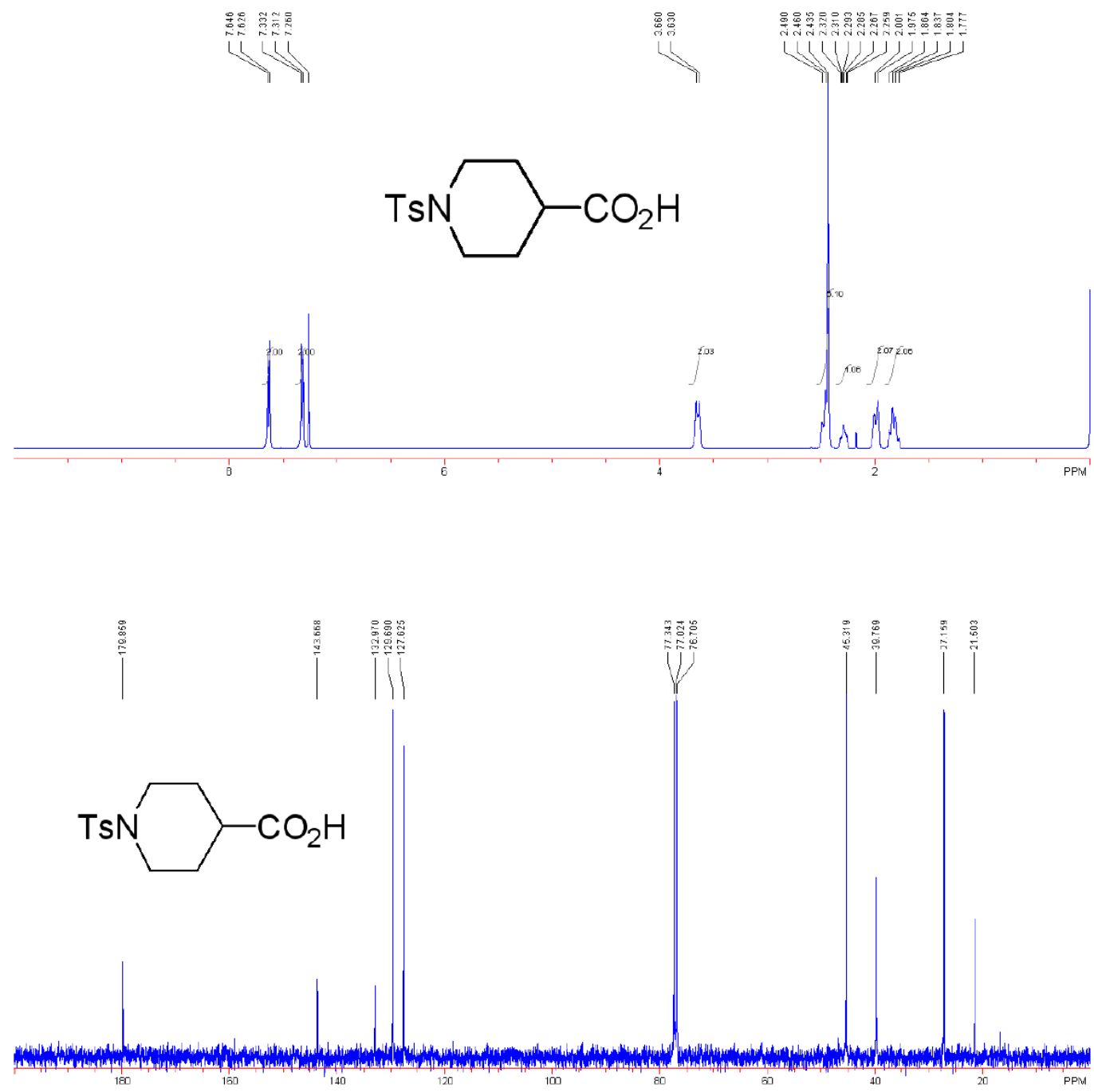


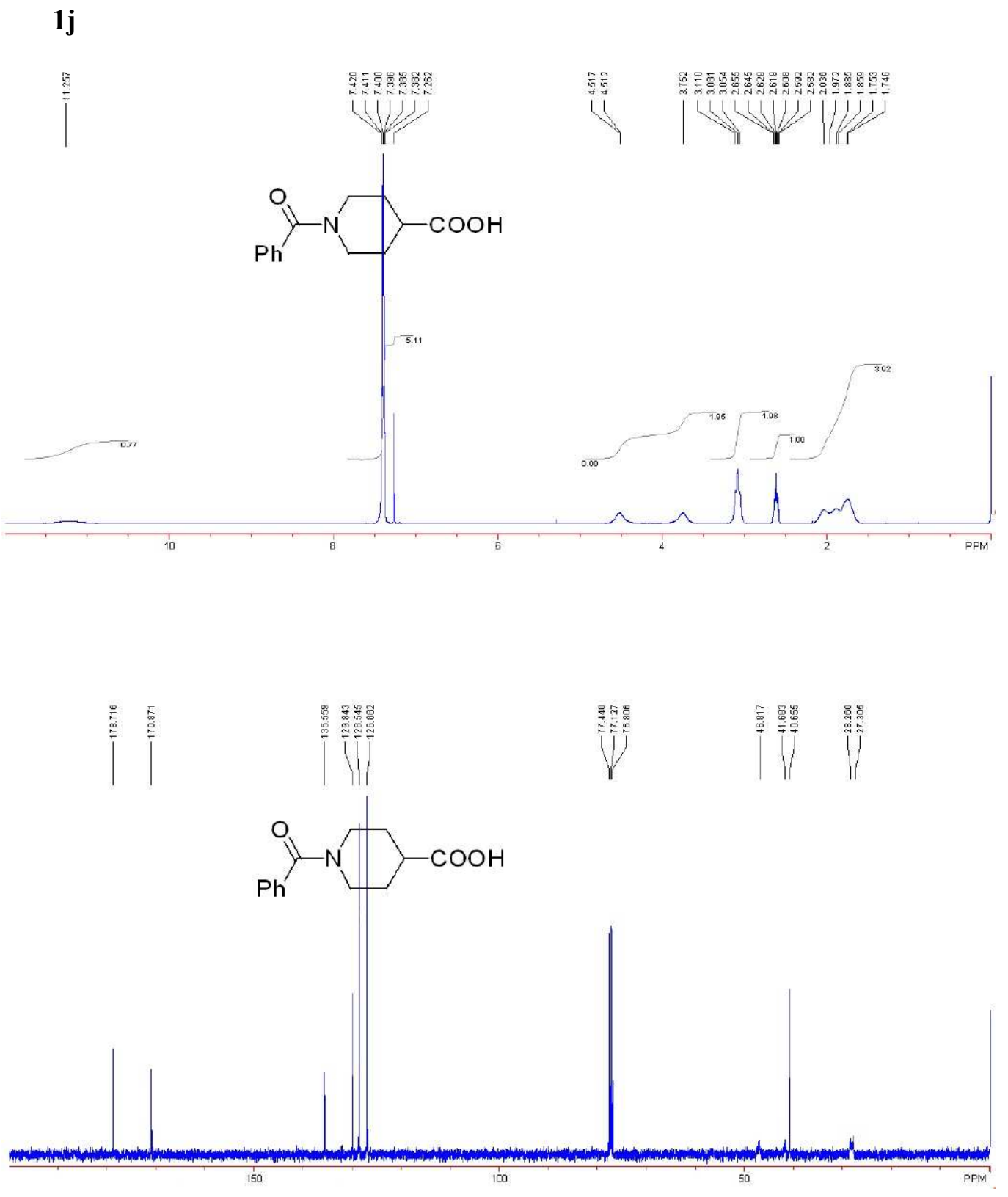


$1 k$

$2 \pi_{0}^{1}$

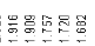

WU

.
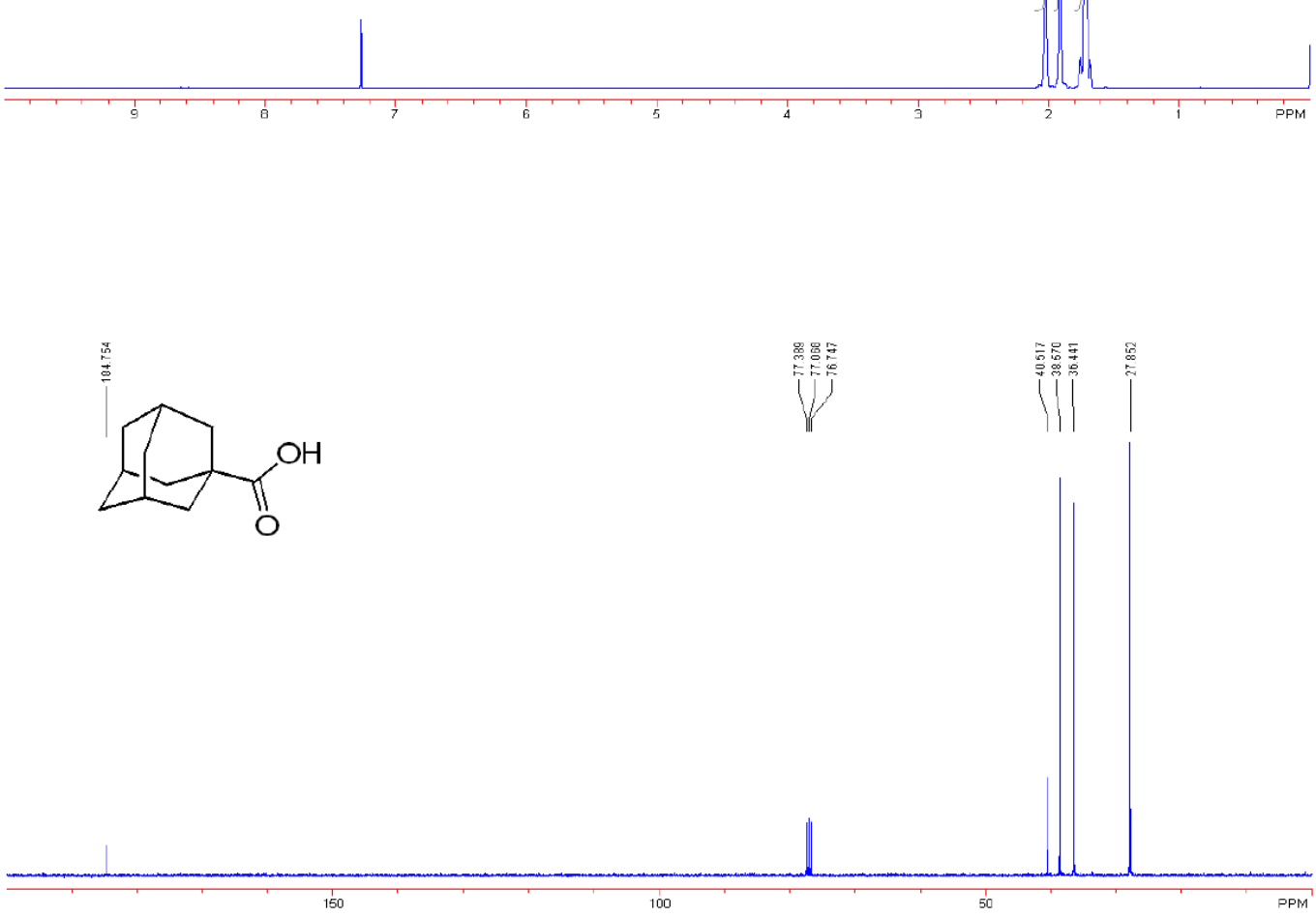
11
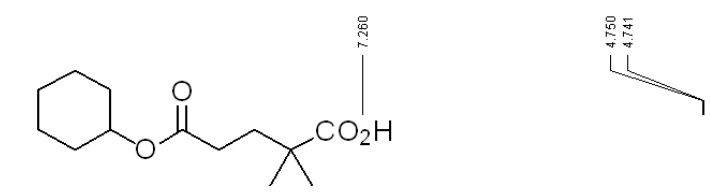

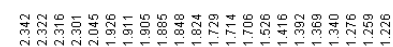

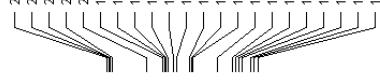
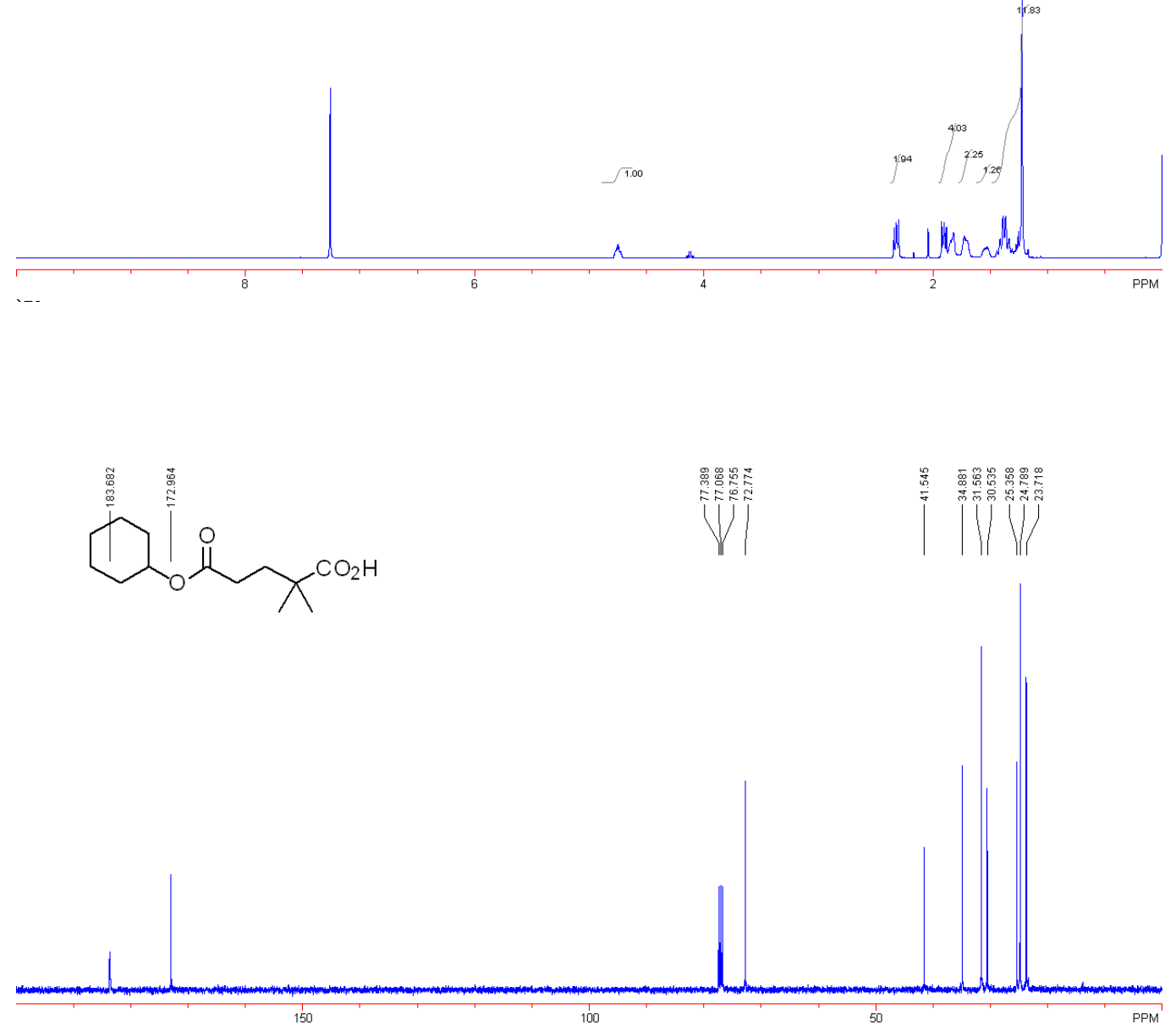

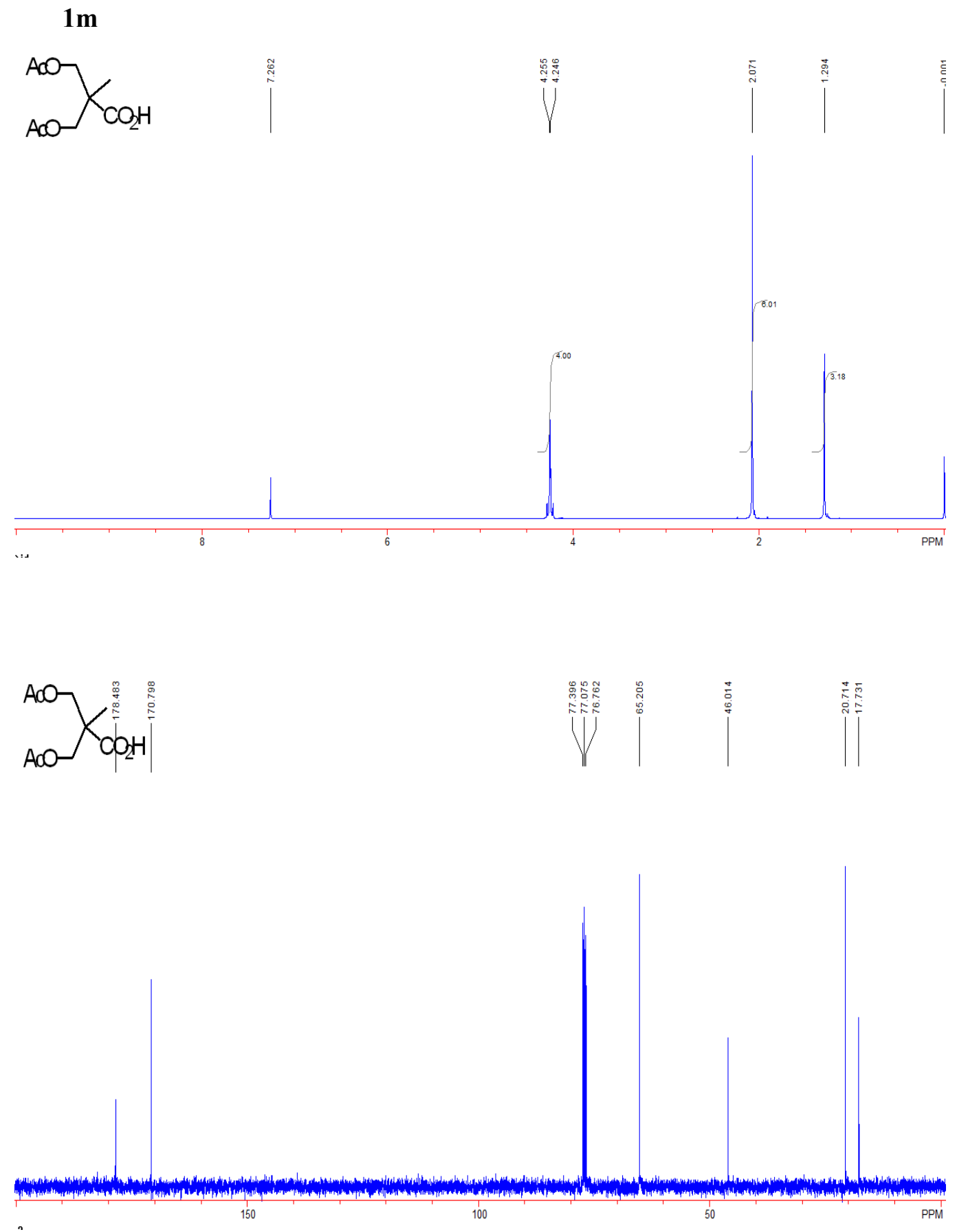
1n
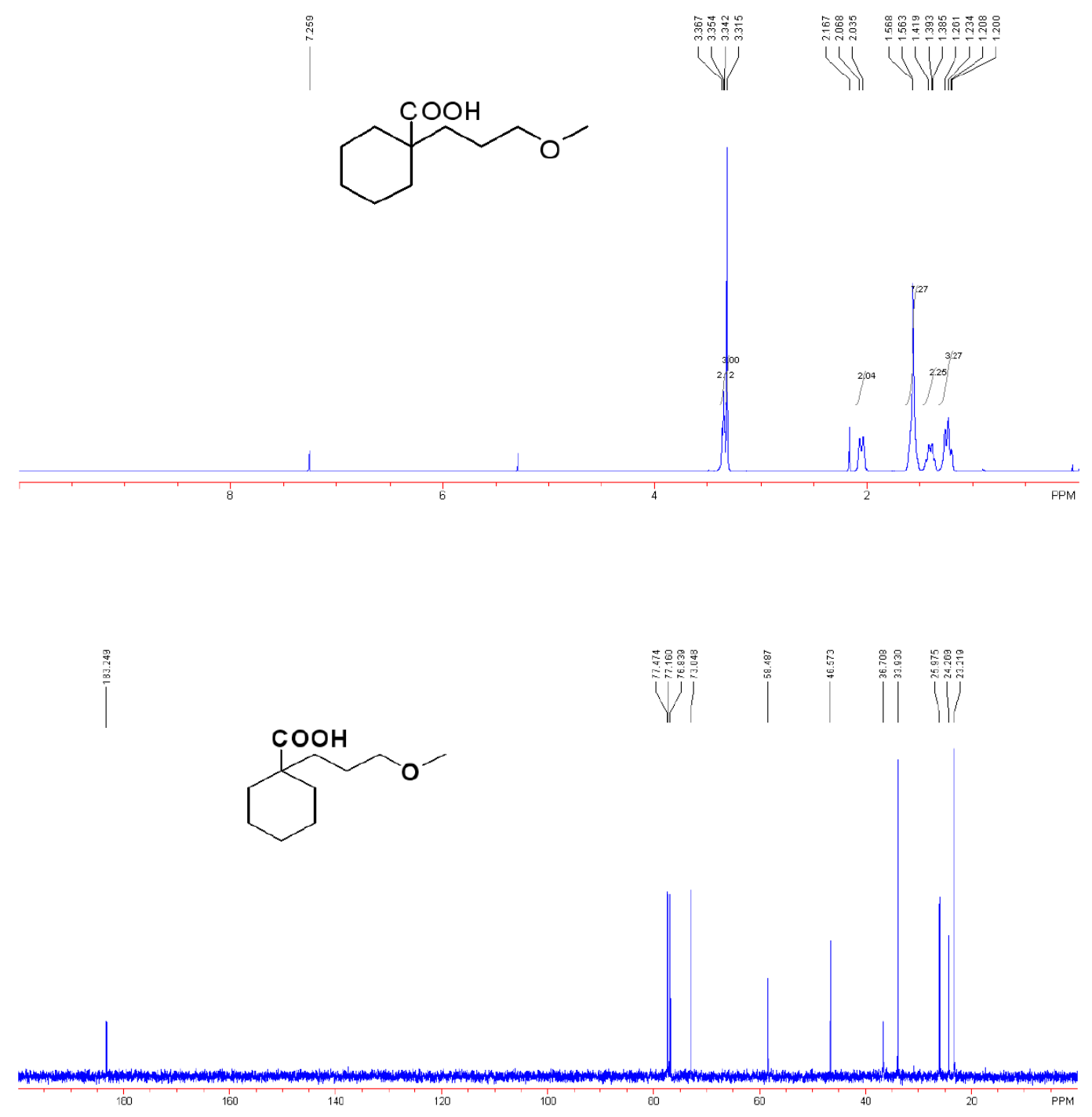
10

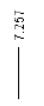

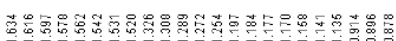
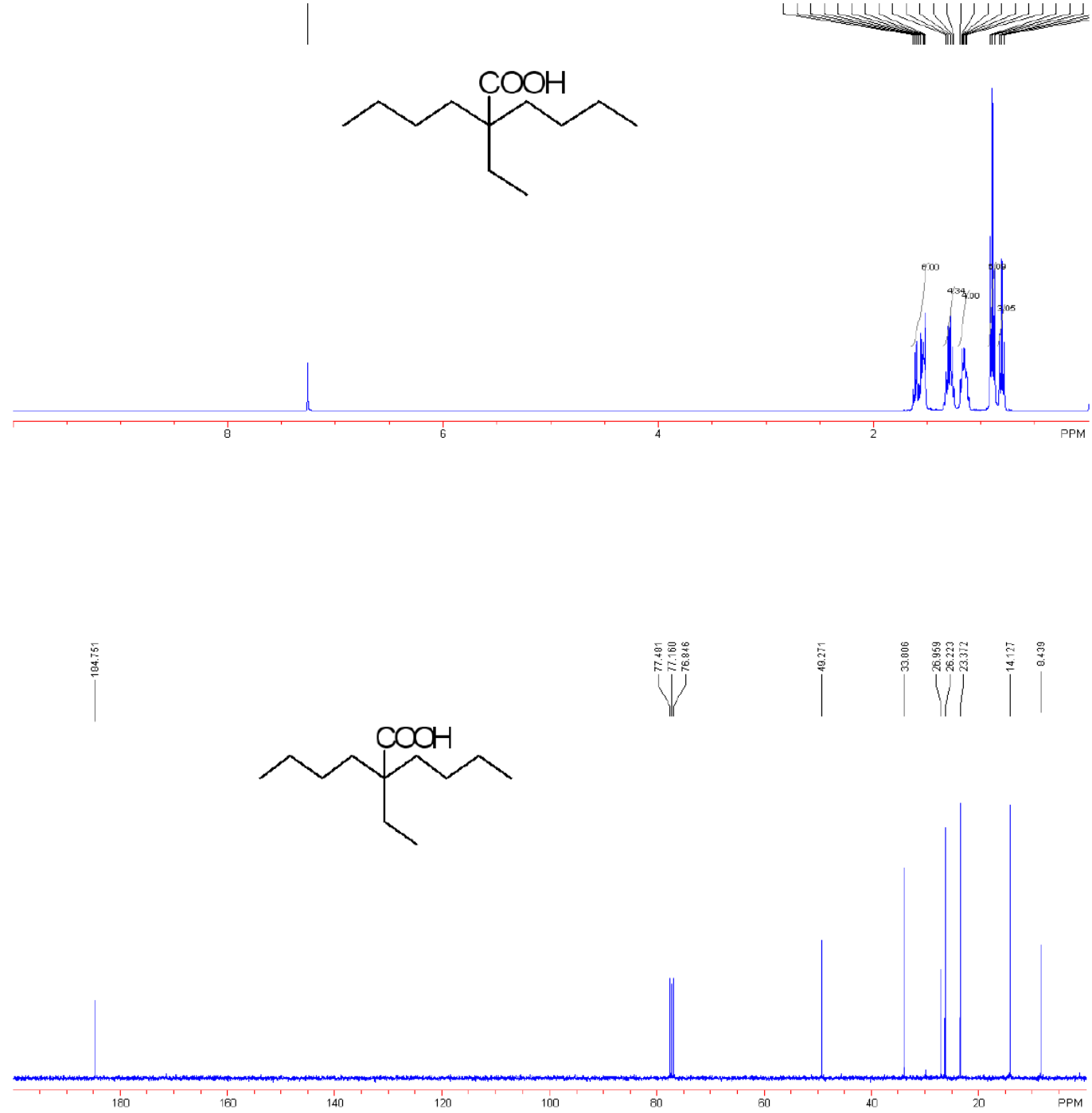

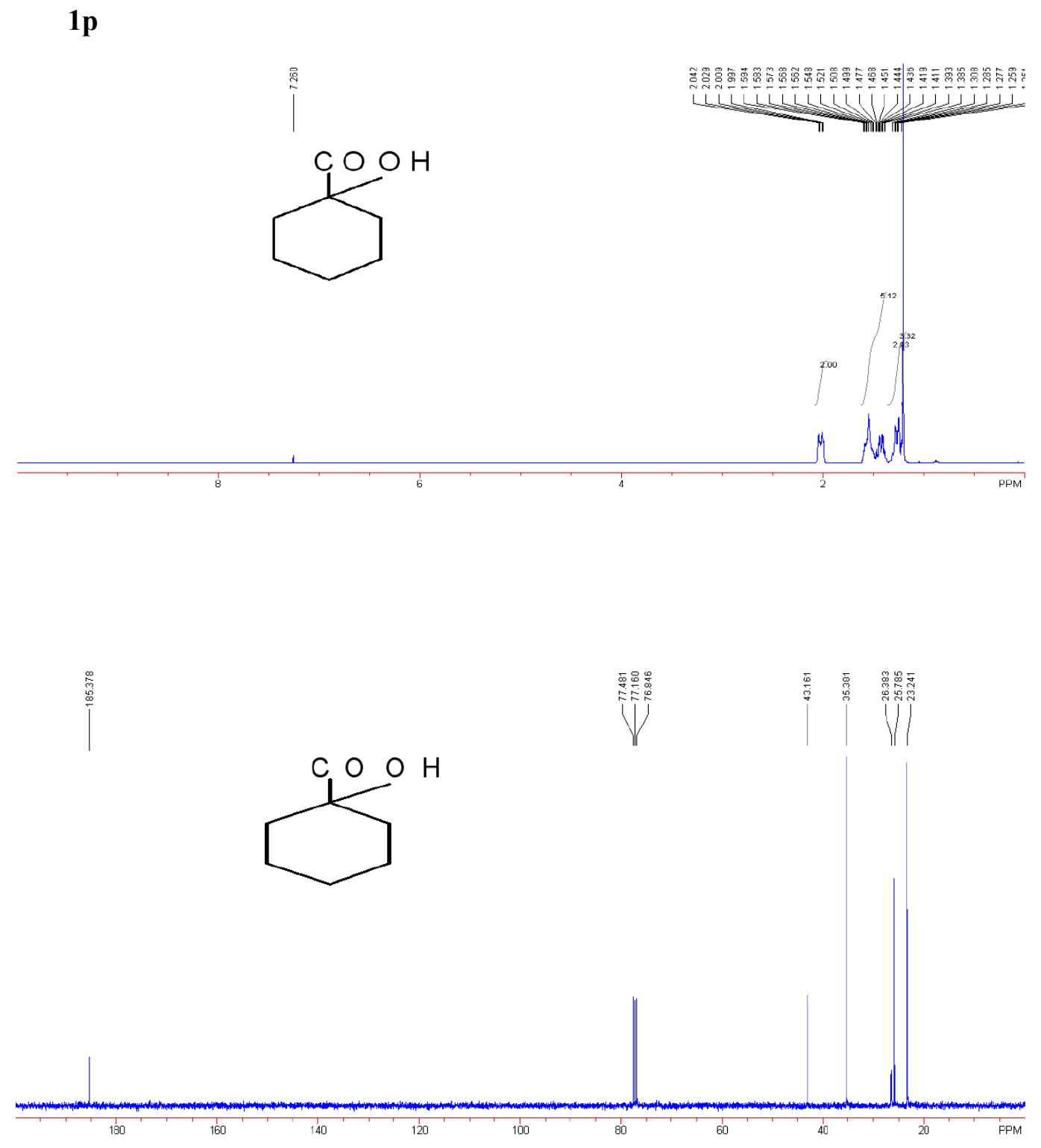


\section{$1 q$}

$\mathrm{C}^{\mathrm{CO}} \mathrm{C}_{7} \mathrm{H}$

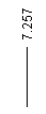

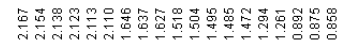

uW

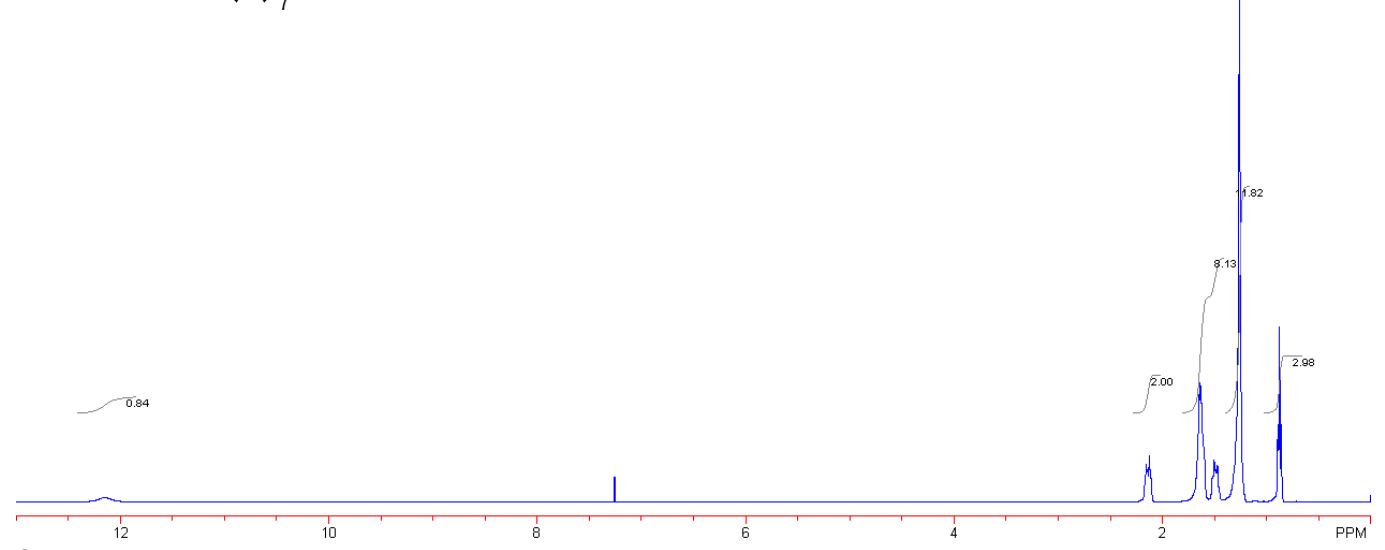

$\overbrace{\left(y_{7}\right.}^{\mathrm{CO}_{2} \mathrm{H}}$

V|

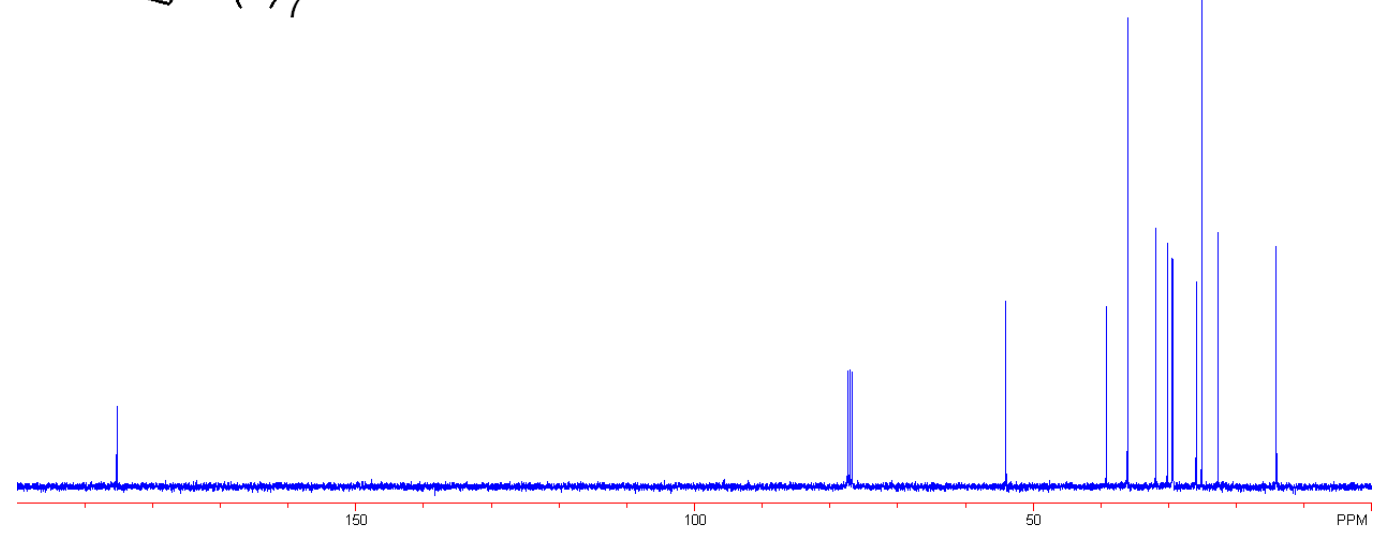



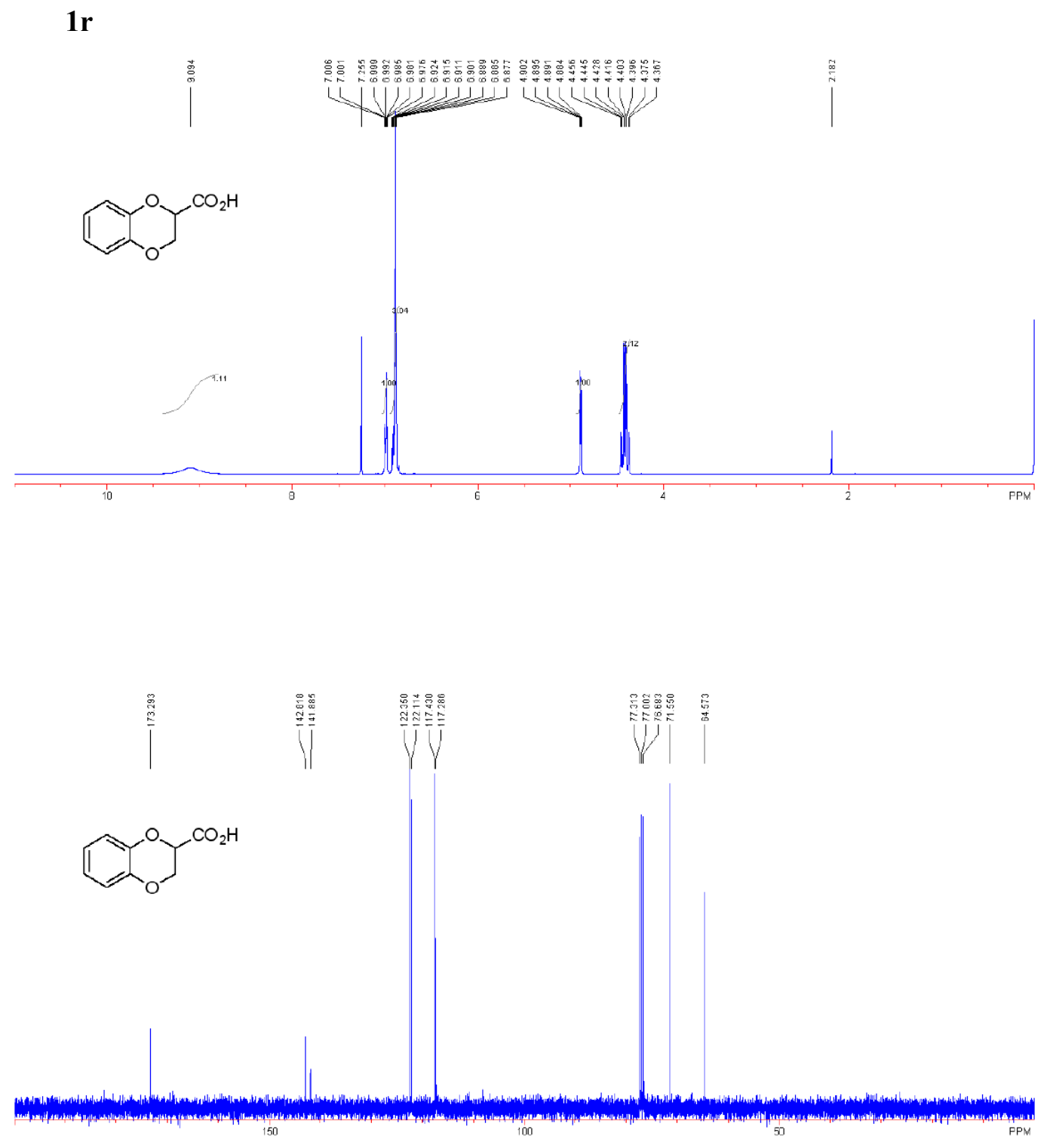
$1 s$
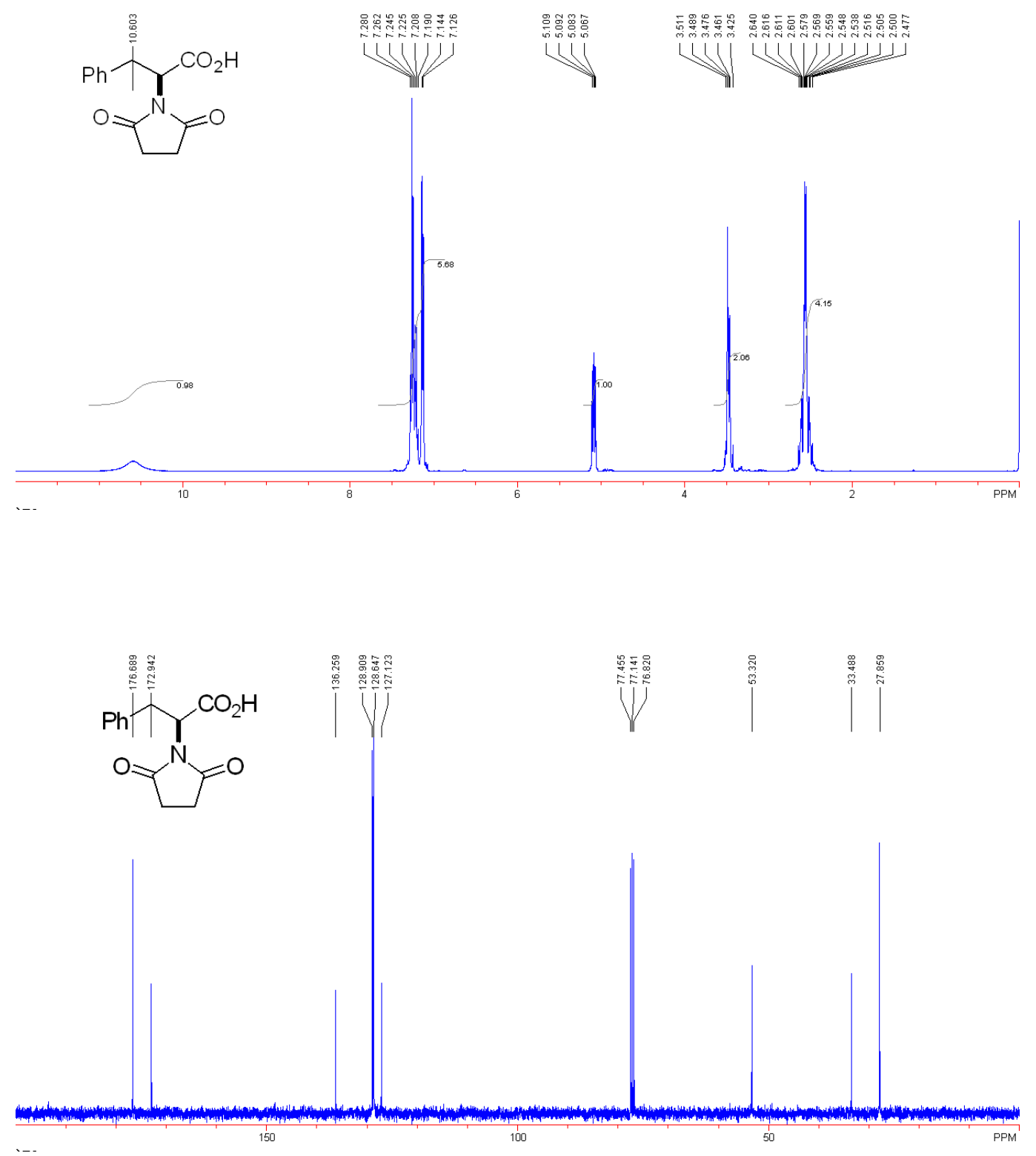
$1 t$<smiles>O=C(O)CN1C(=O)c2ccccc2C1=O</smiles>

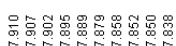

WWH

声

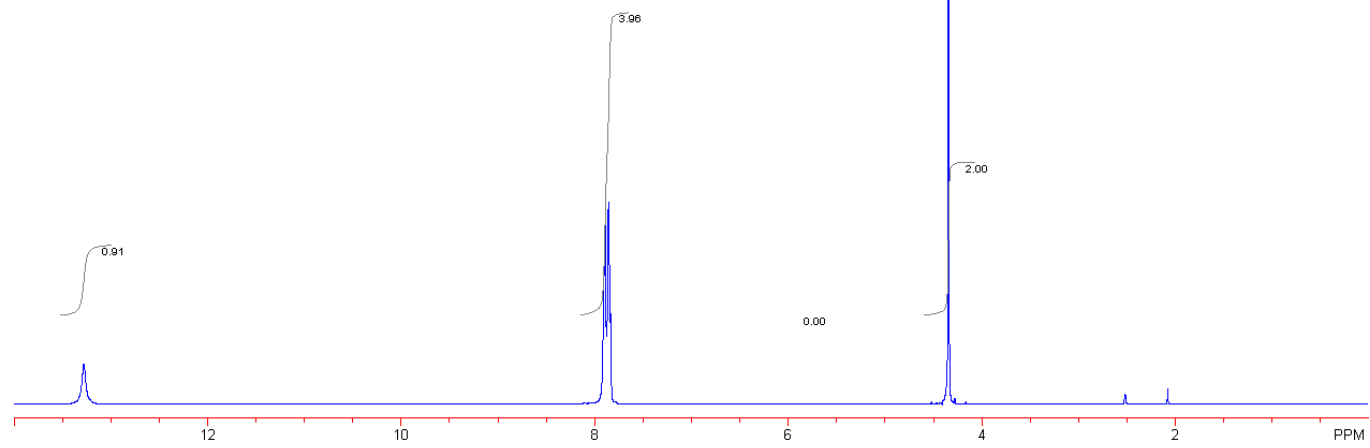

$\underbrace{\mathrm{O}}_{\mathrm{N}-\mathrm{CO}_{2} \mathrm{H}}$

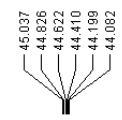

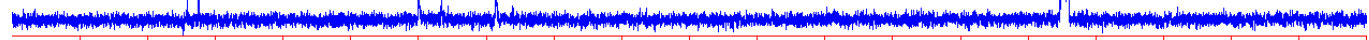


$1 u$
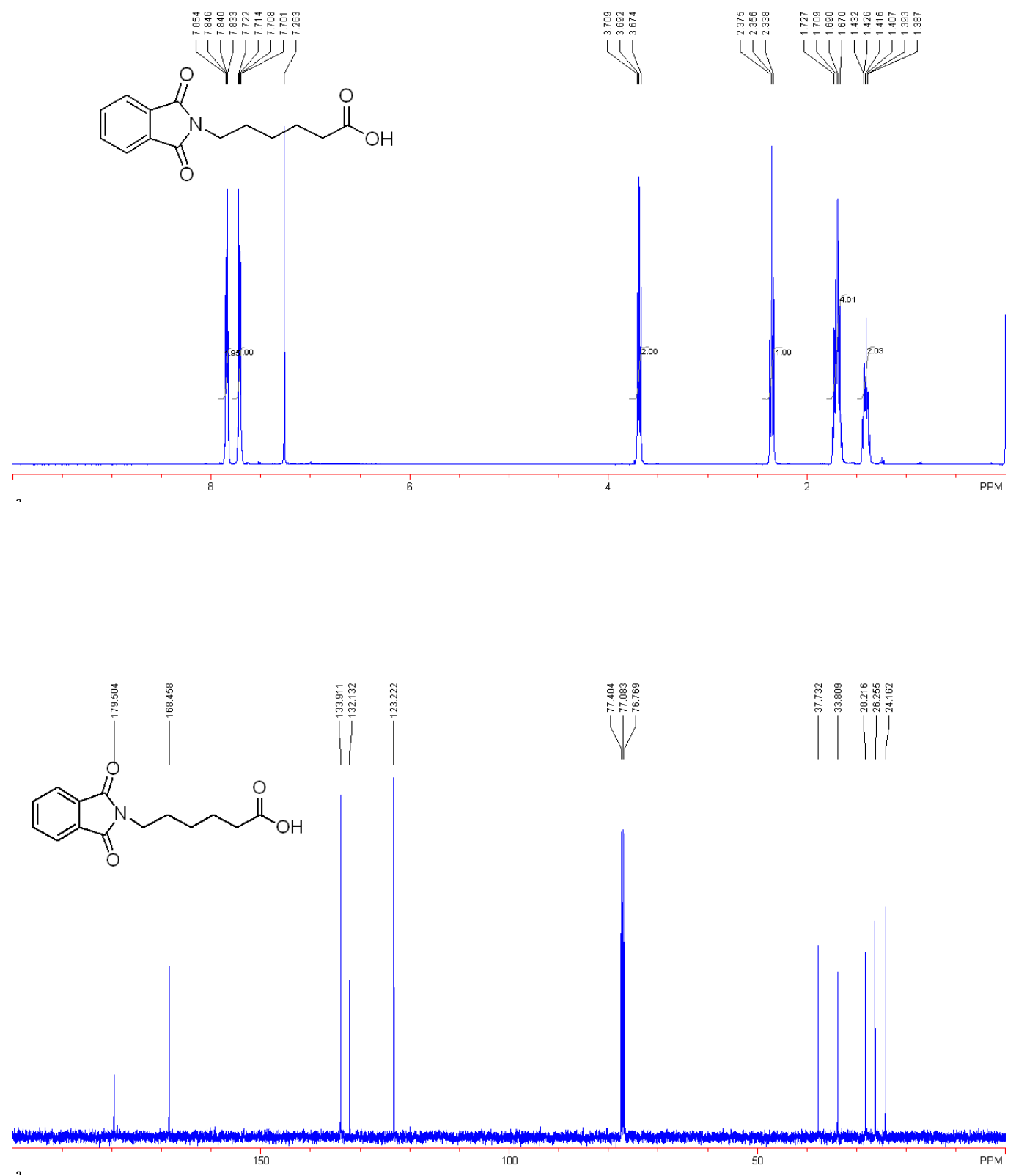


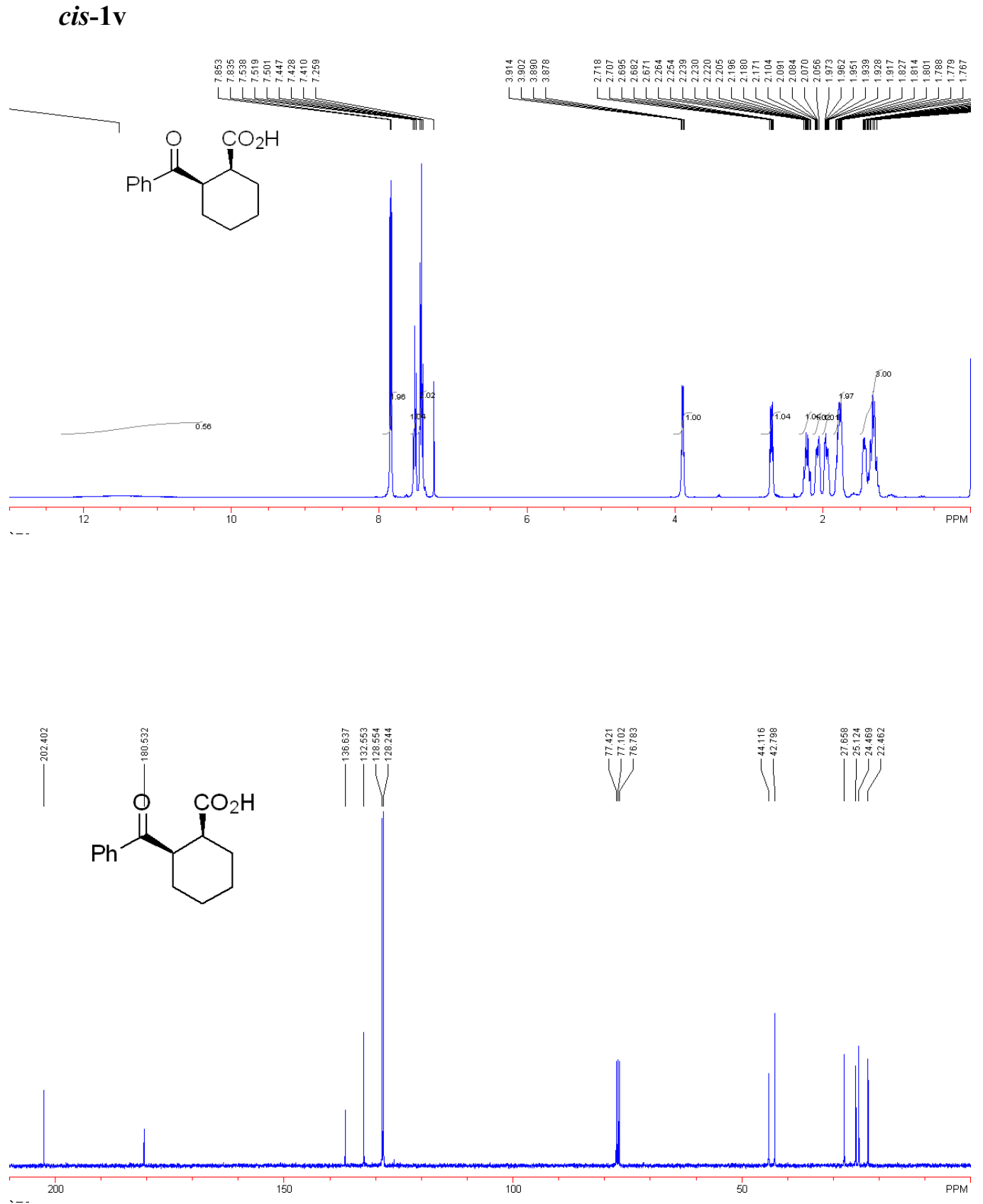




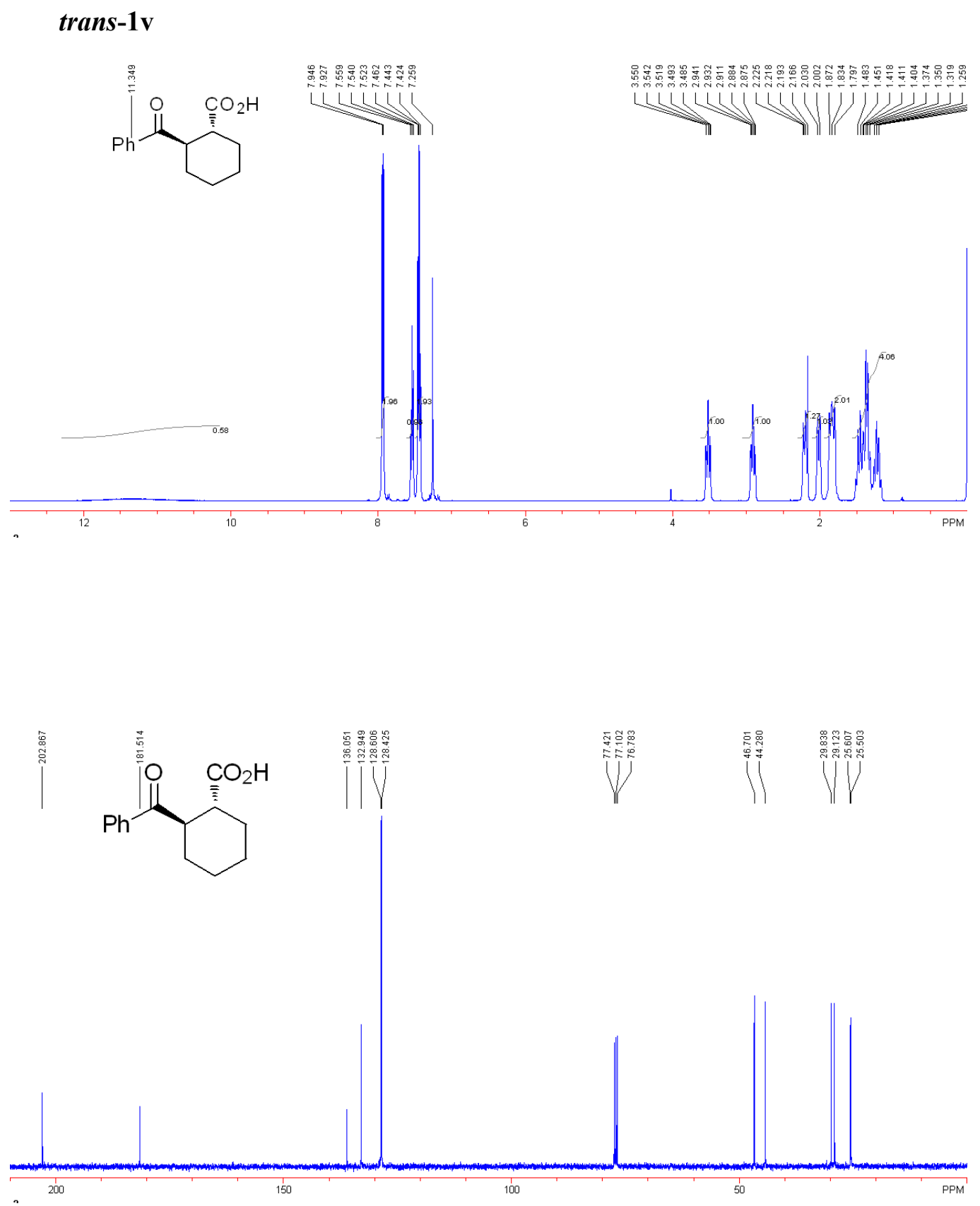


1w
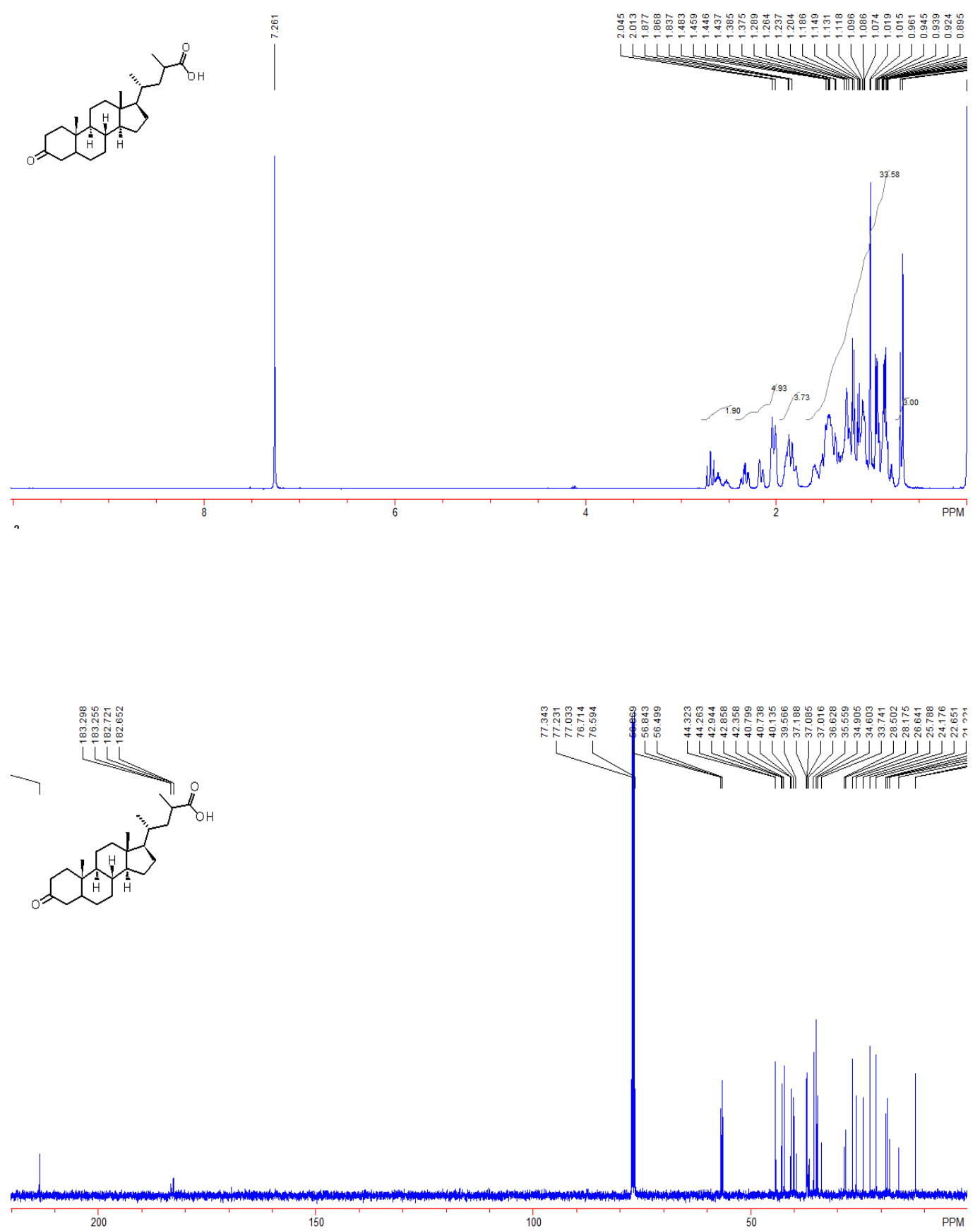
$2 a$
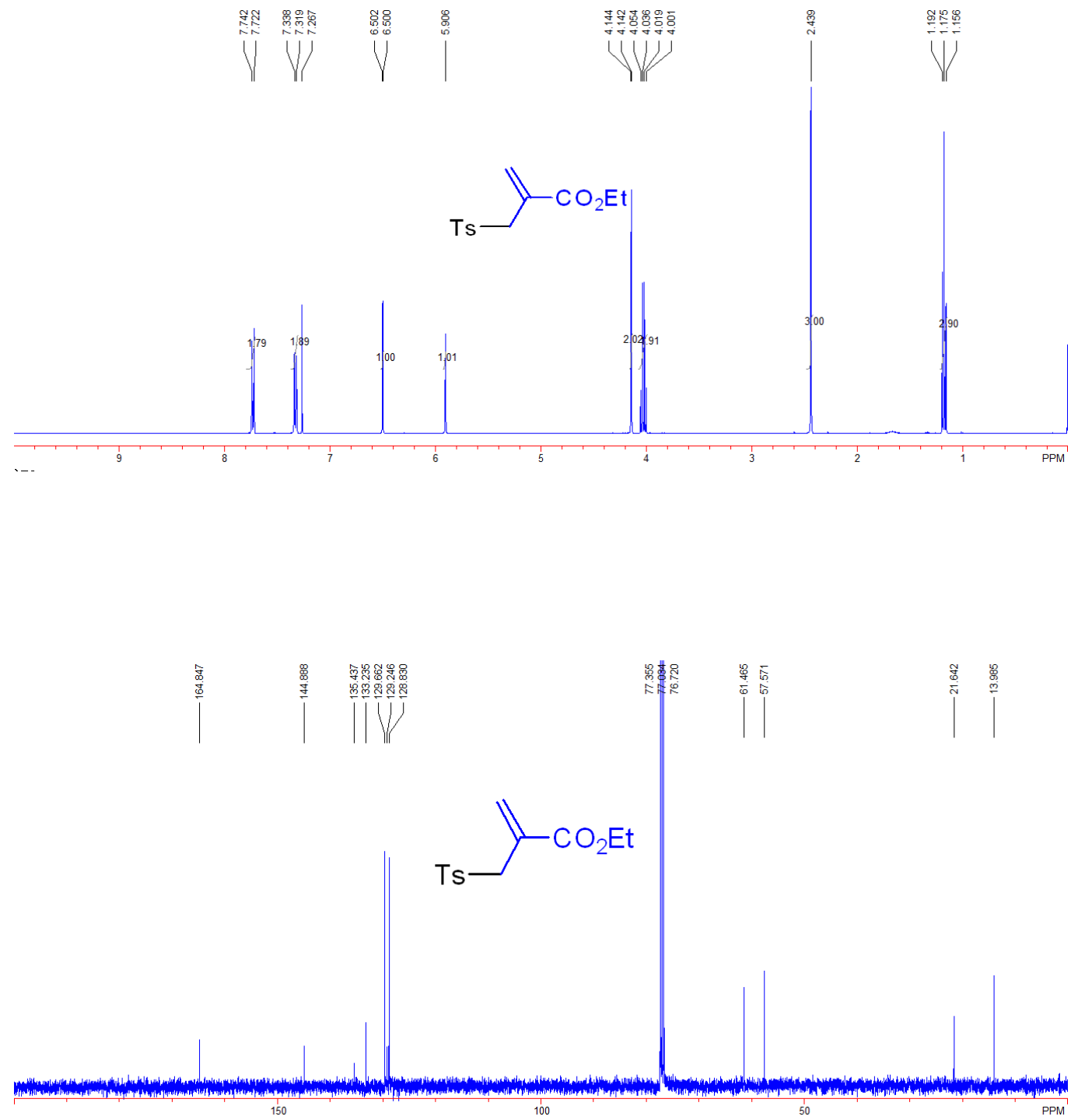
2b
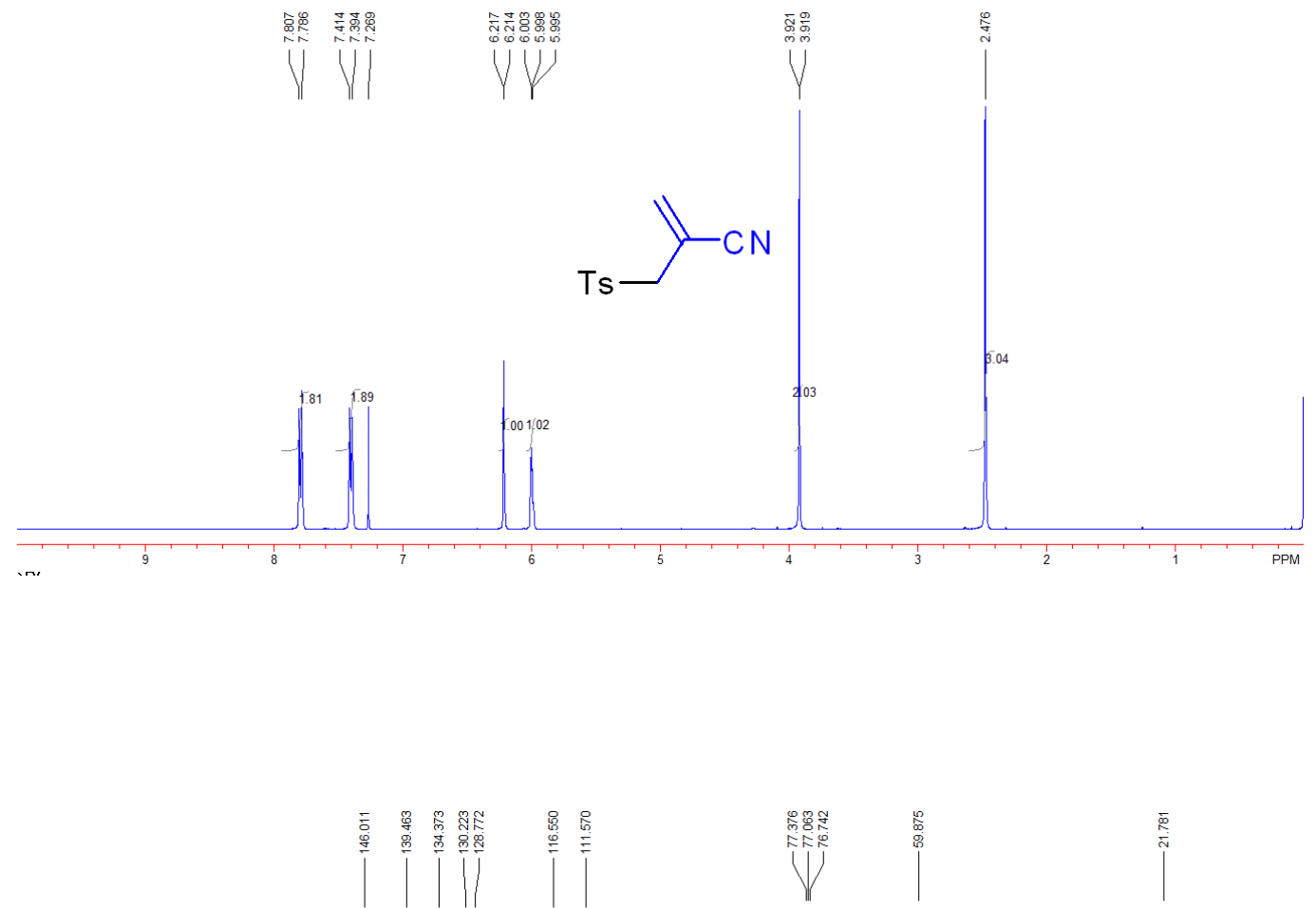

$y_{\mathrm{T}} \mathrm{CN}$

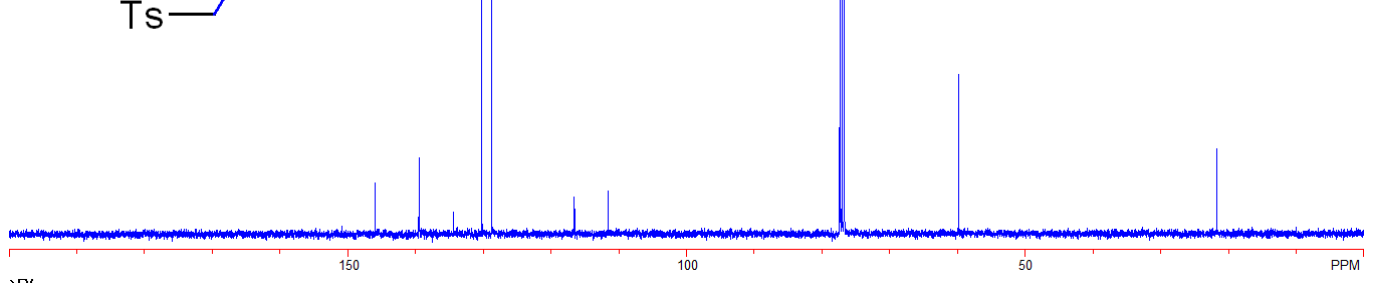


2c

诲

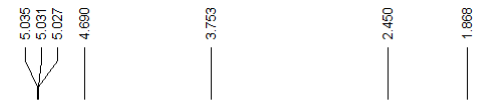
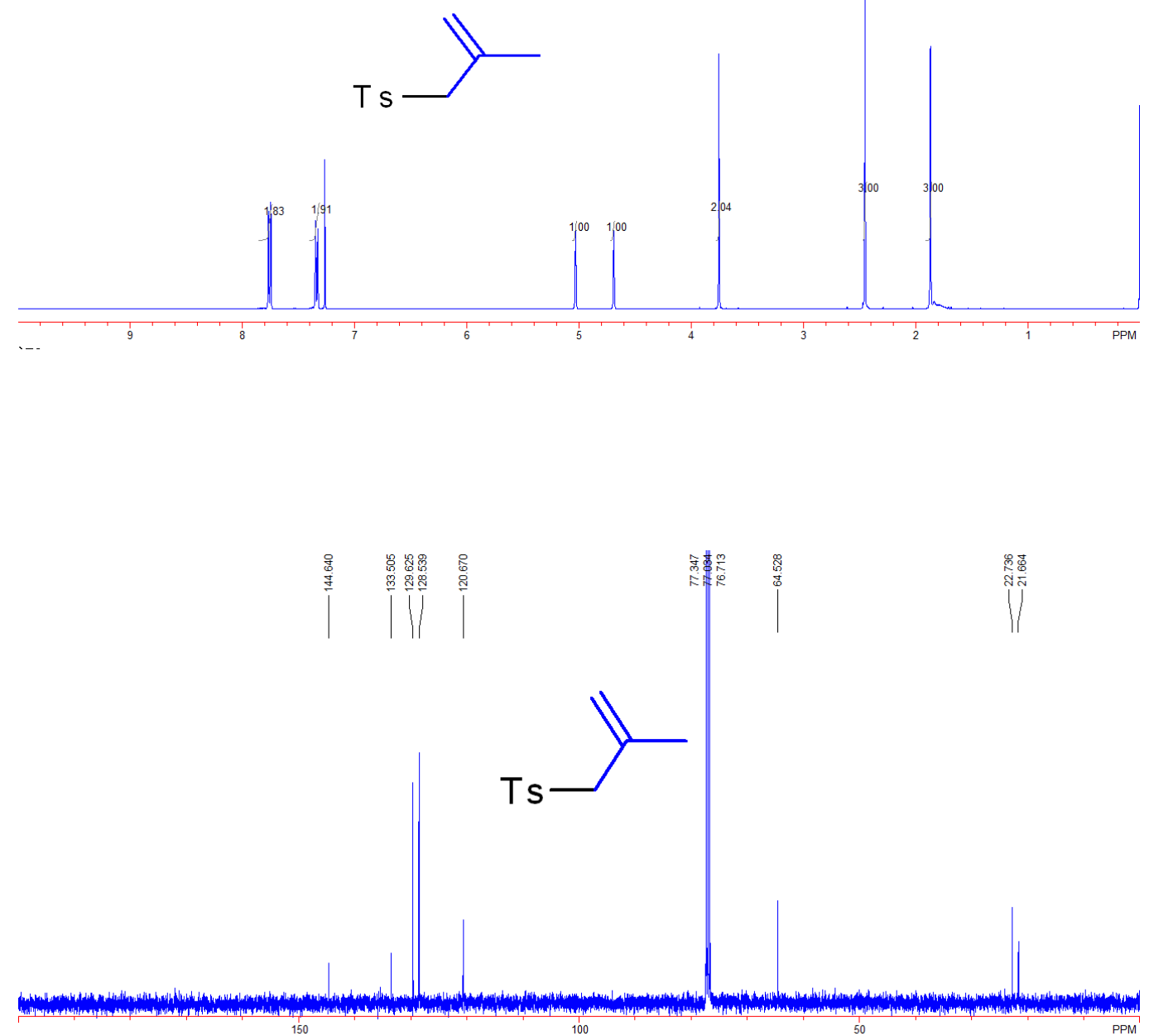
2d

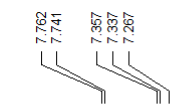

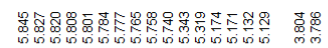

Will

$\sum_{S}$
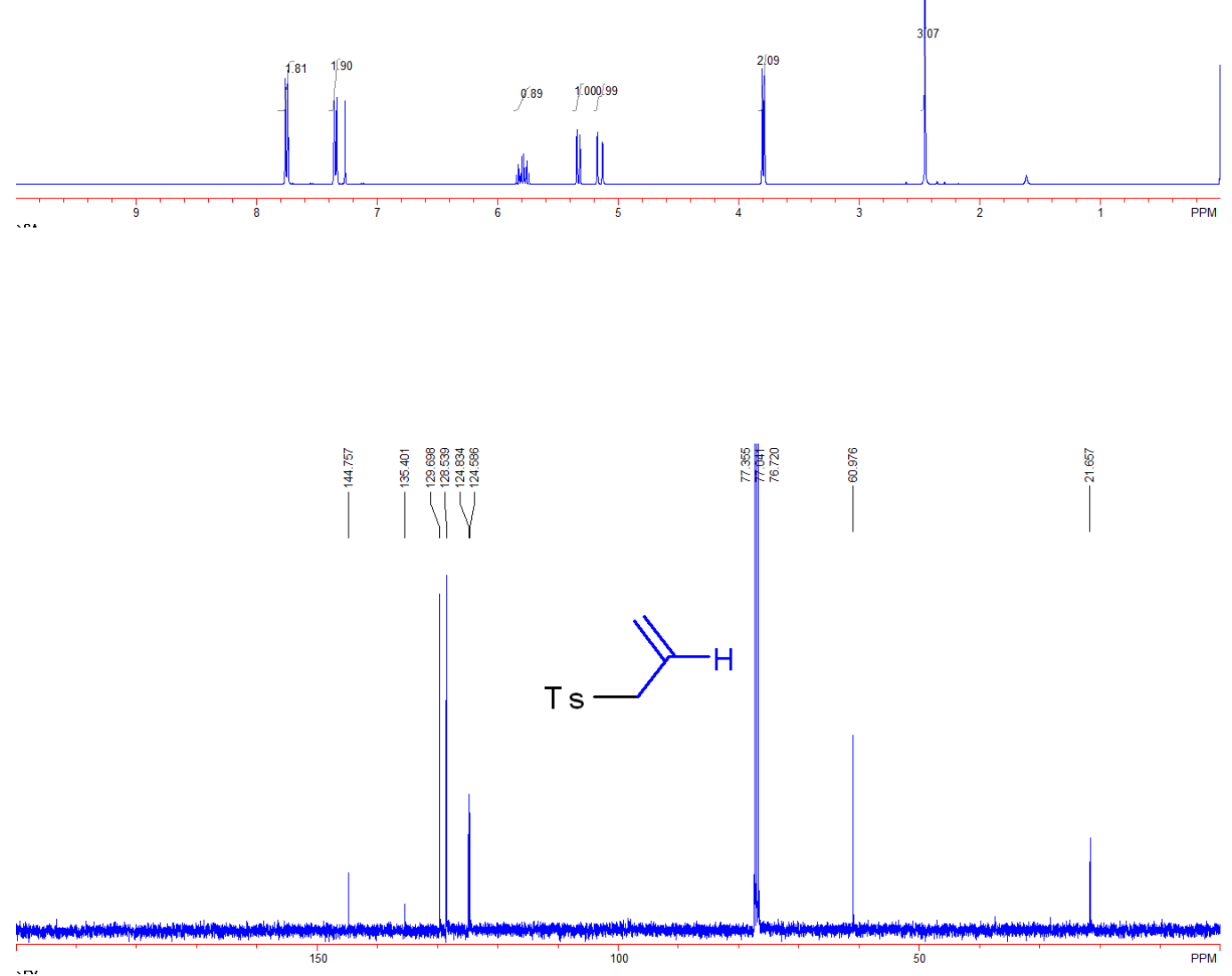
$2 e$
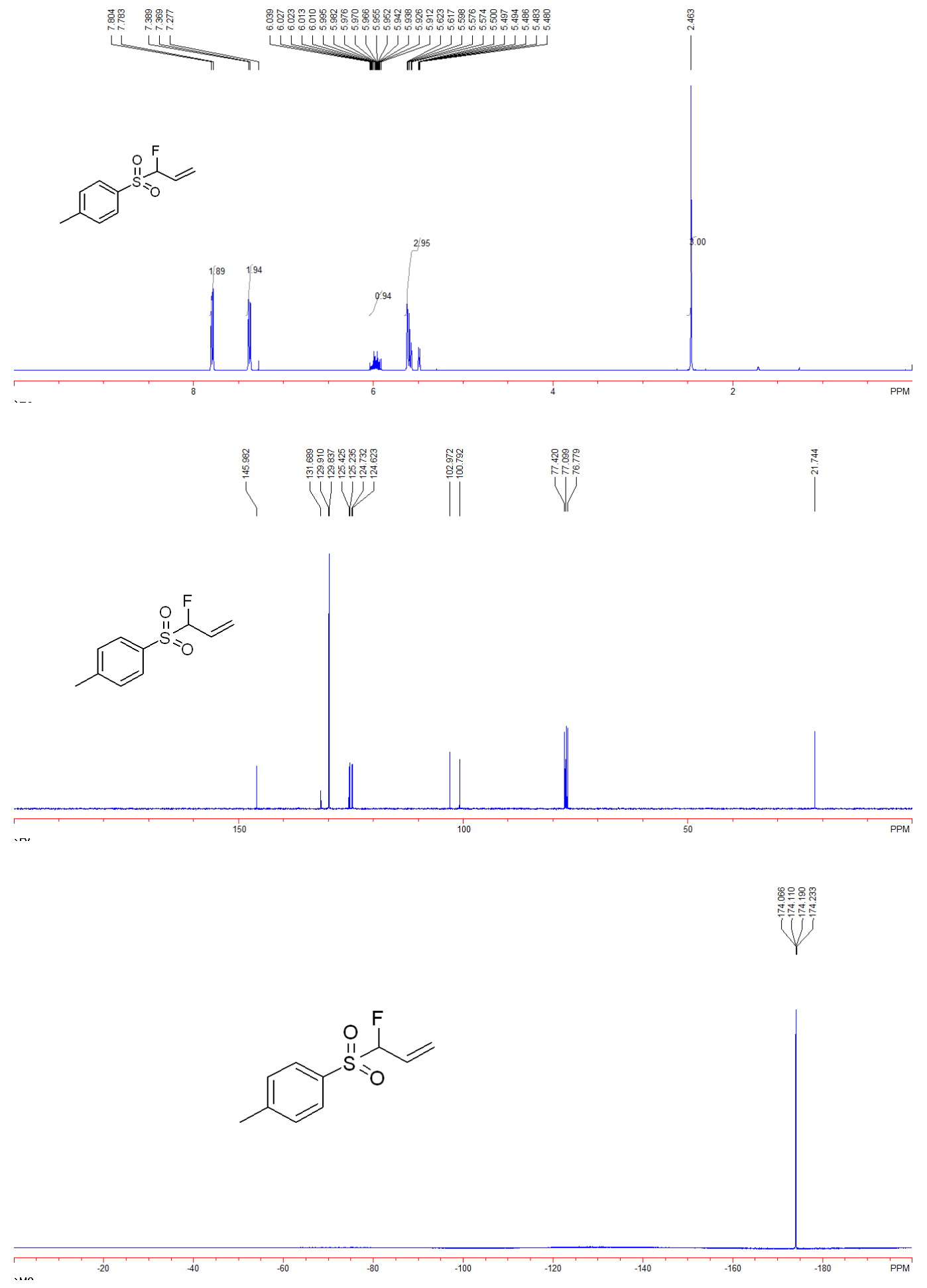


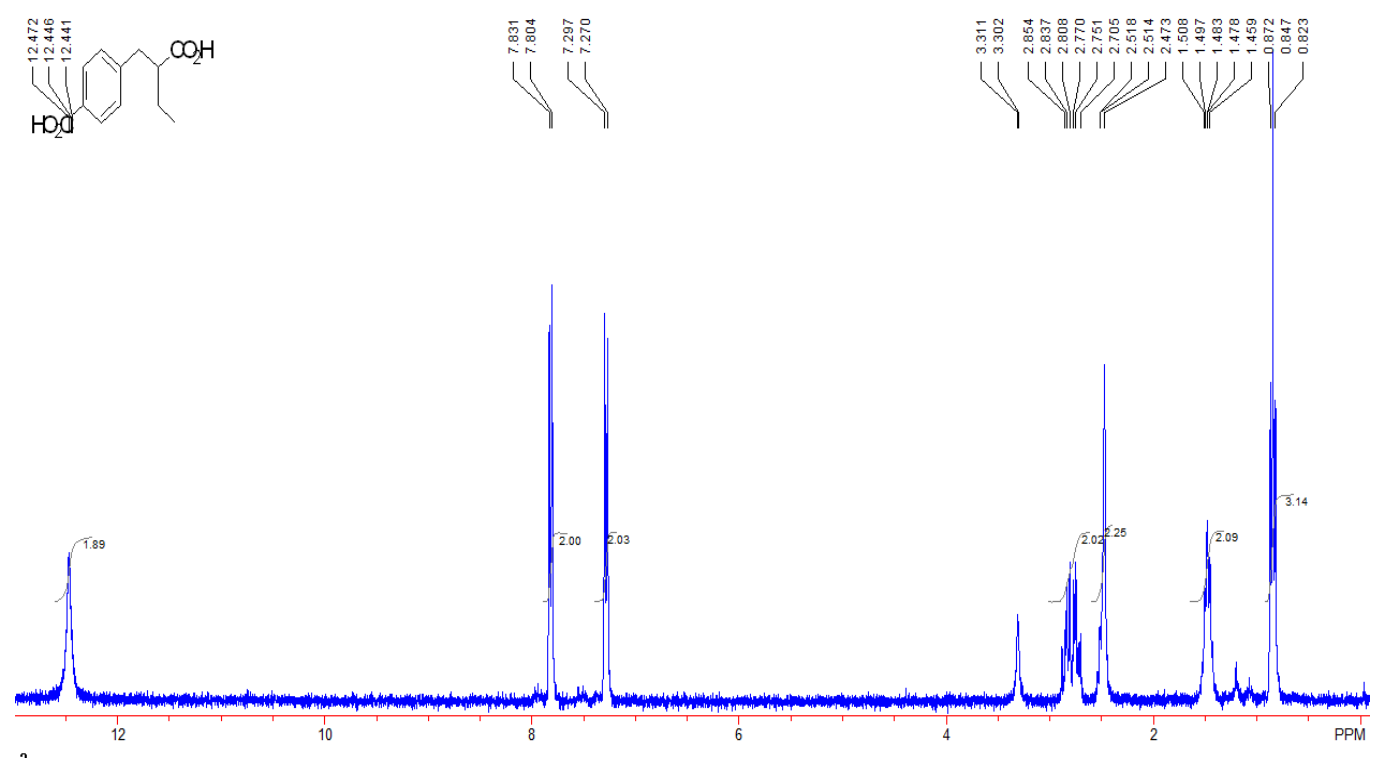

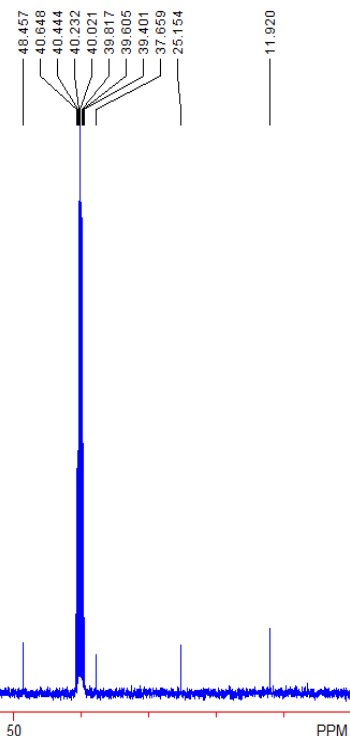



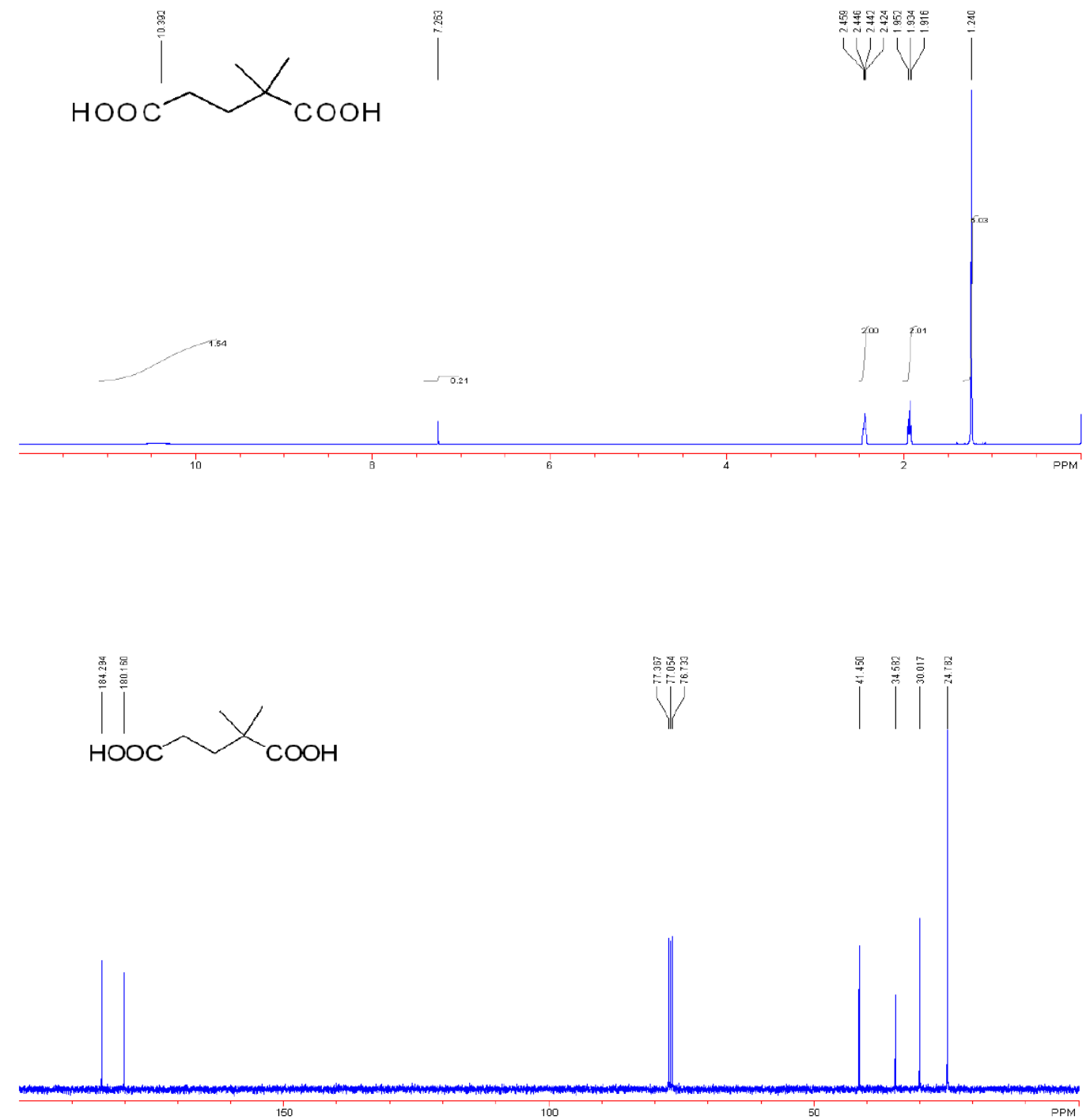
14

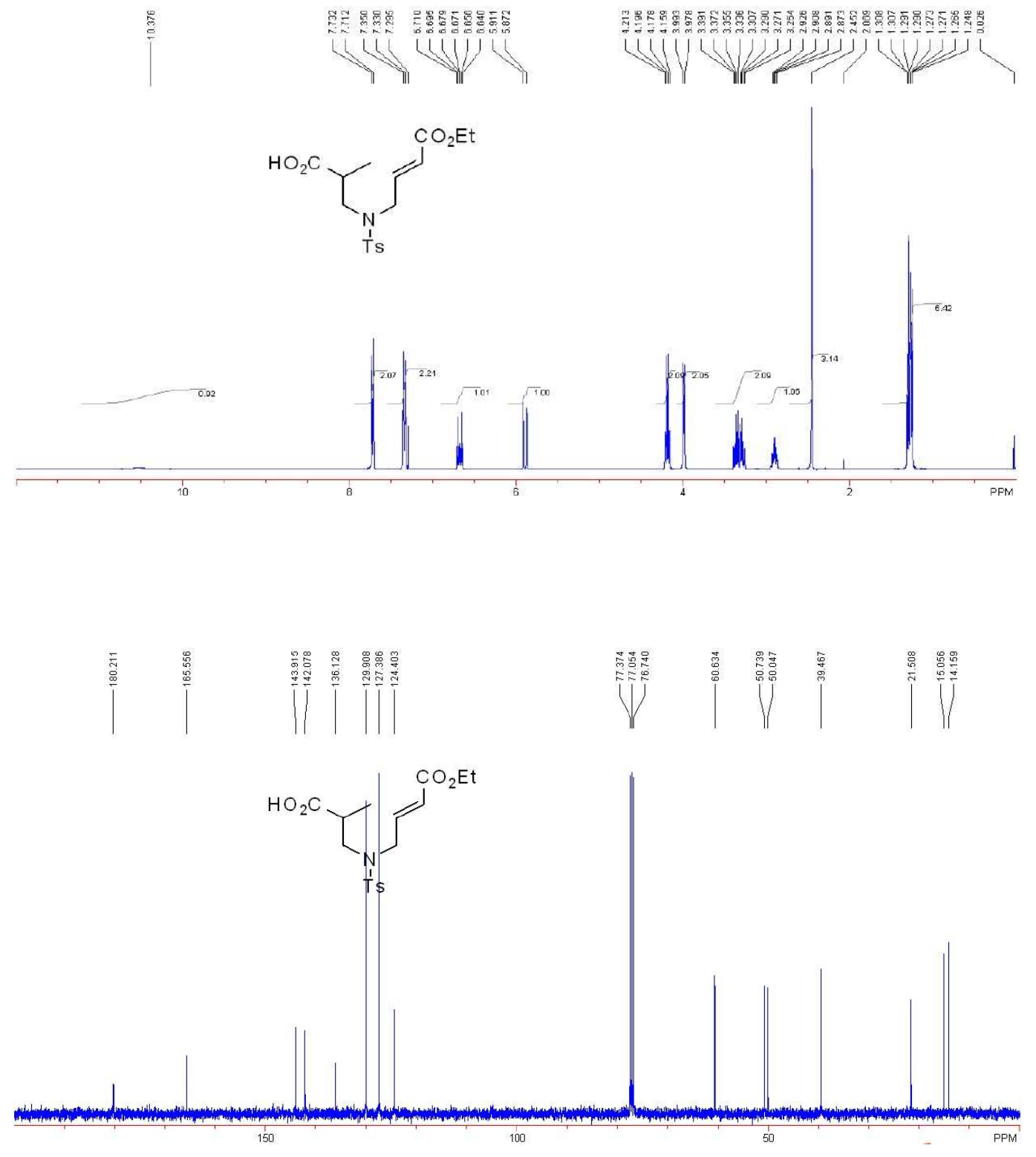


6. ${ }^{1} \mathrm{H}$ and ${ }^{13} \mathrm{C}$ NMR Spectra of Products

3a
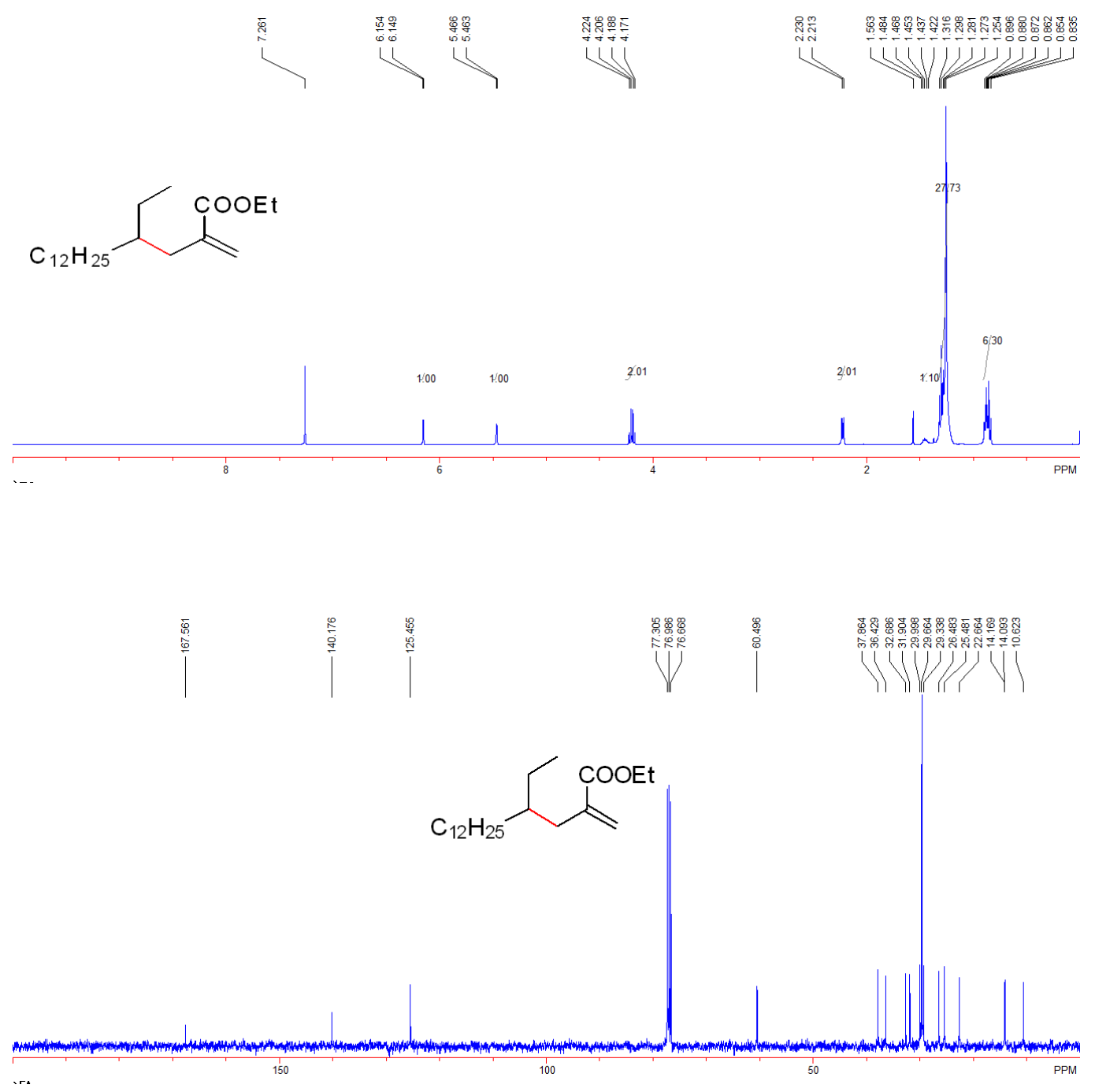
3b

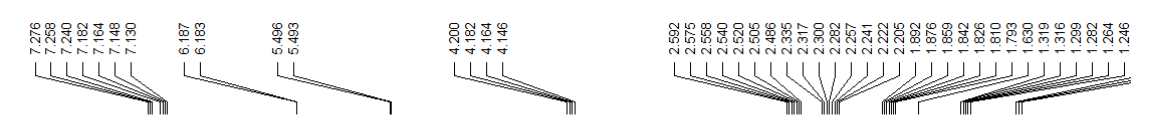

$\rightarrow>c$ coot
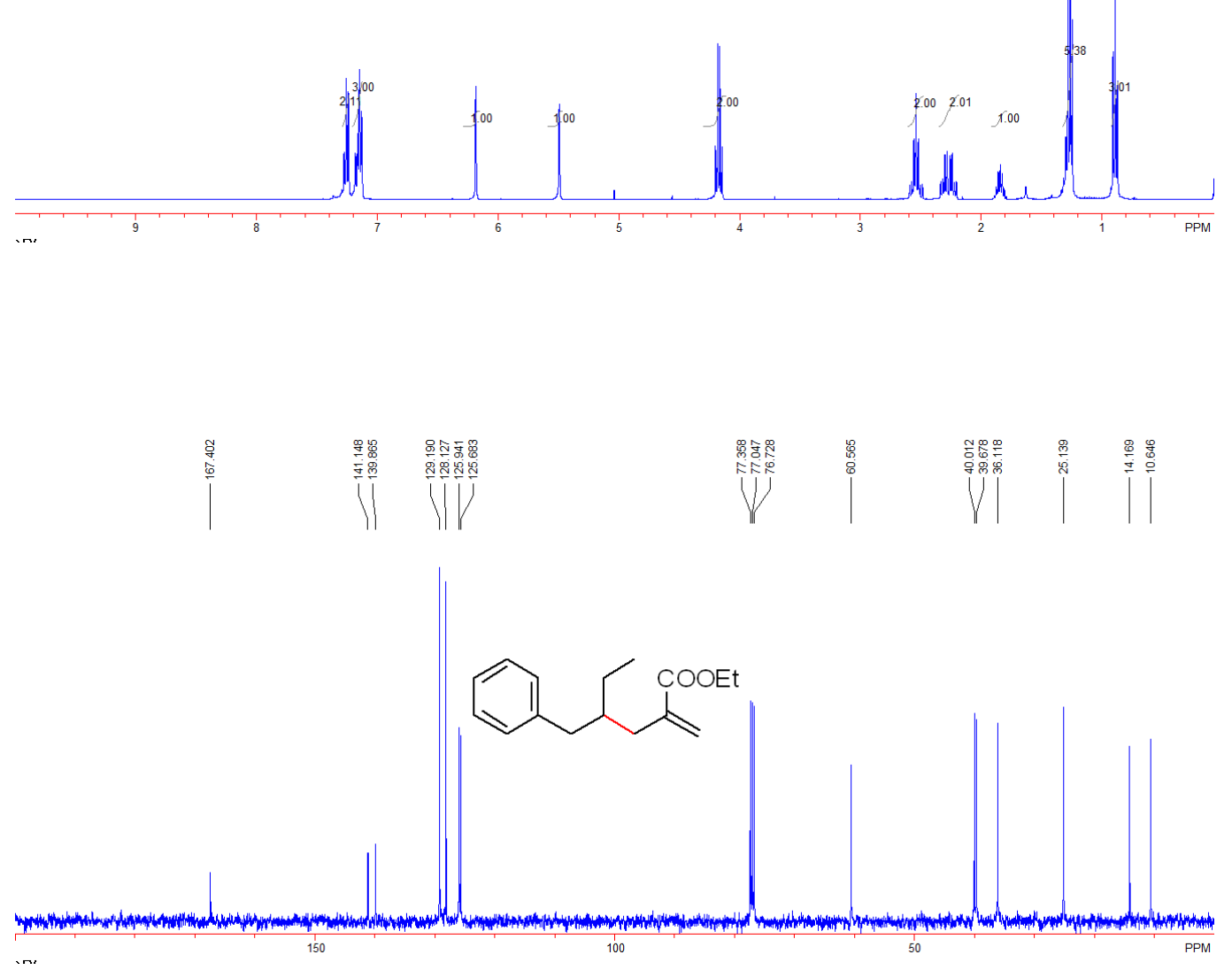
3c
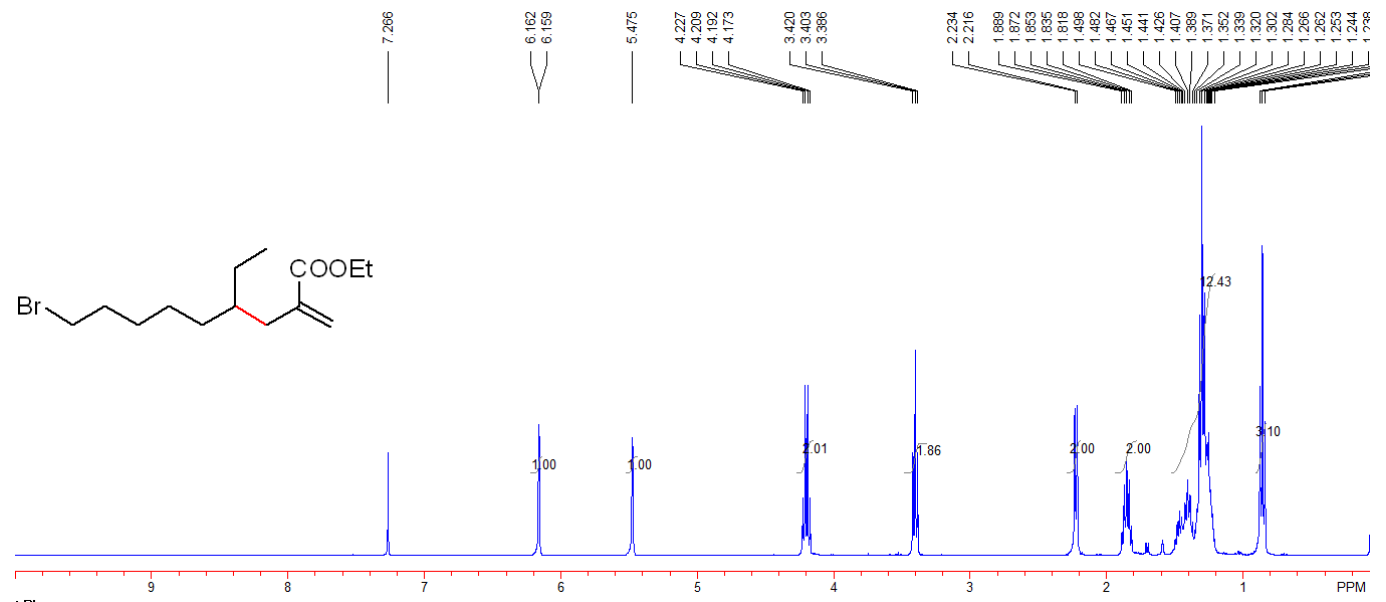

m
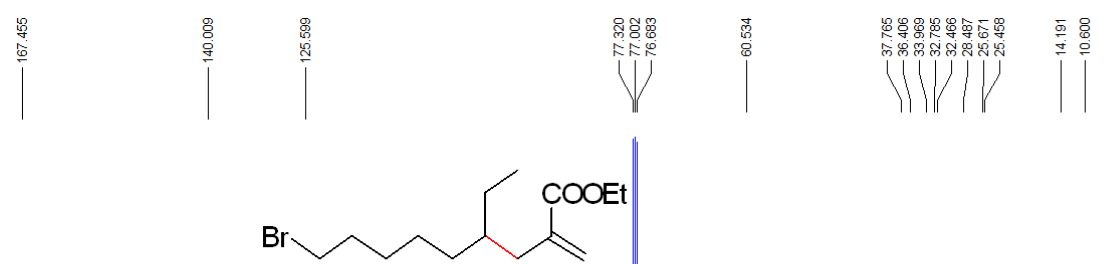

.n 
3d
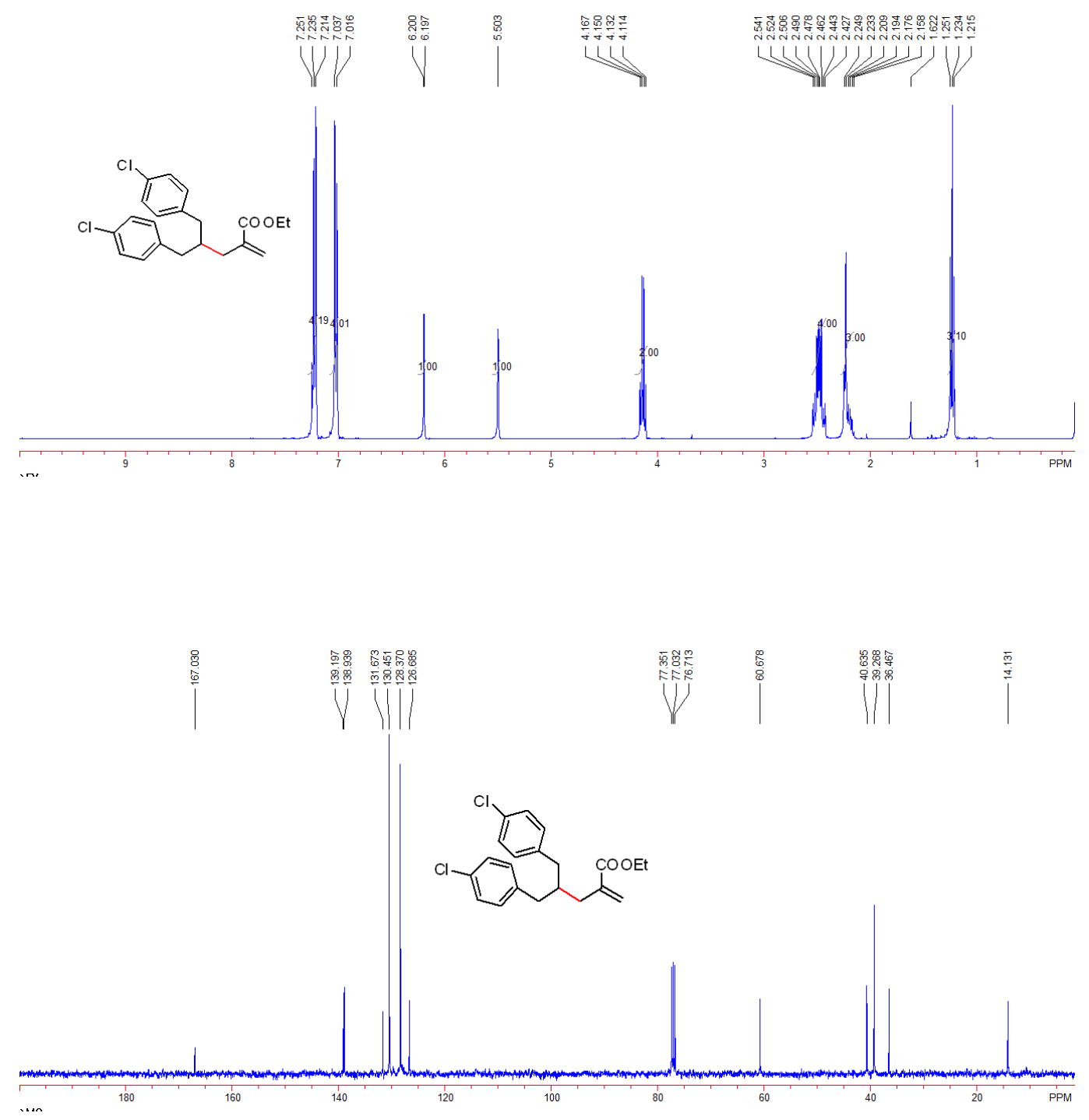


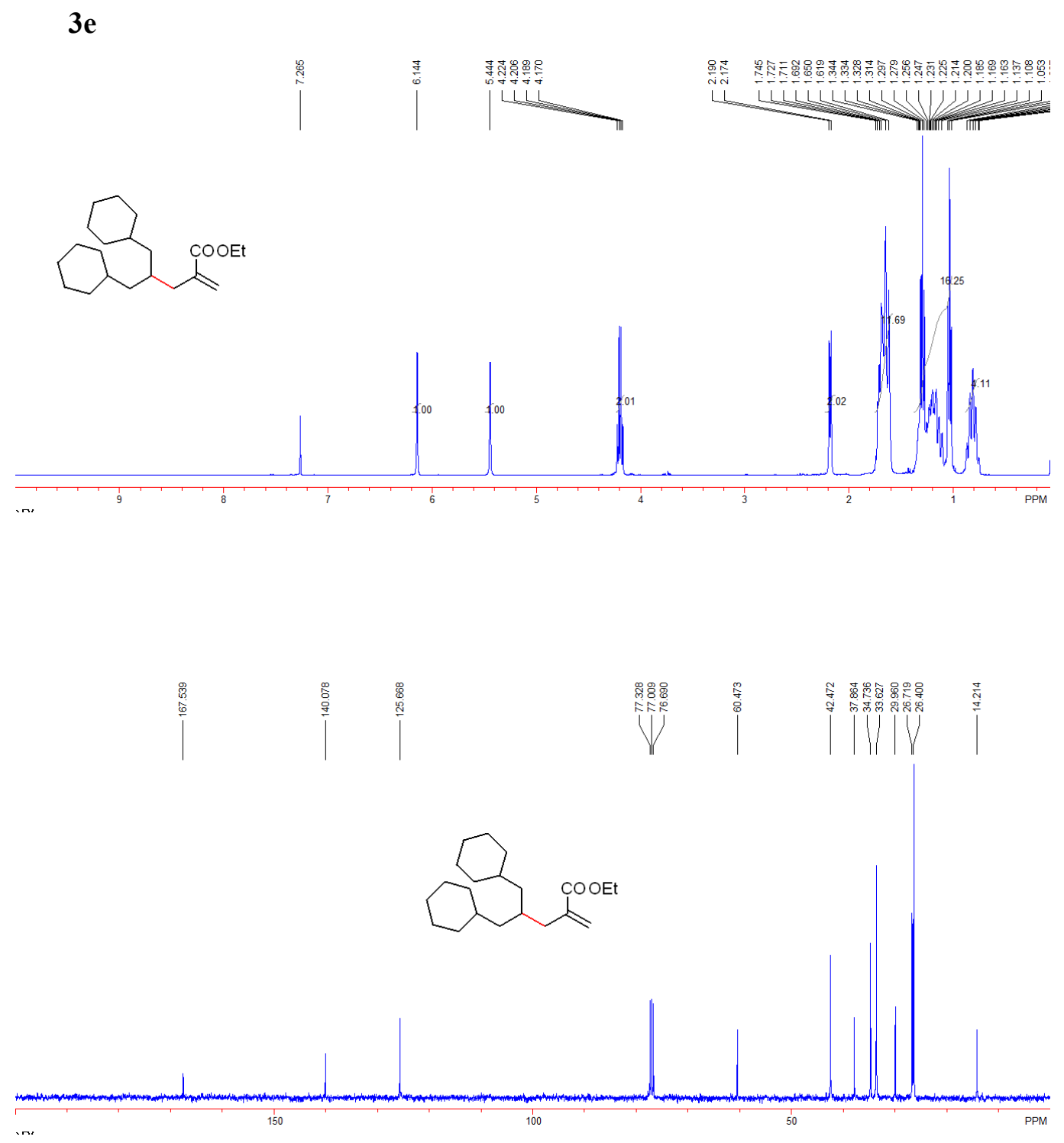


3f

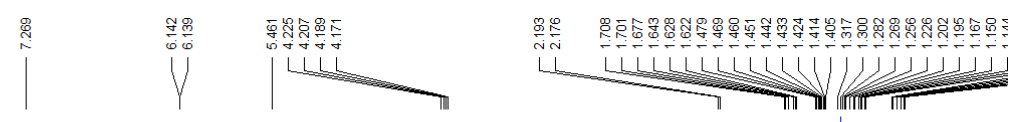

$\longrightarrow \mathrm{COOEt}^{\mathrm{CO}}$

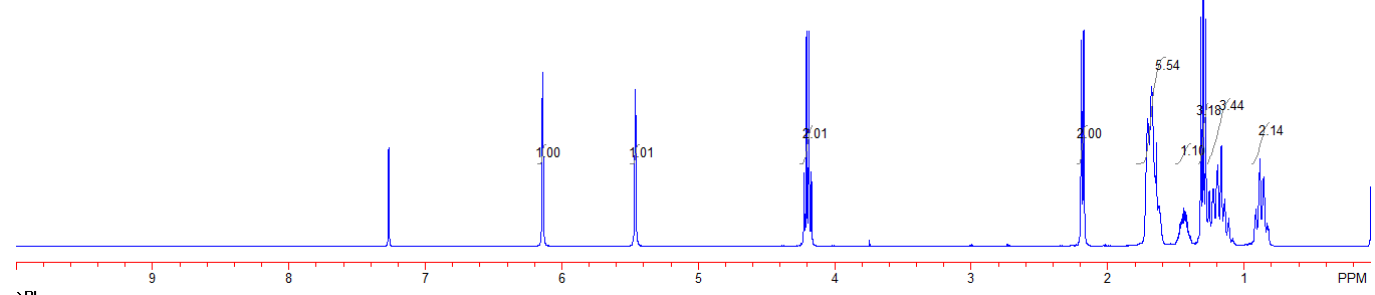

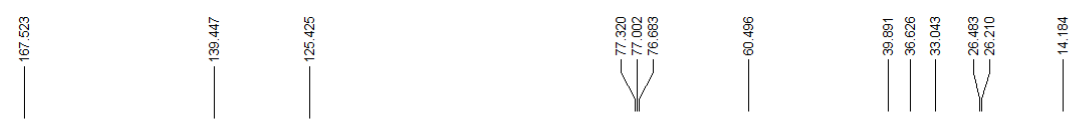

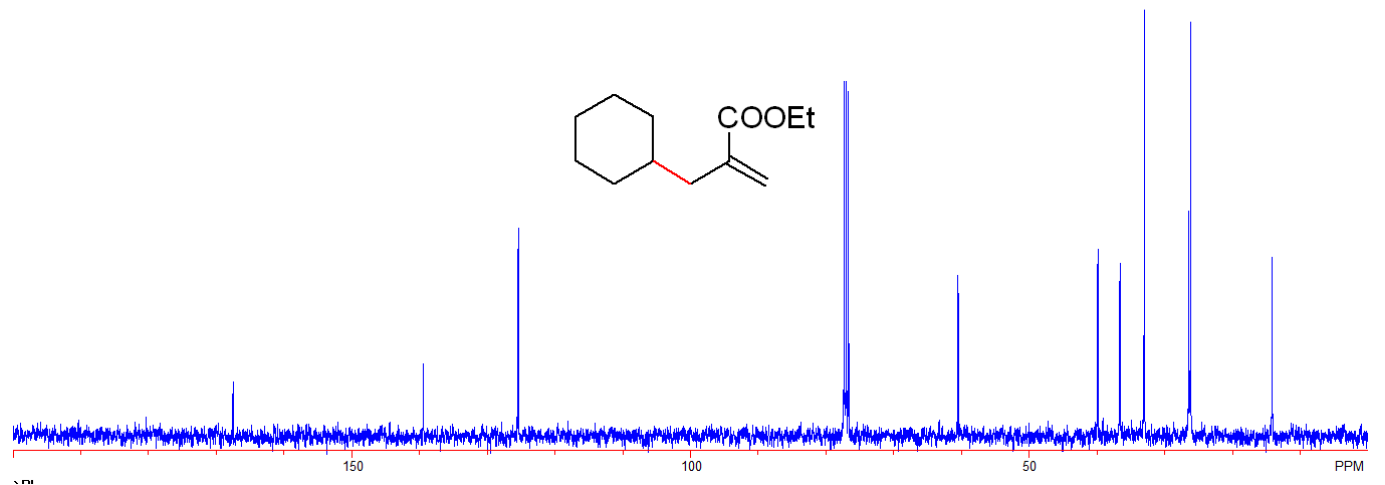

.n. 
$3 g$

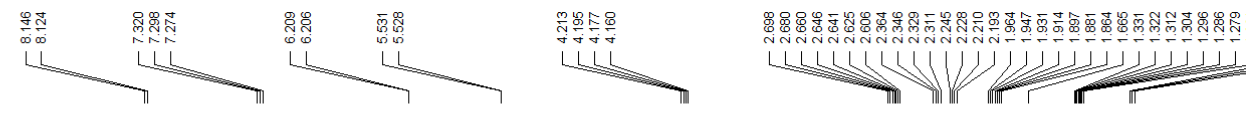

$\mathrm{O}_{2} \mathrm{~N}$

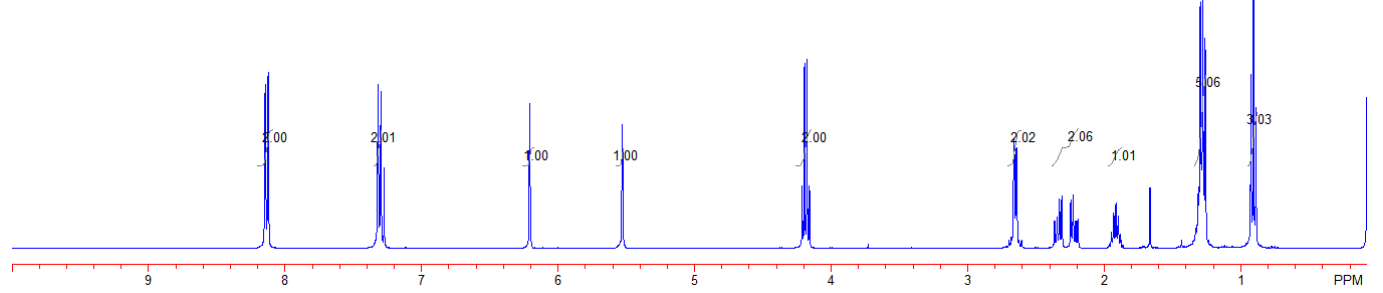

--

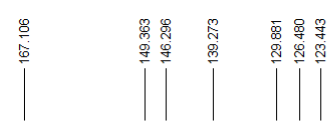
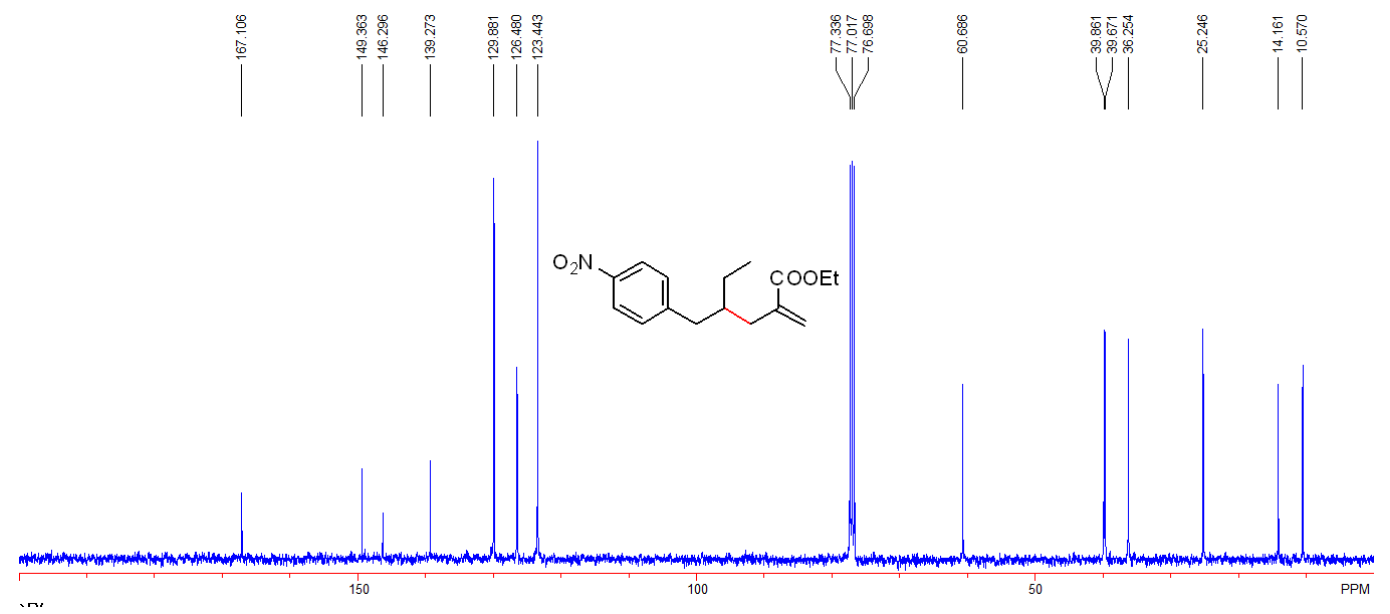
3h
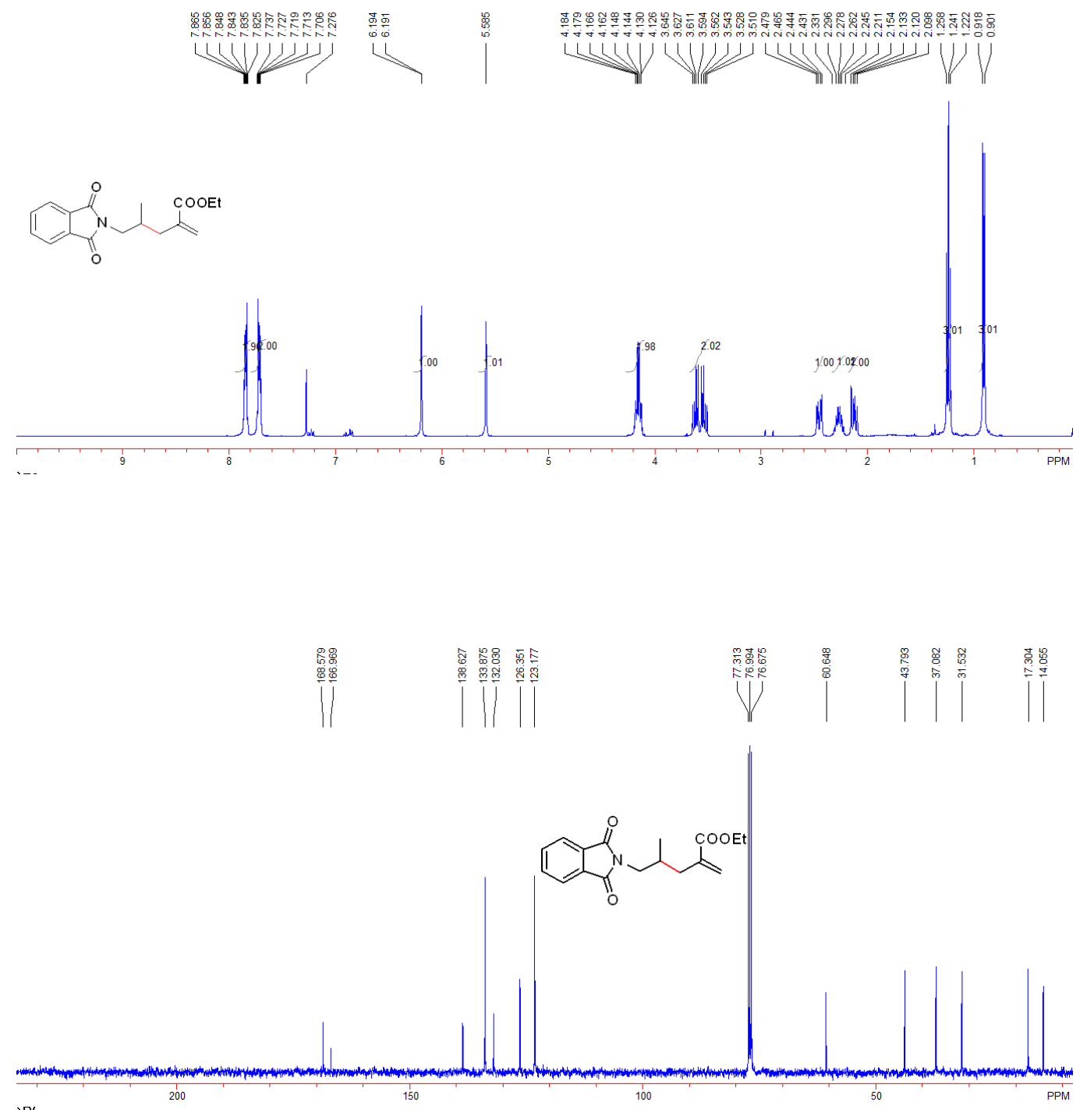
$3 i$
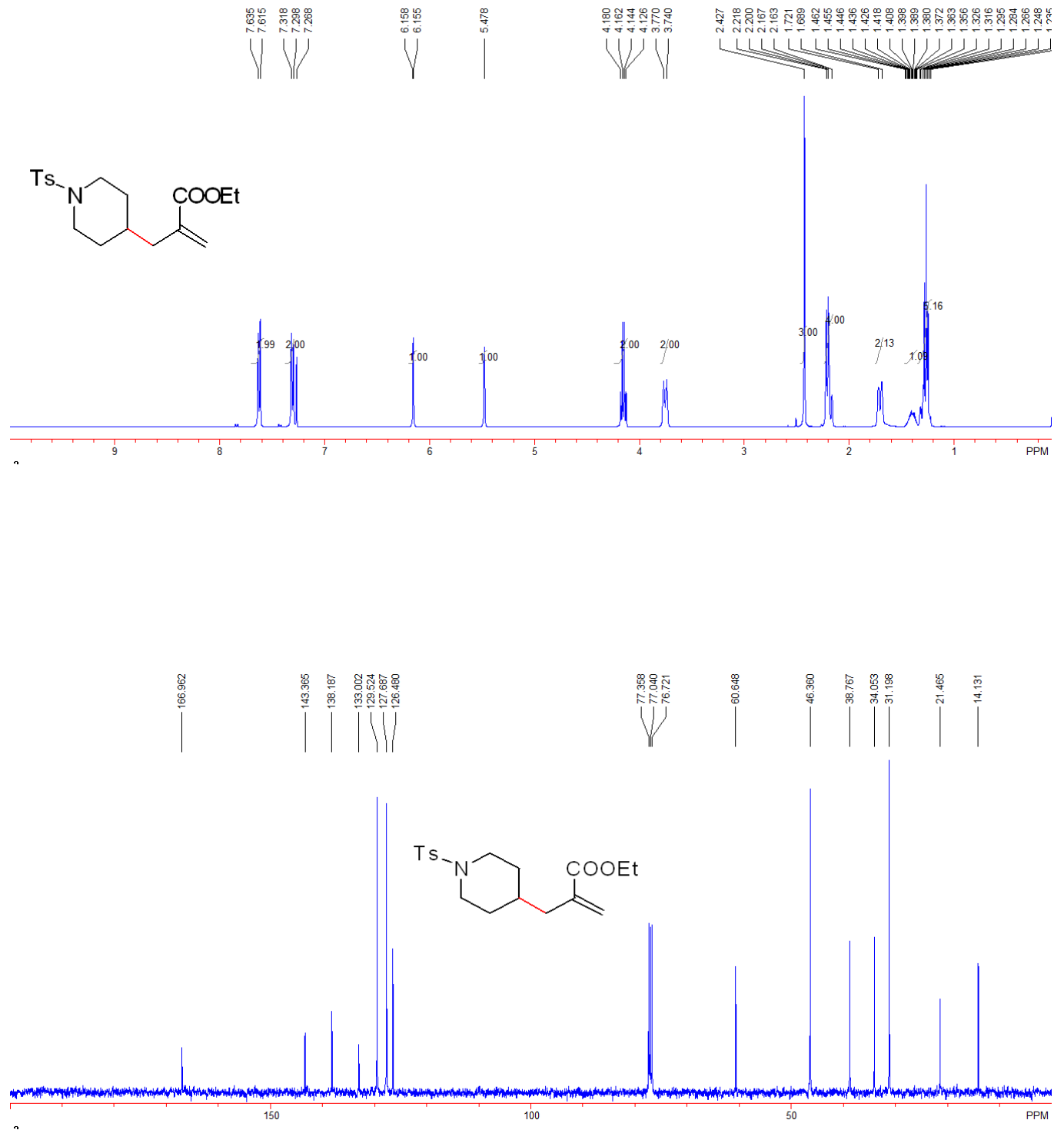
3j
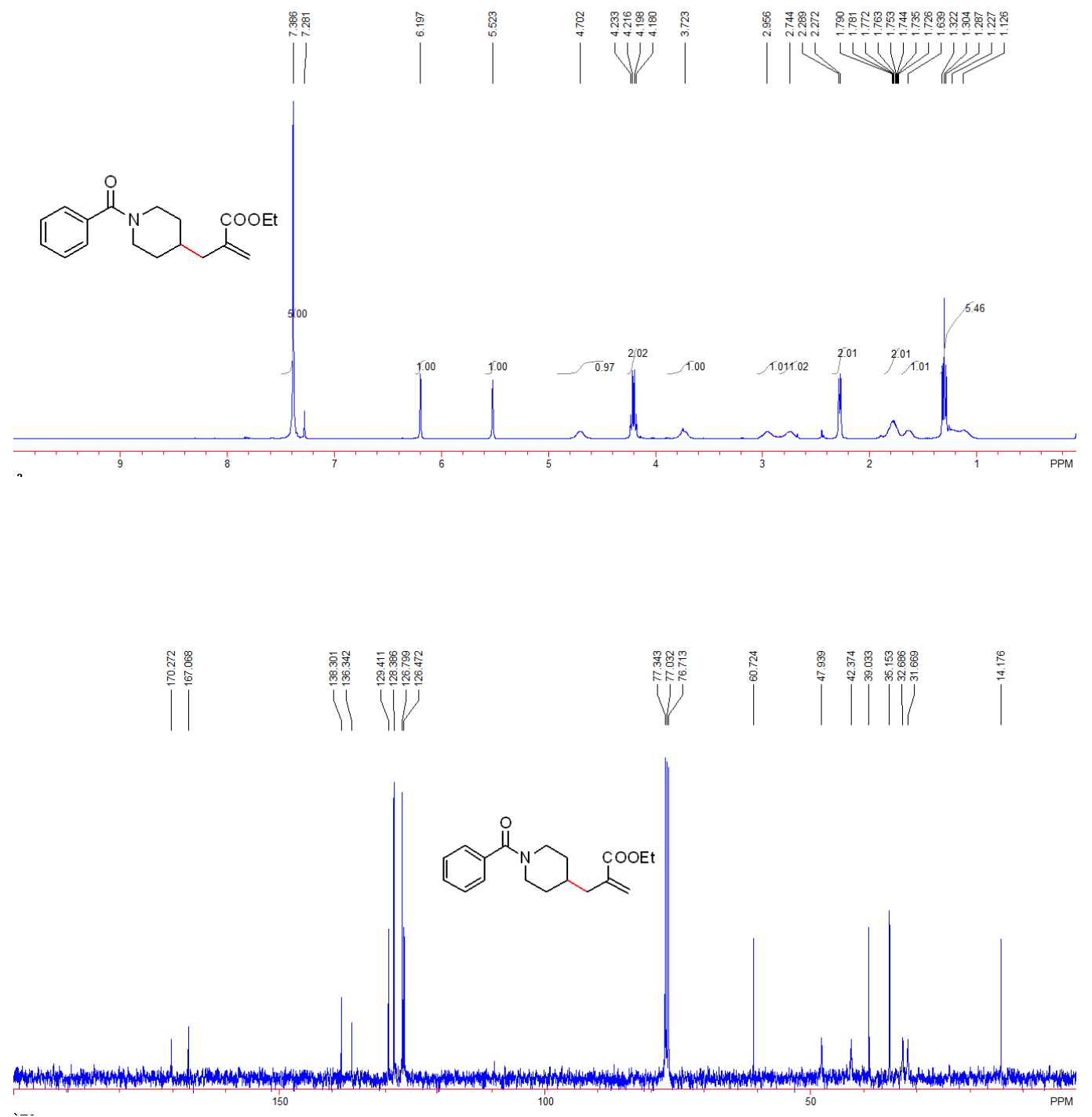
3k
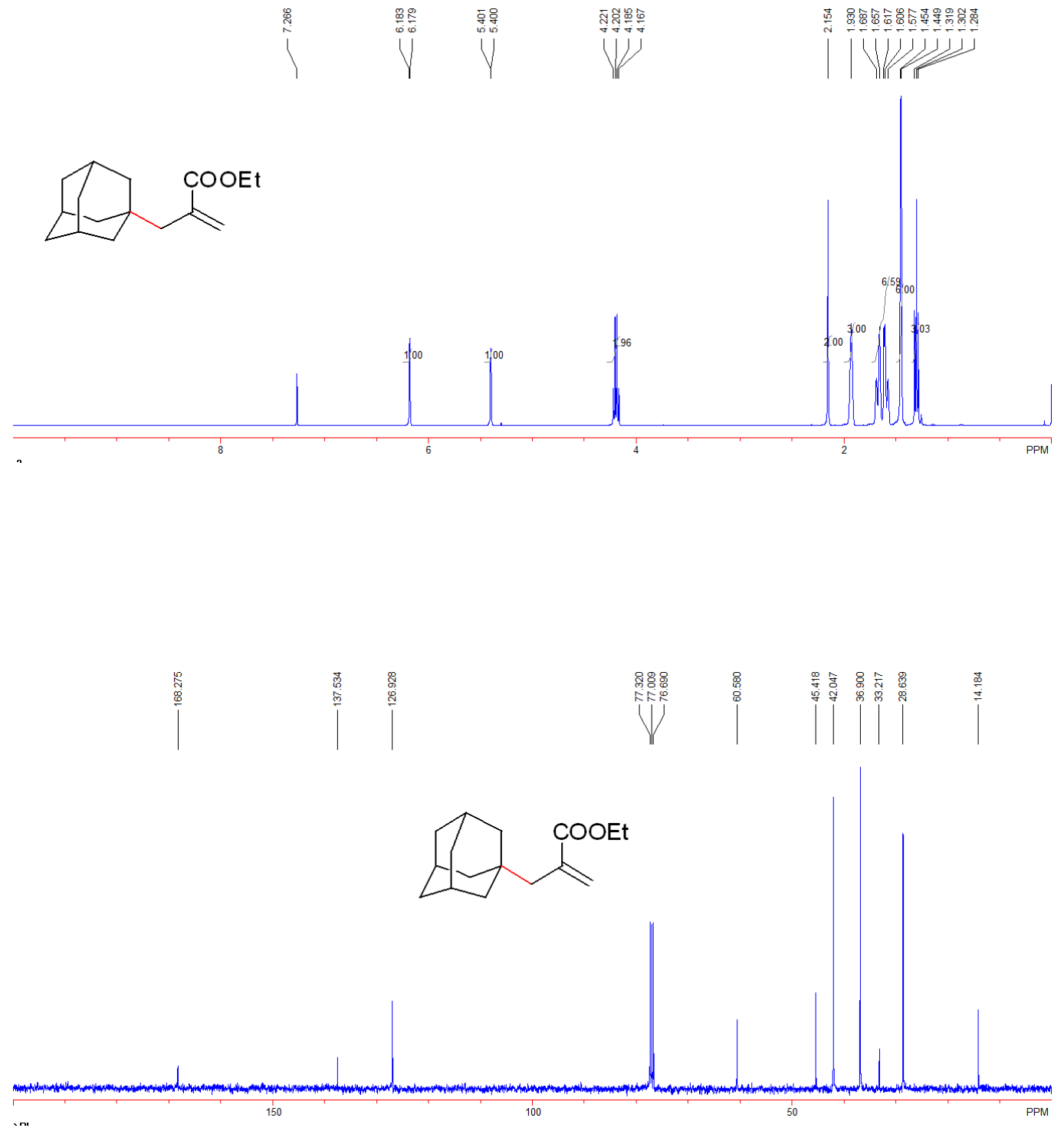
31

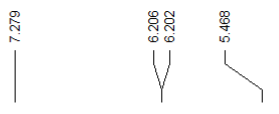

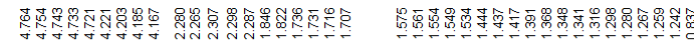

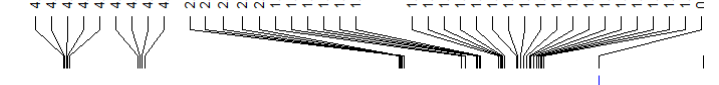

$\sigma_{0}^{O} x^{\text {COOEt }}$
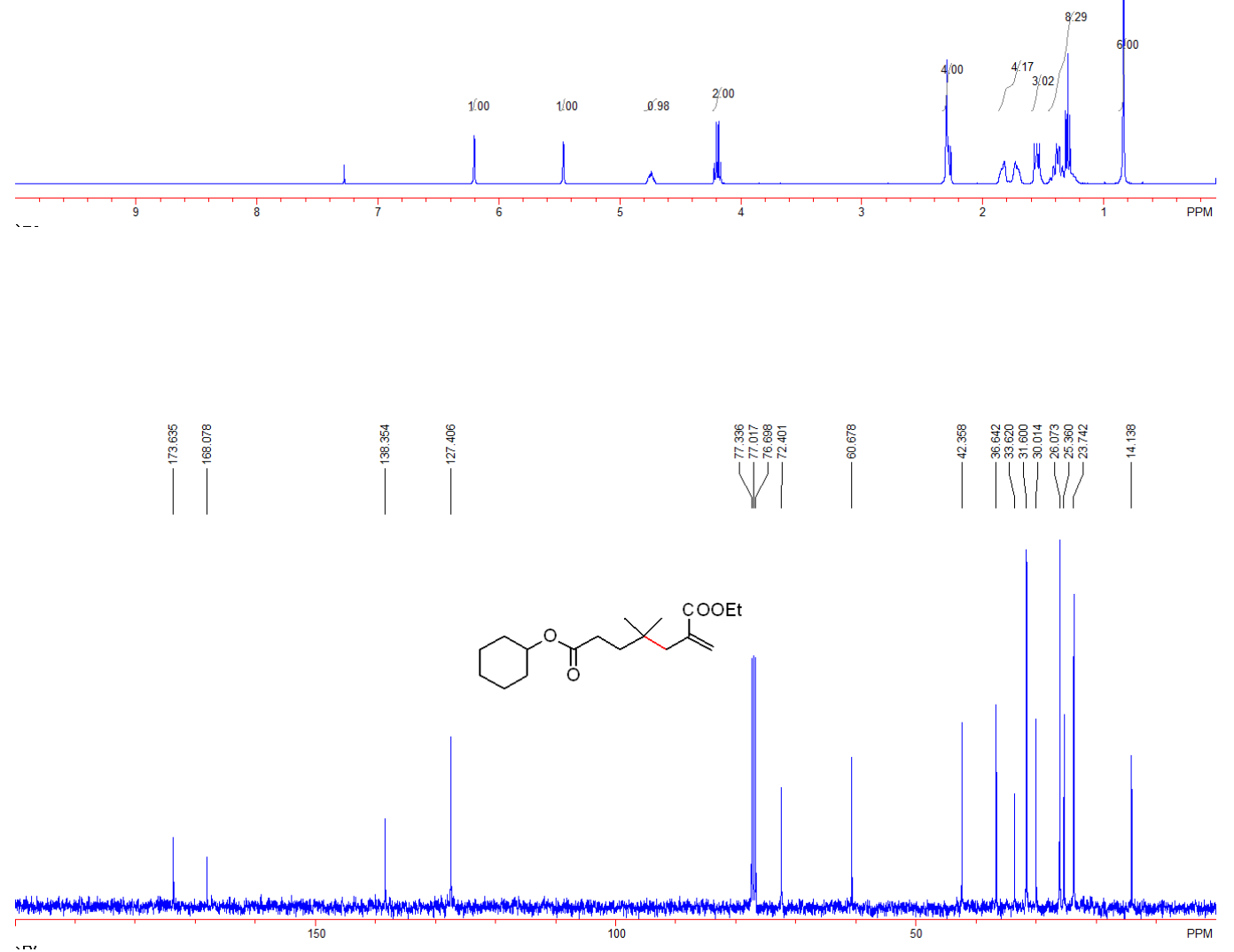
$3 \mathrm{~m}$
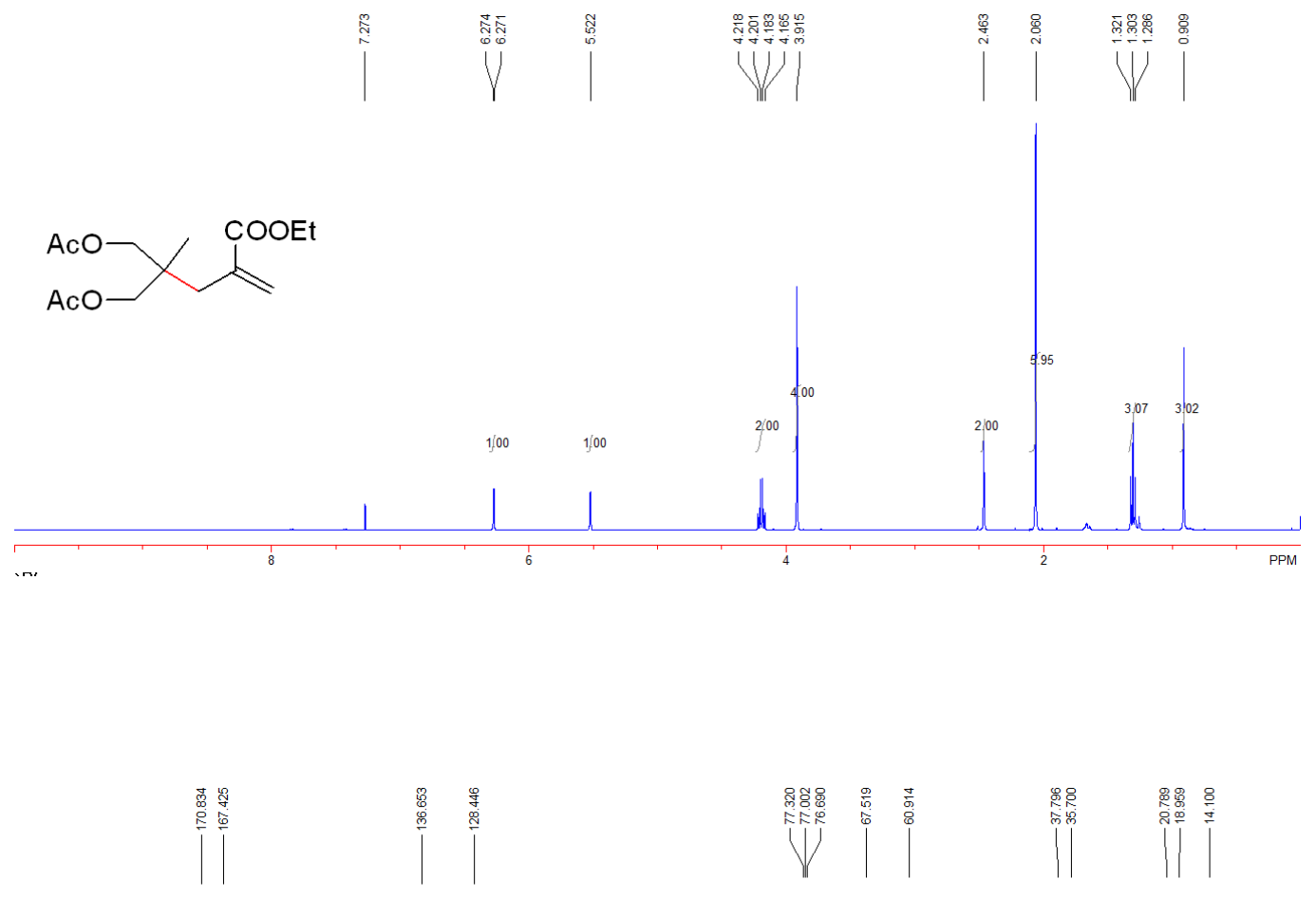

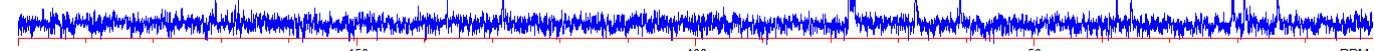


3n
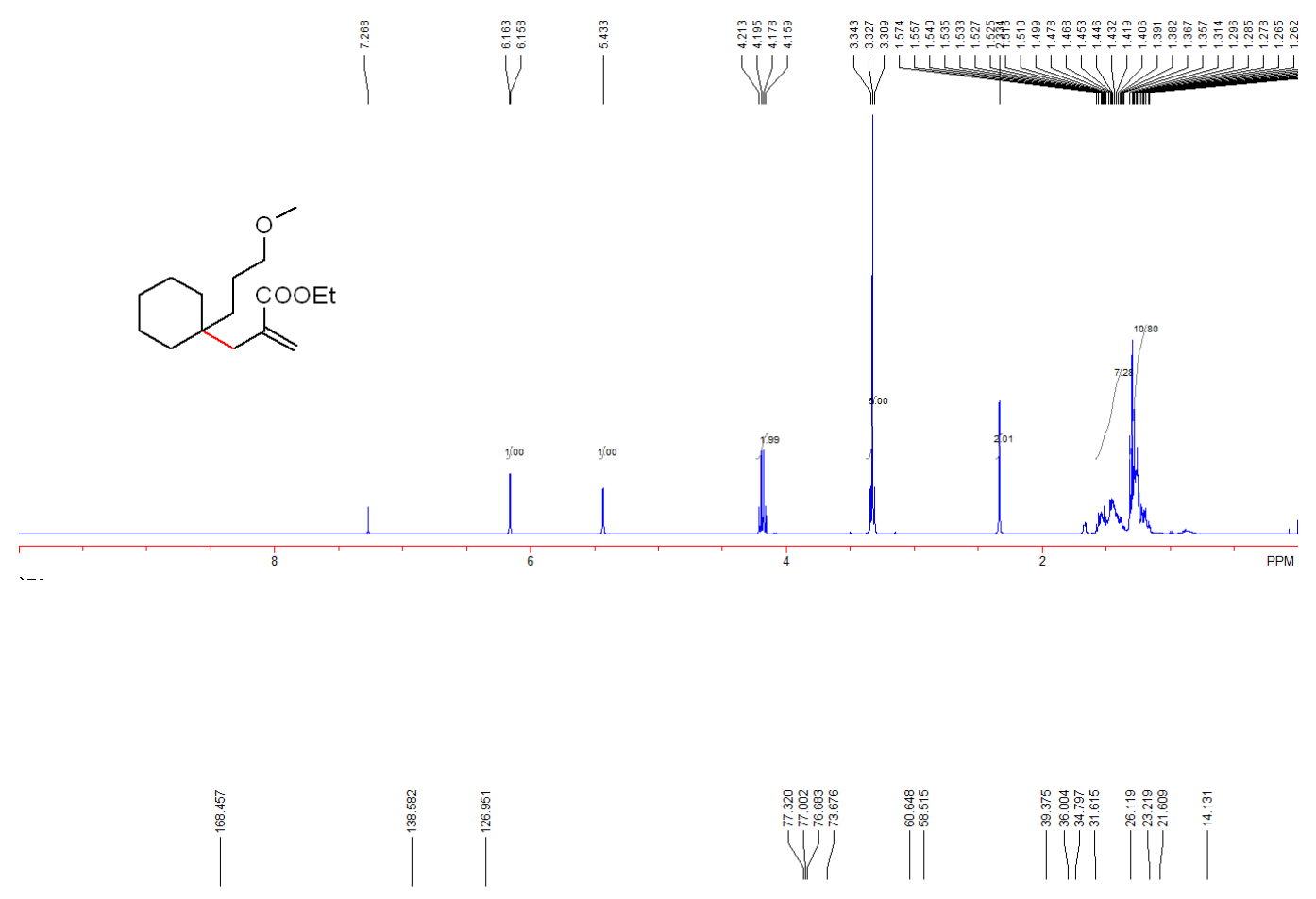

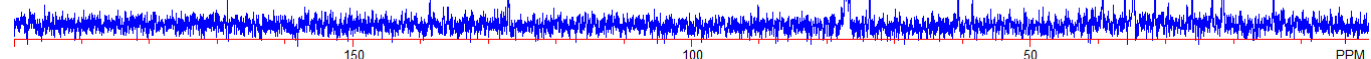

.... 

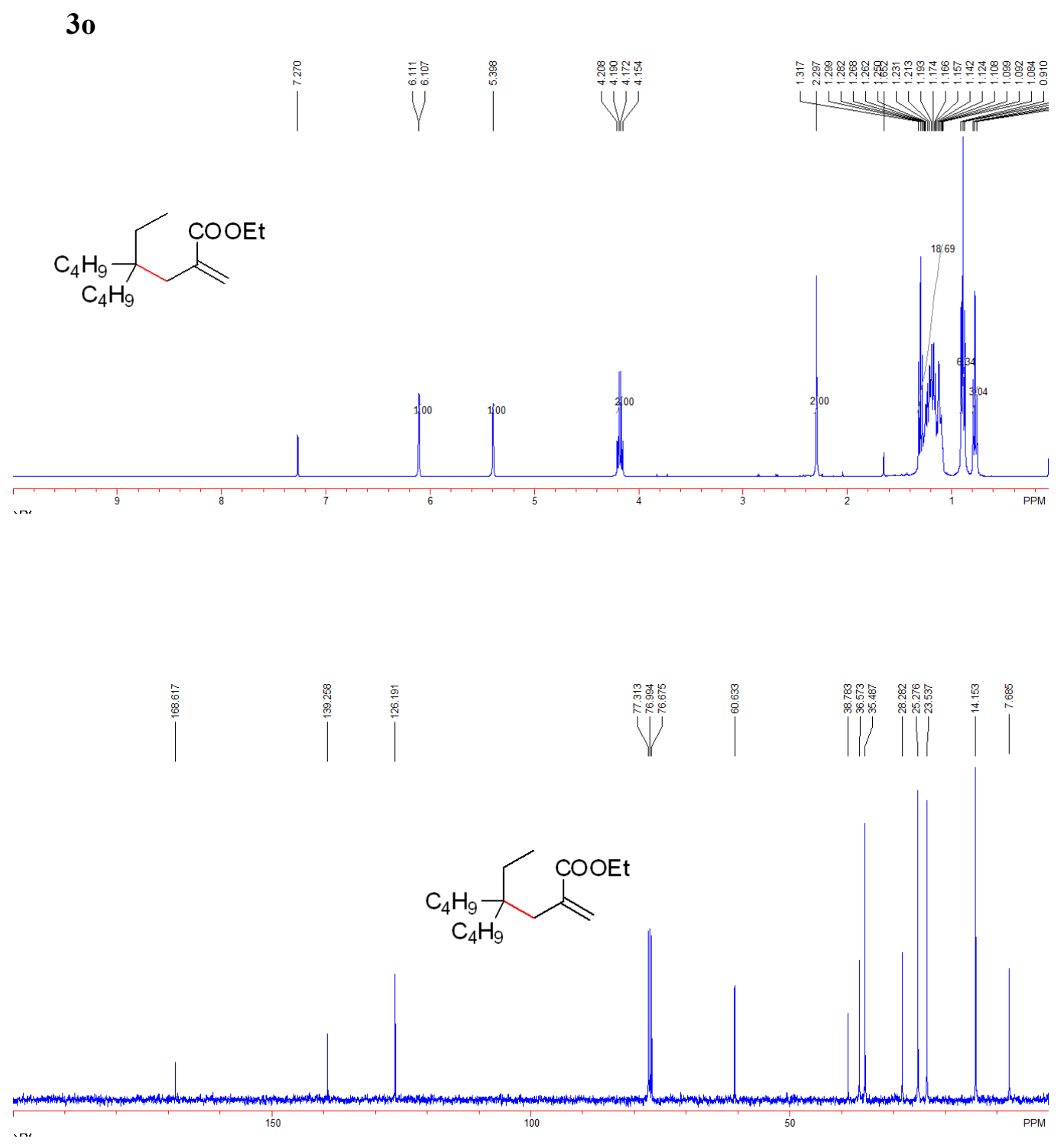
$3 p$
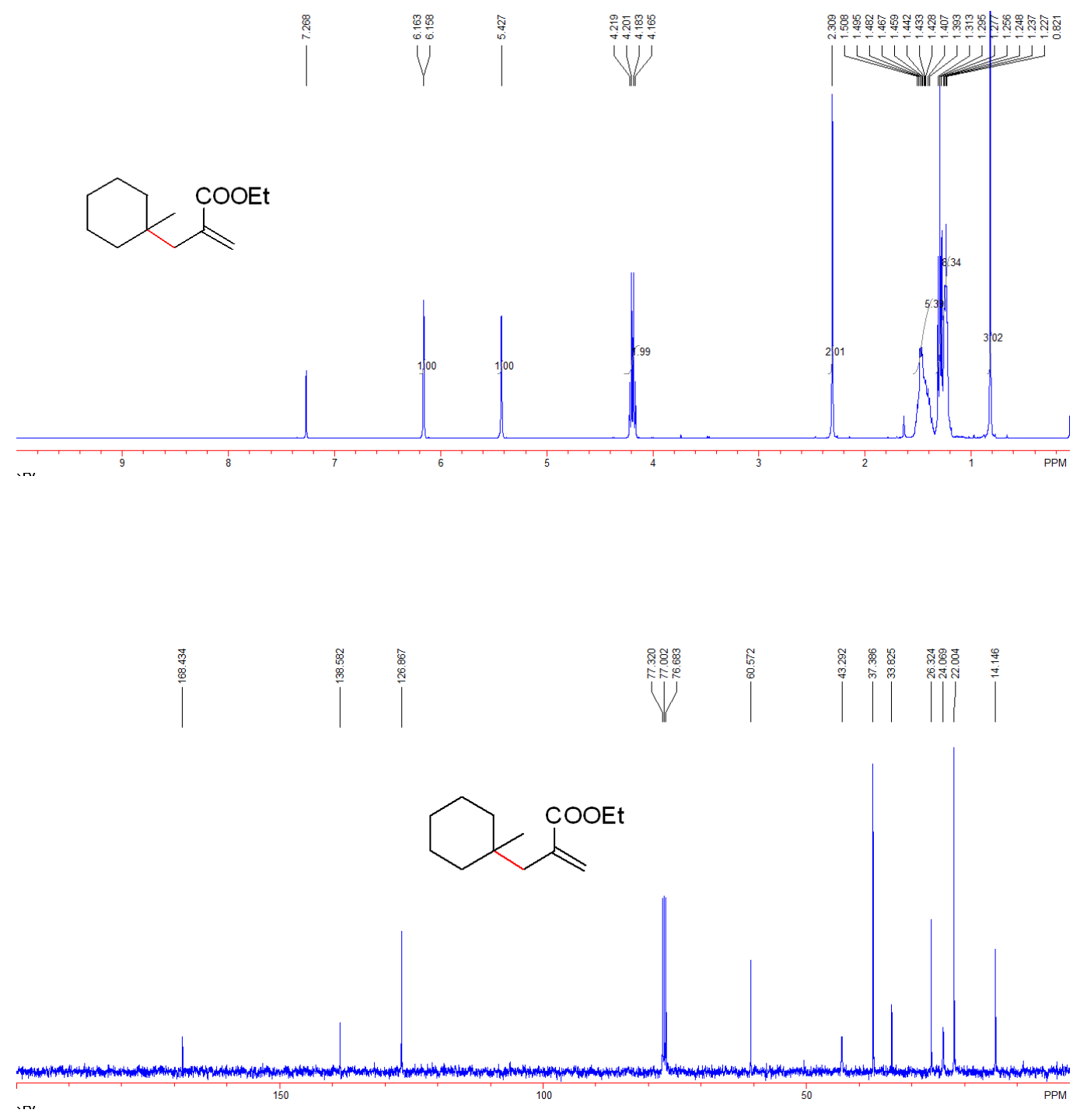

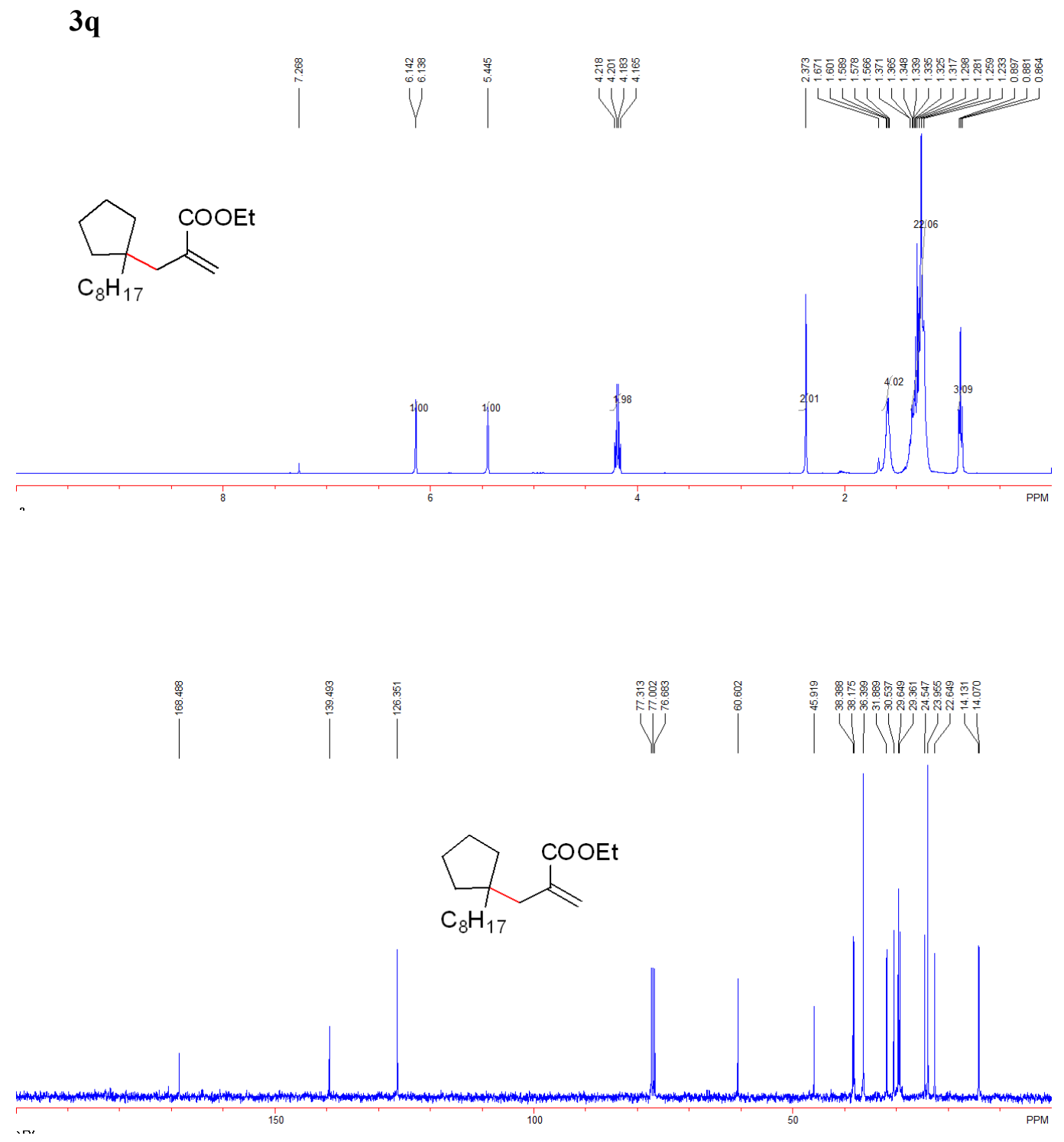

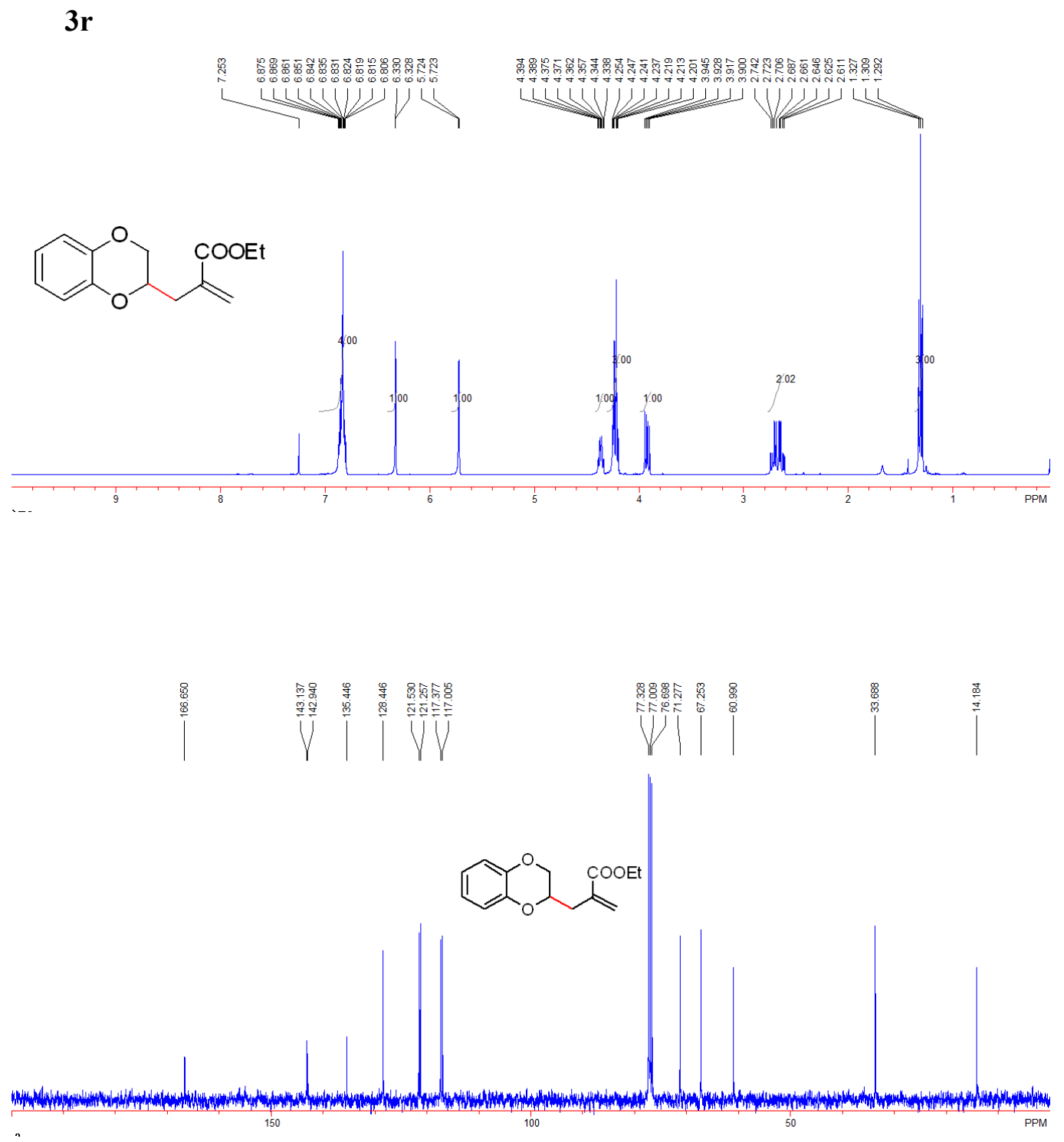
$3 s$

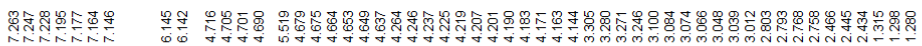

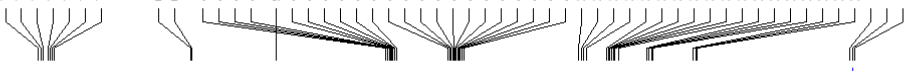
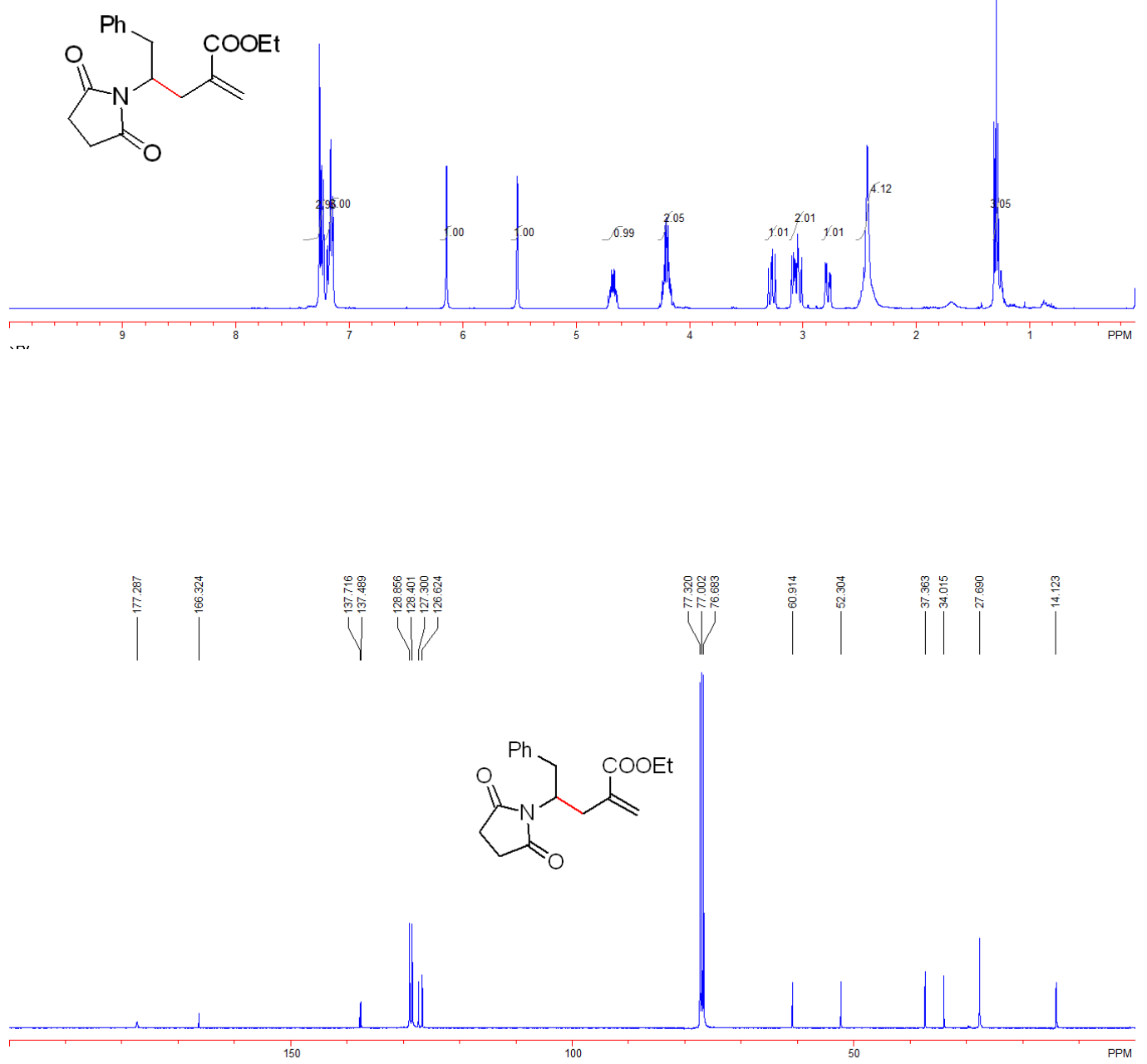

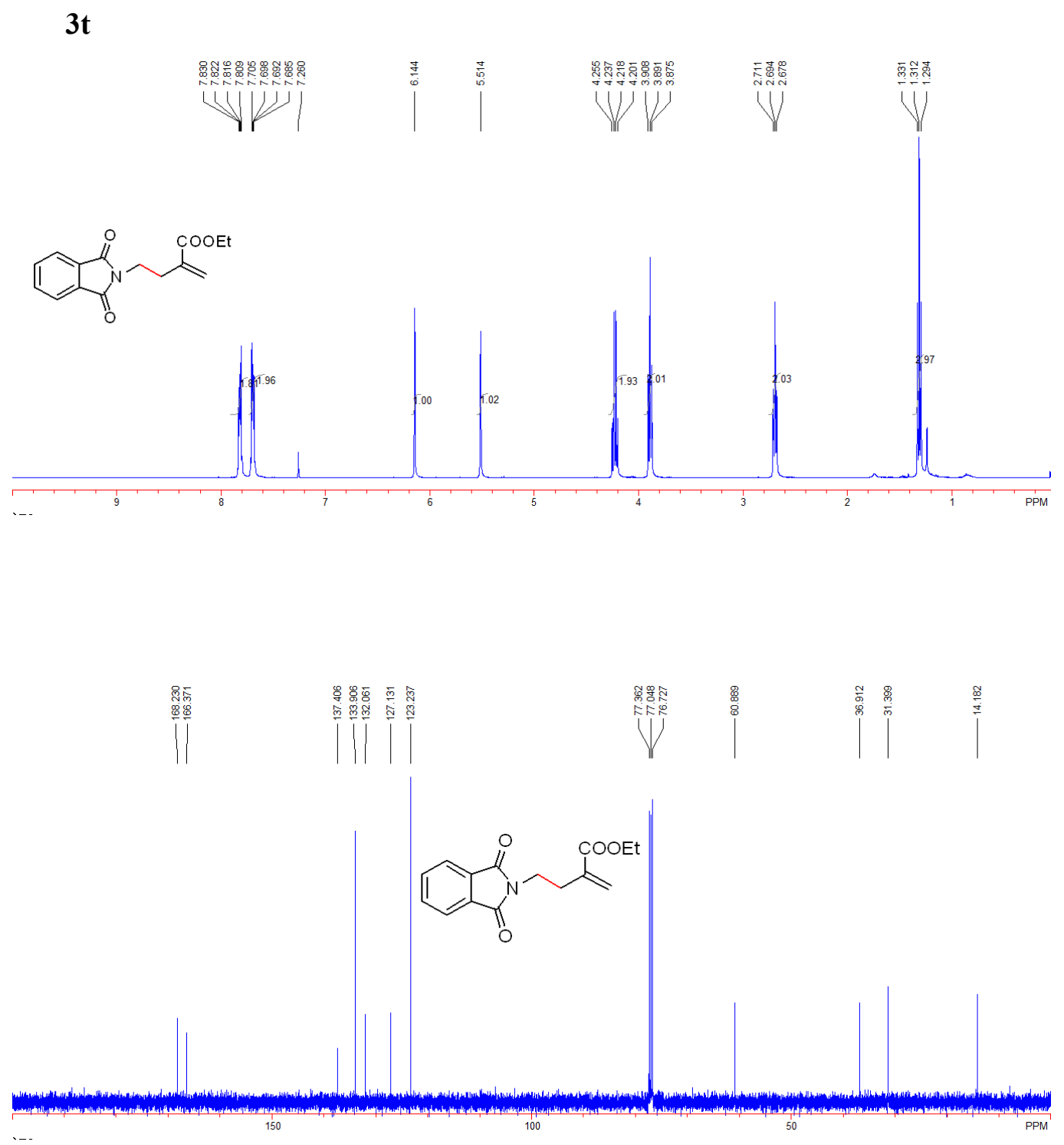
3u
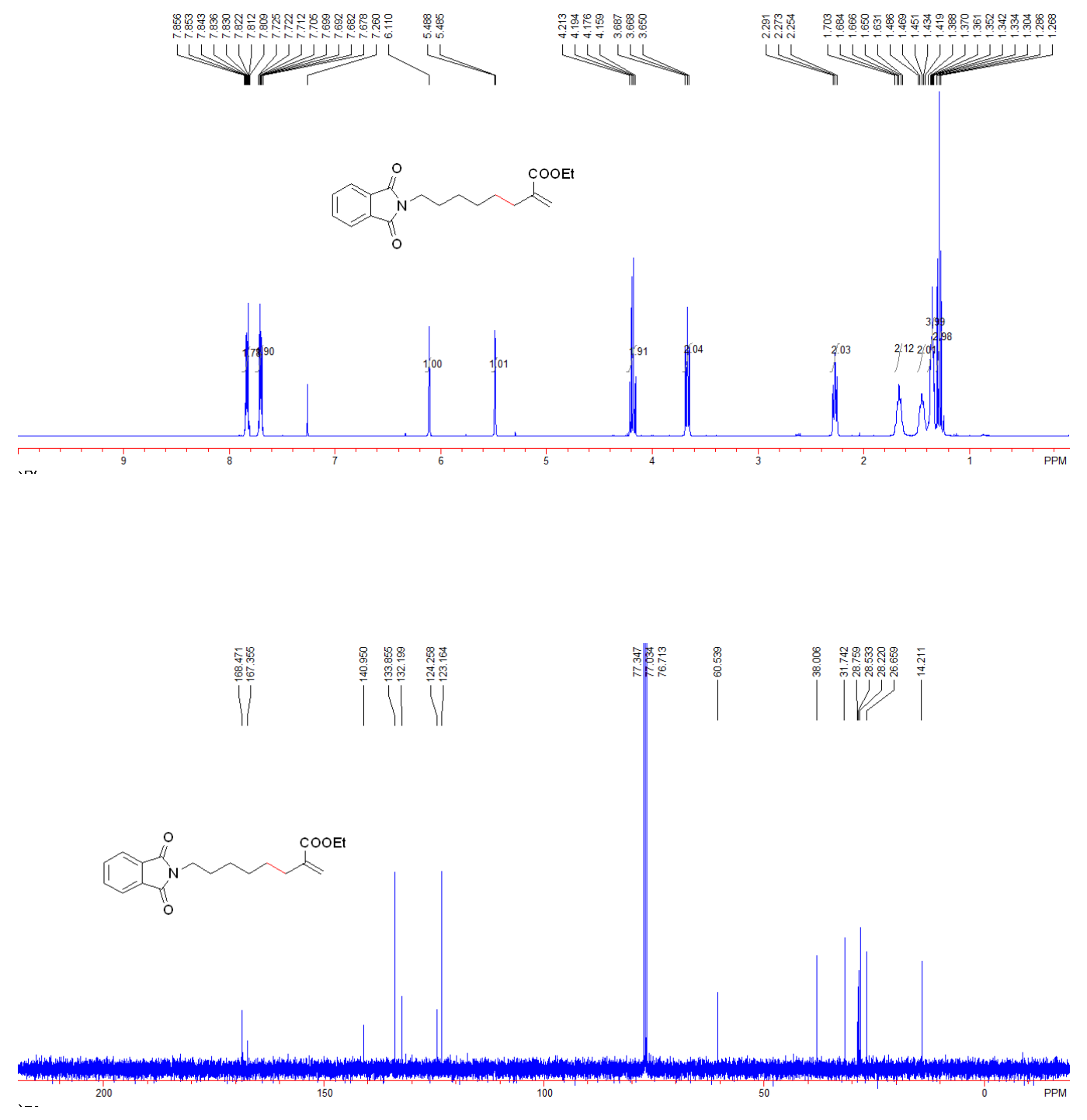


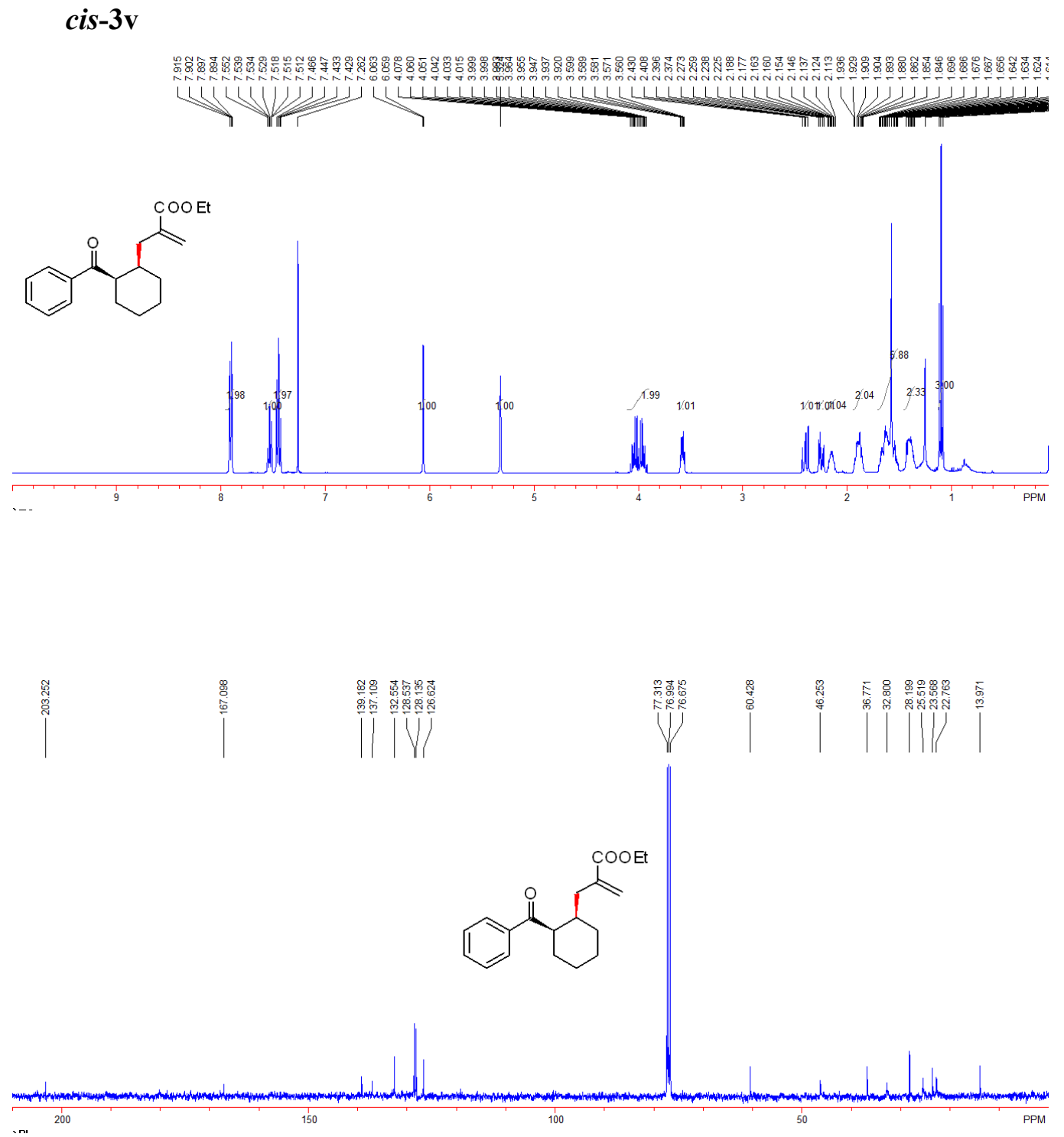


trans-3v
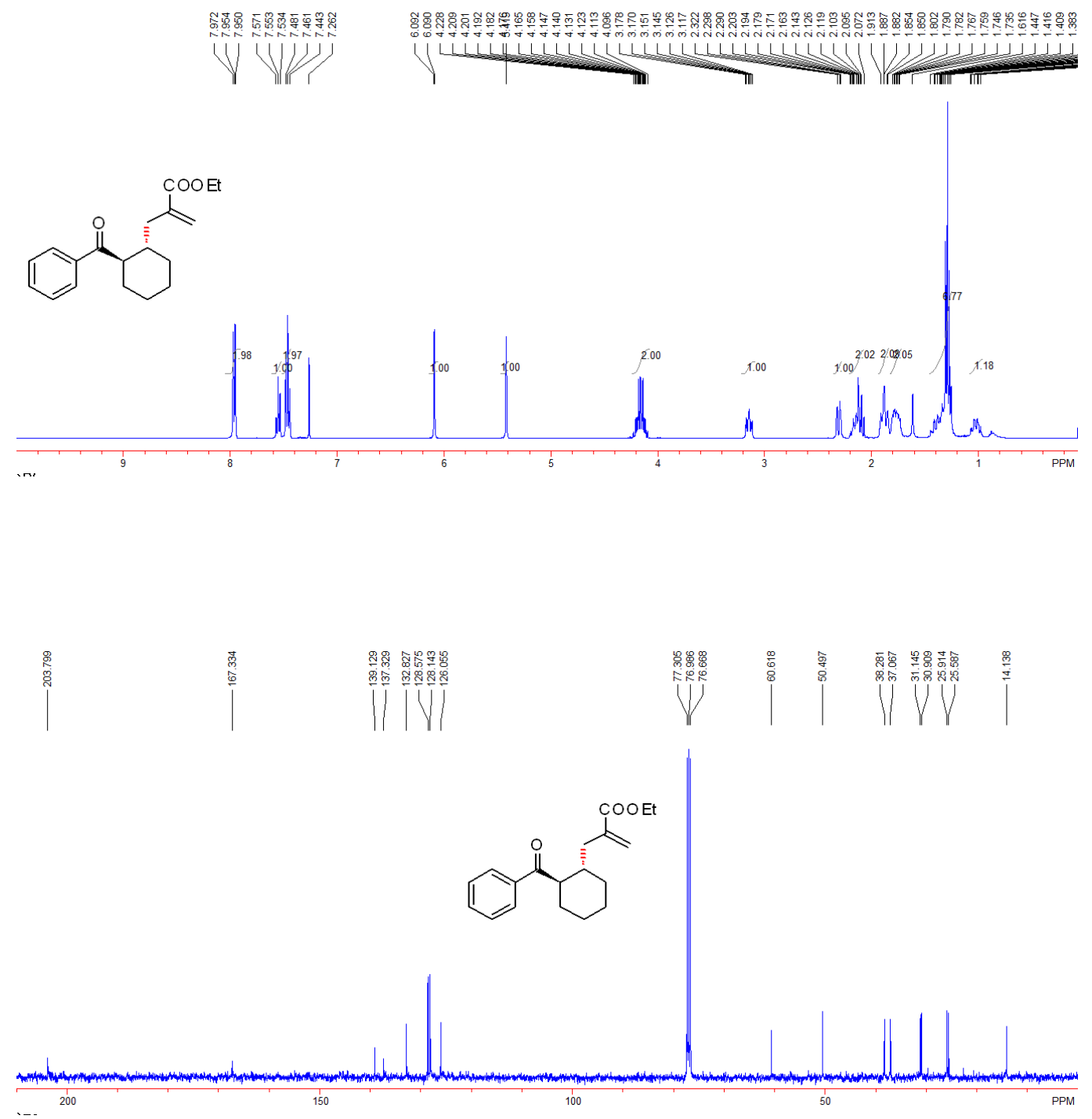


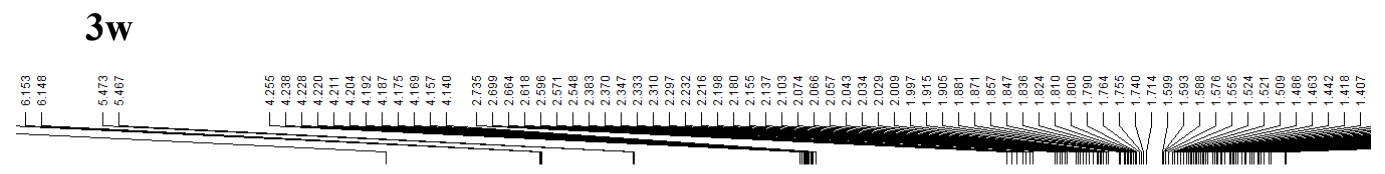

$\overbrace{H}^{C O O E t}$

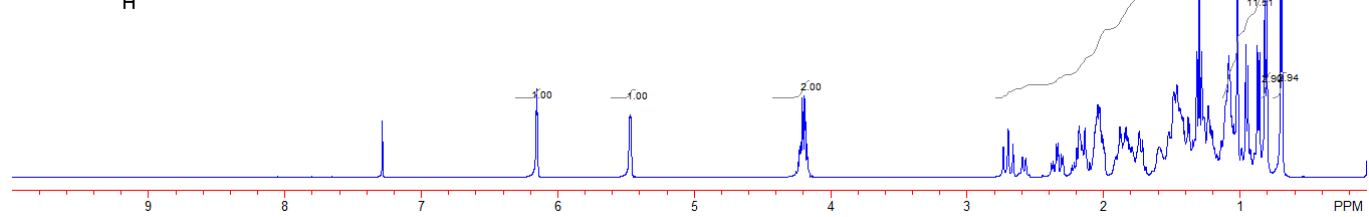

$$
\text { -- }
$$
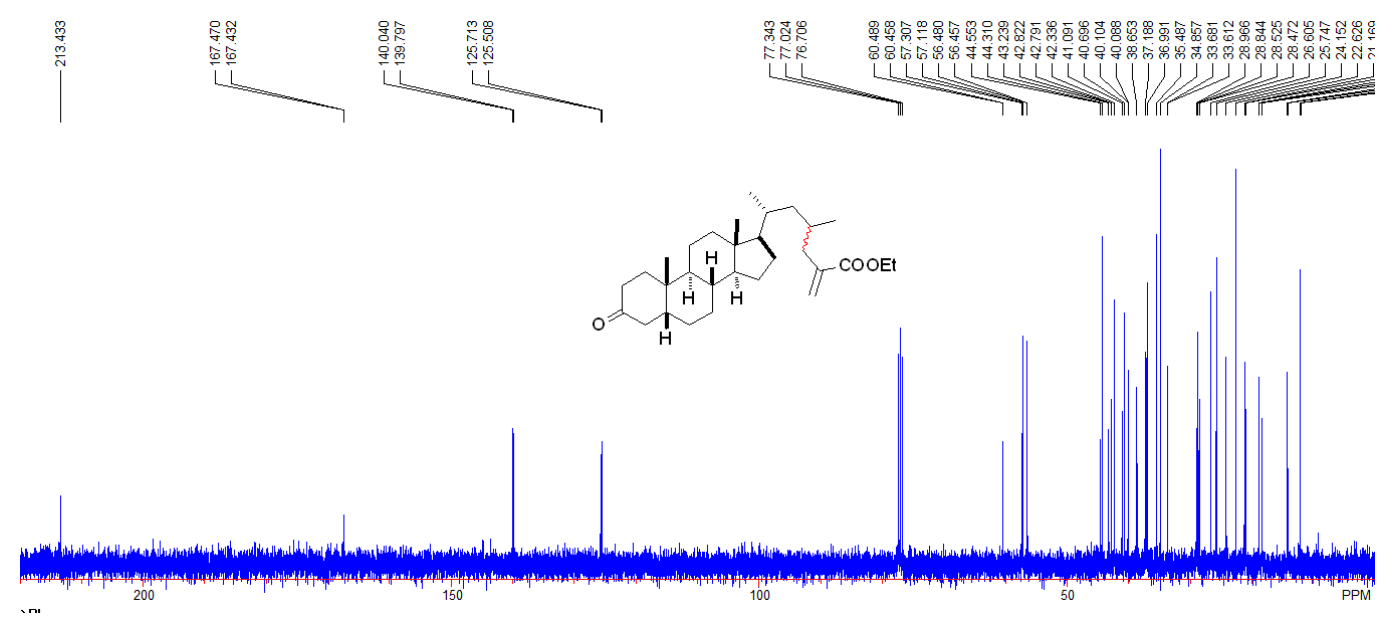
5

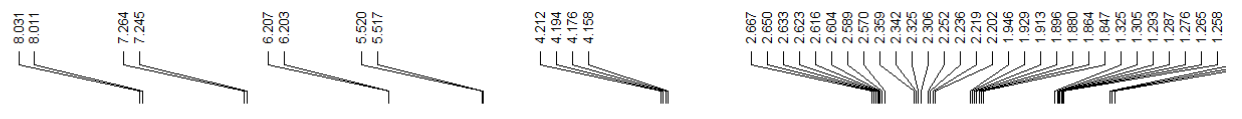

HOOC

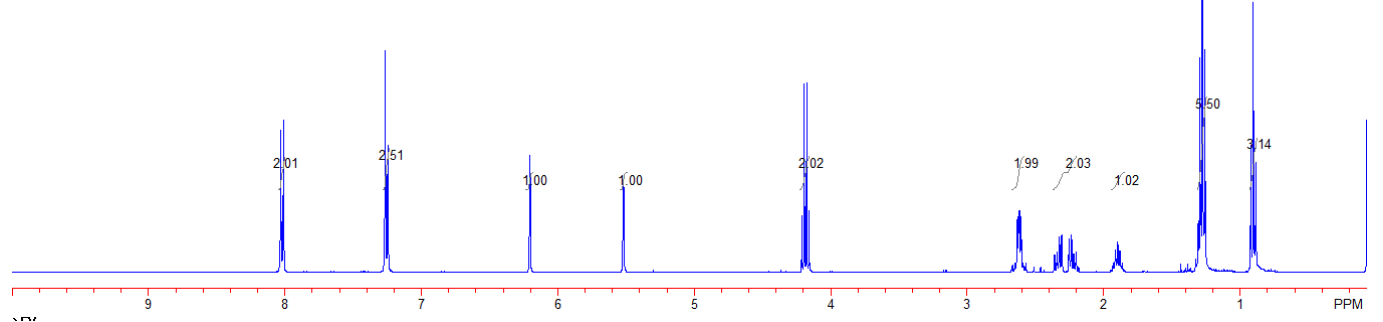

n
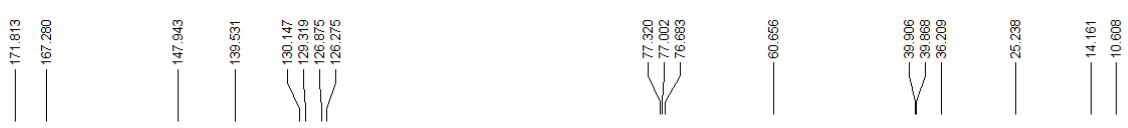

HOOC

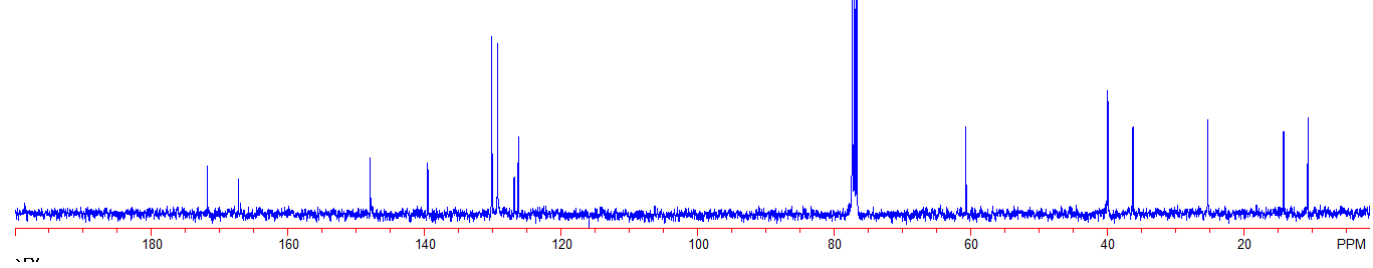




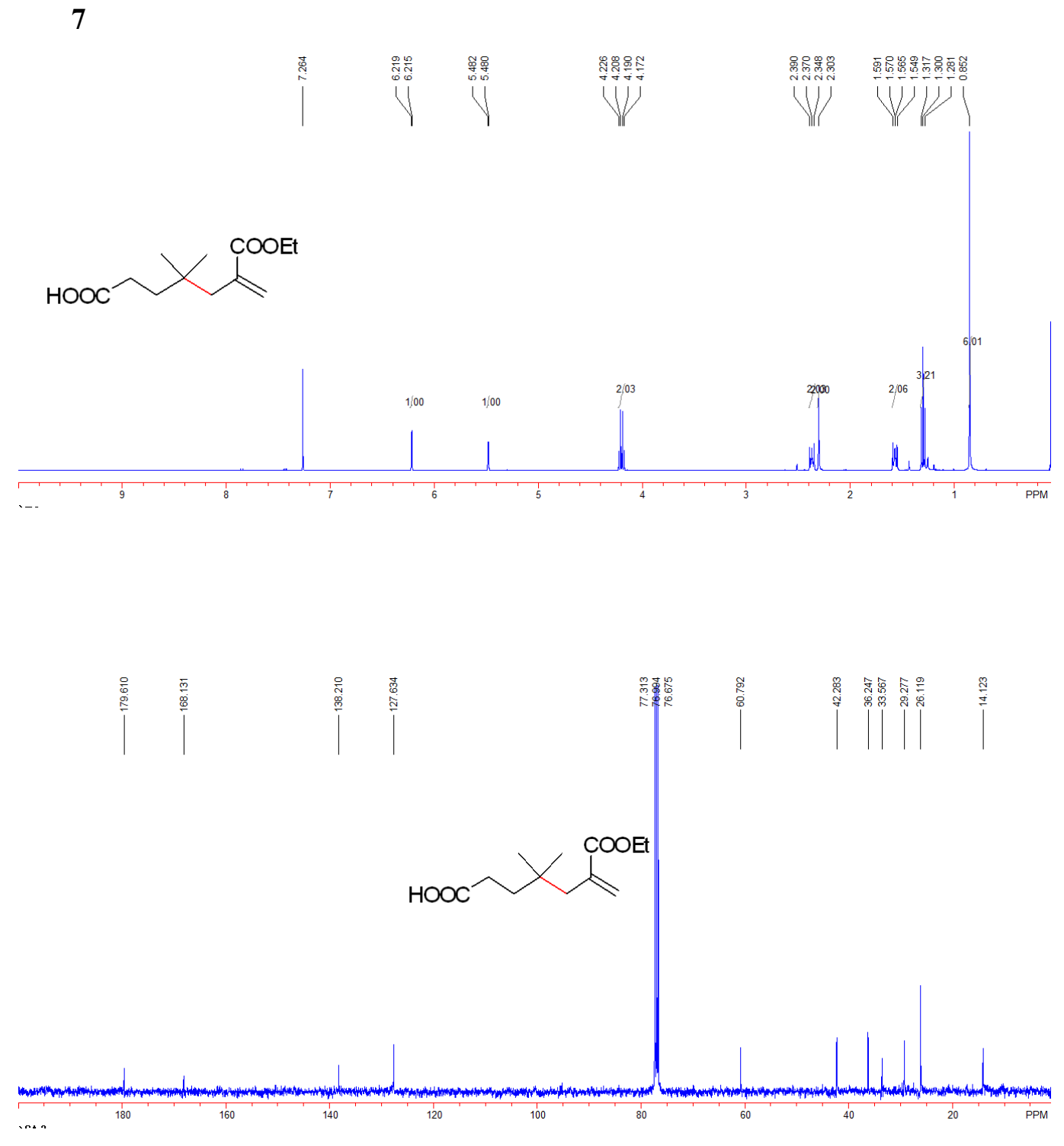



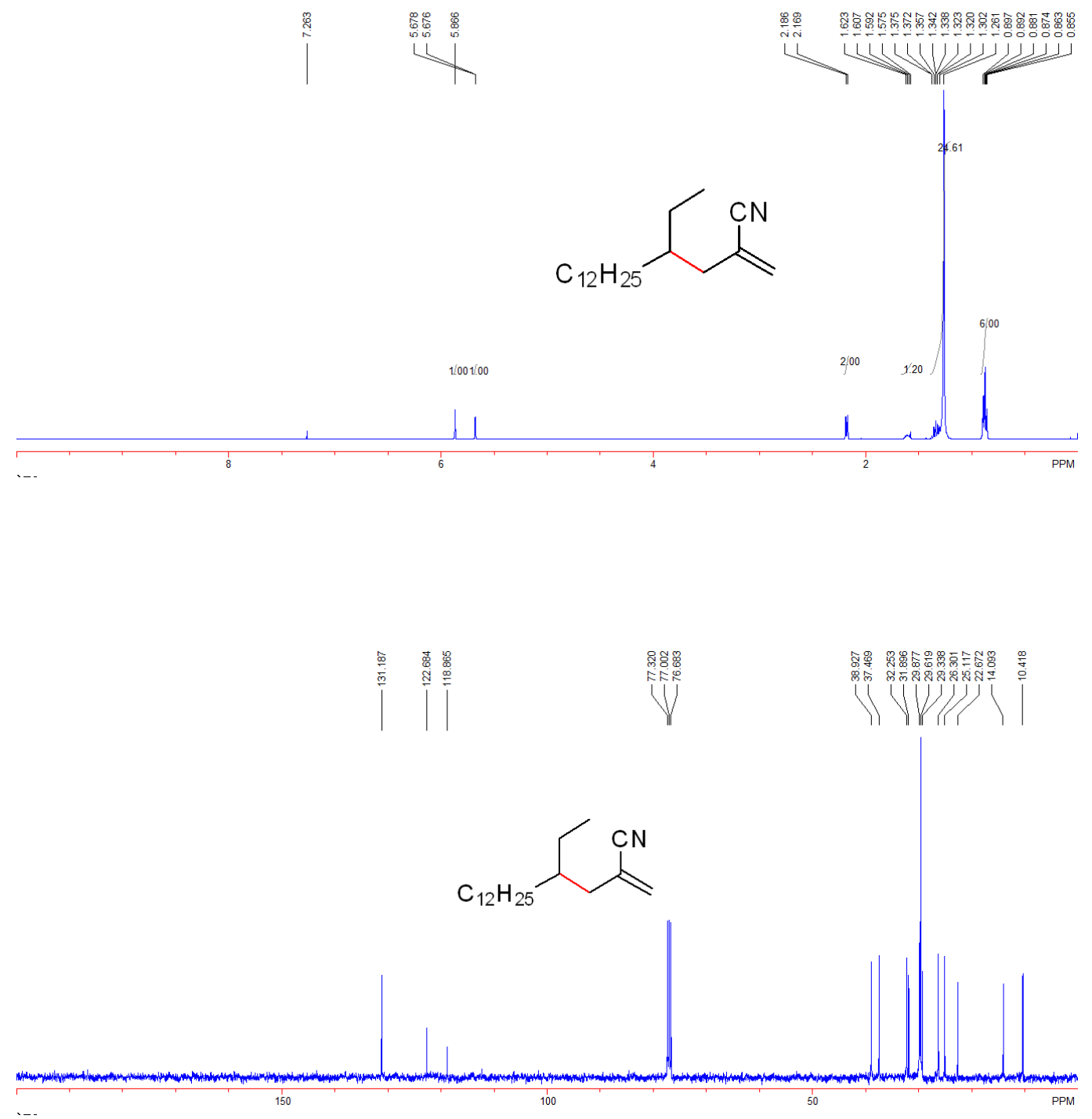
9
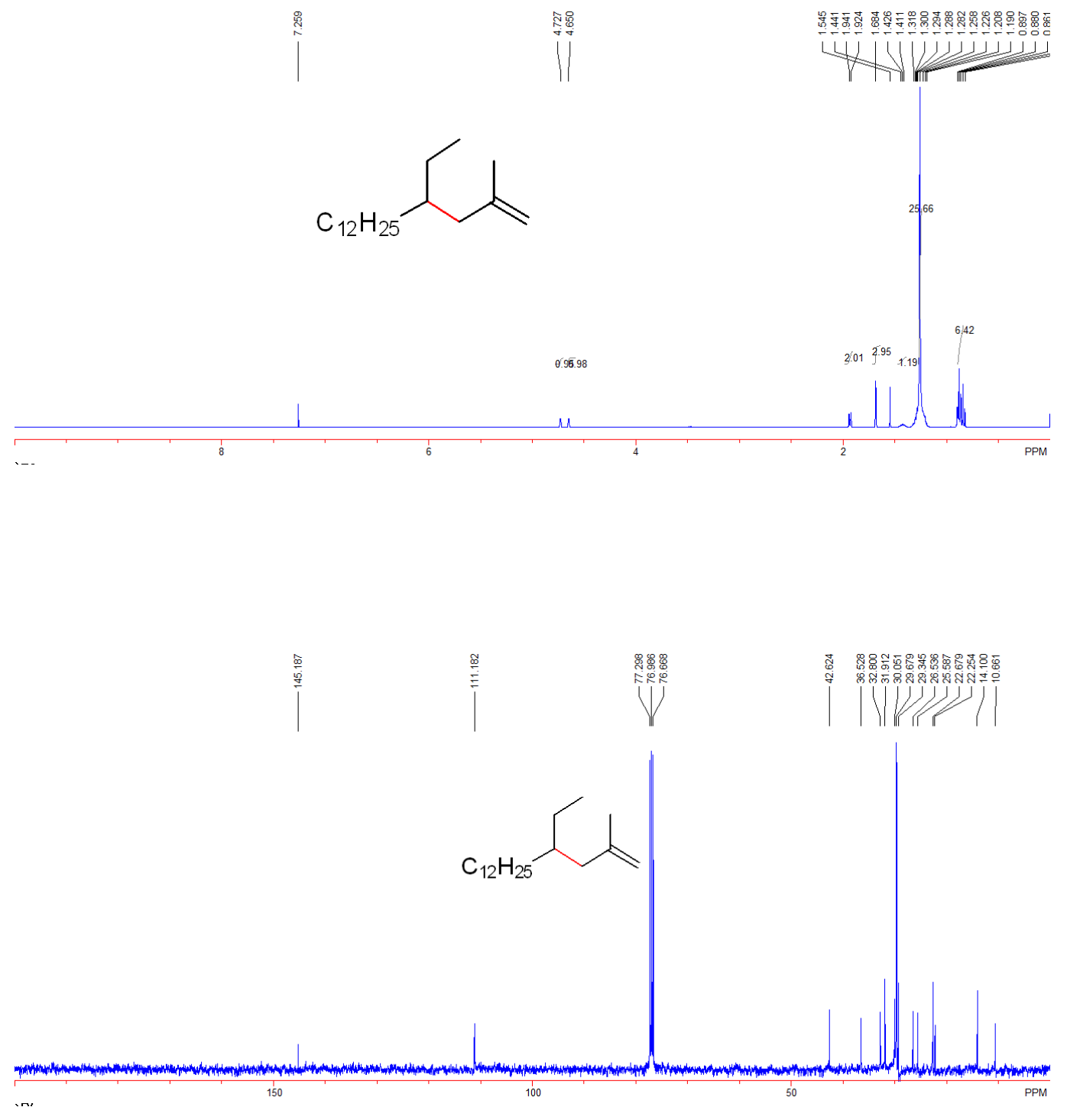
10
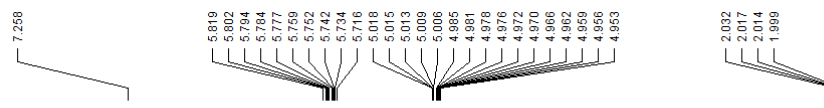

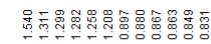
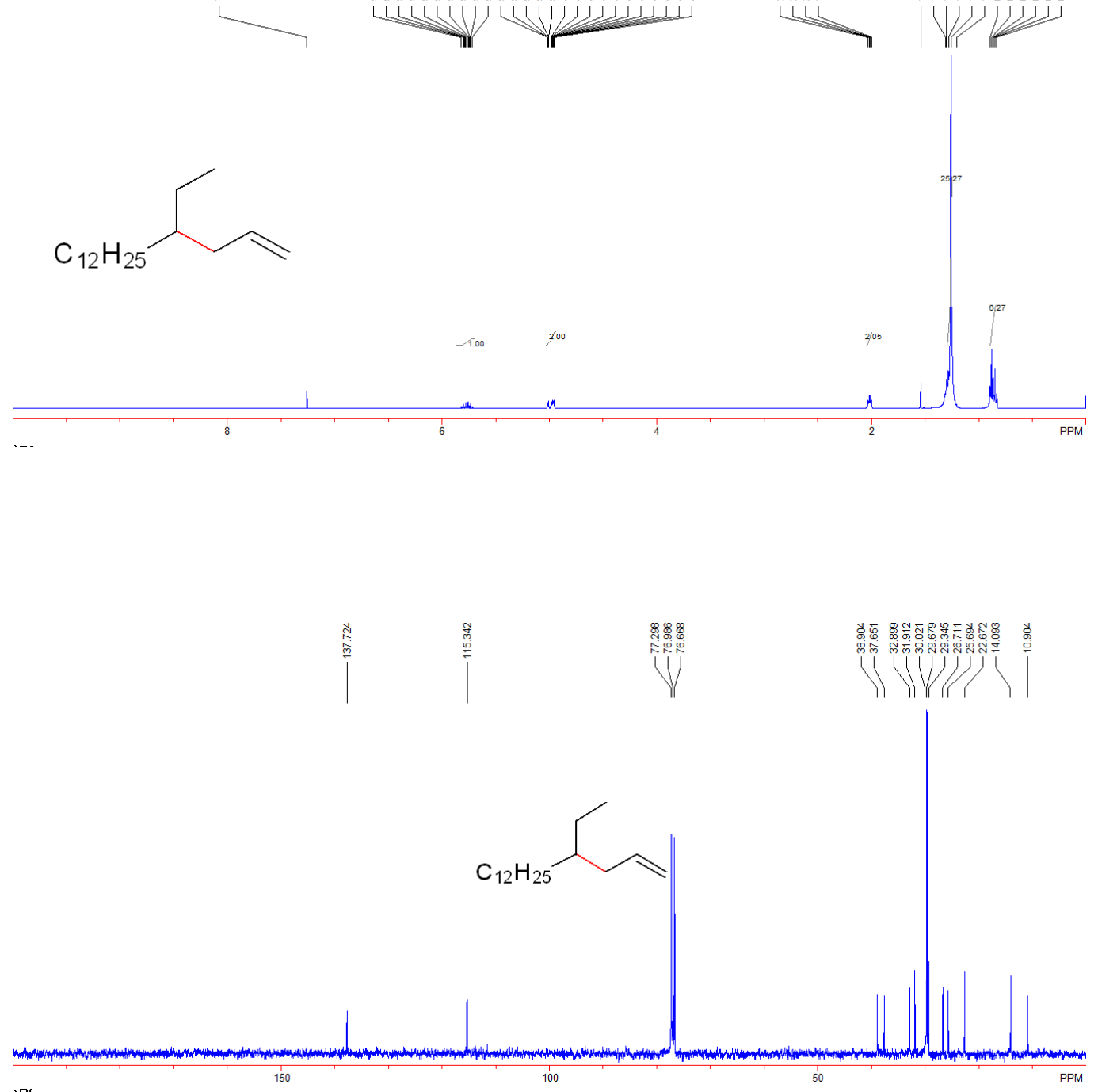
11
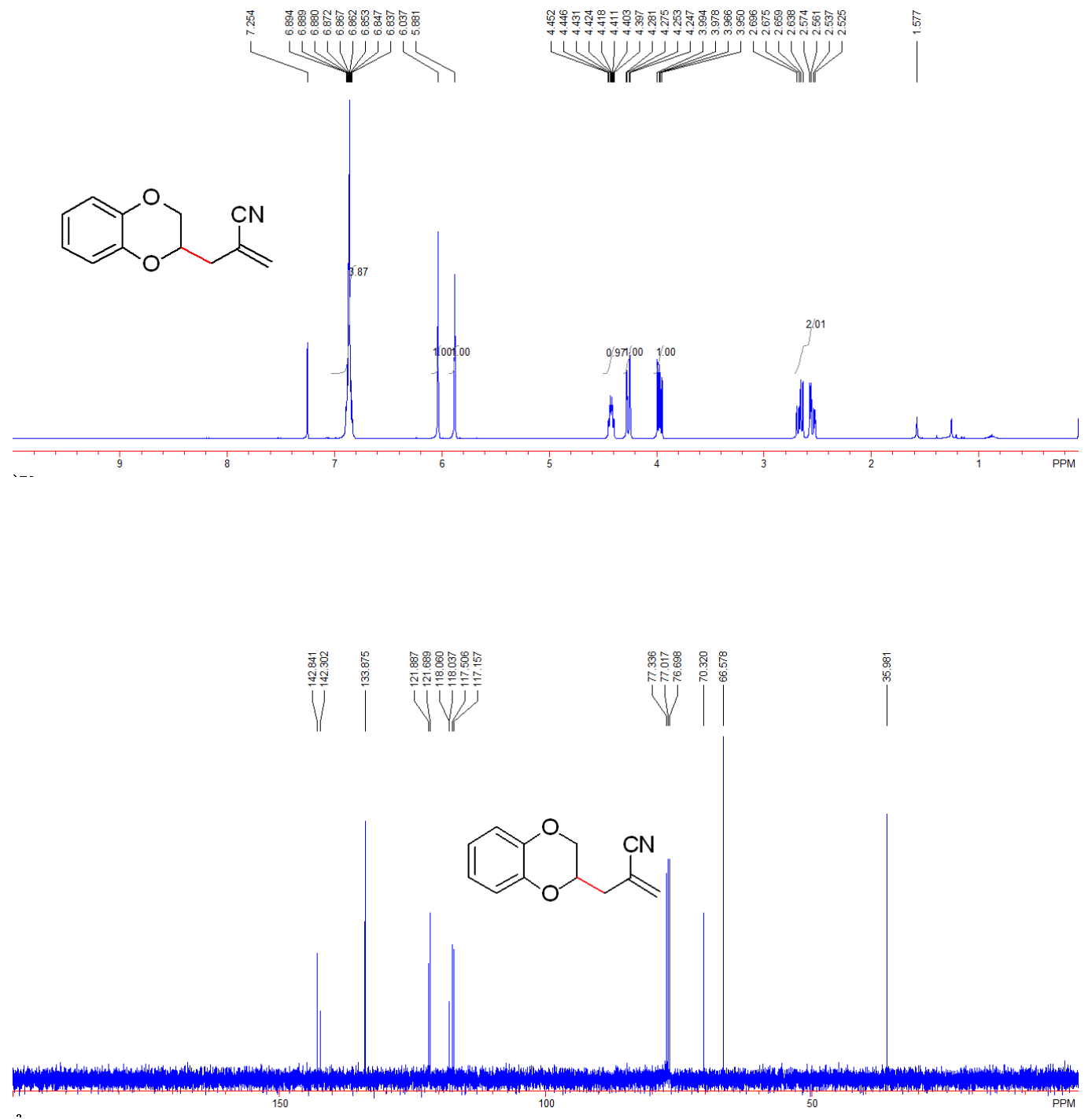
12
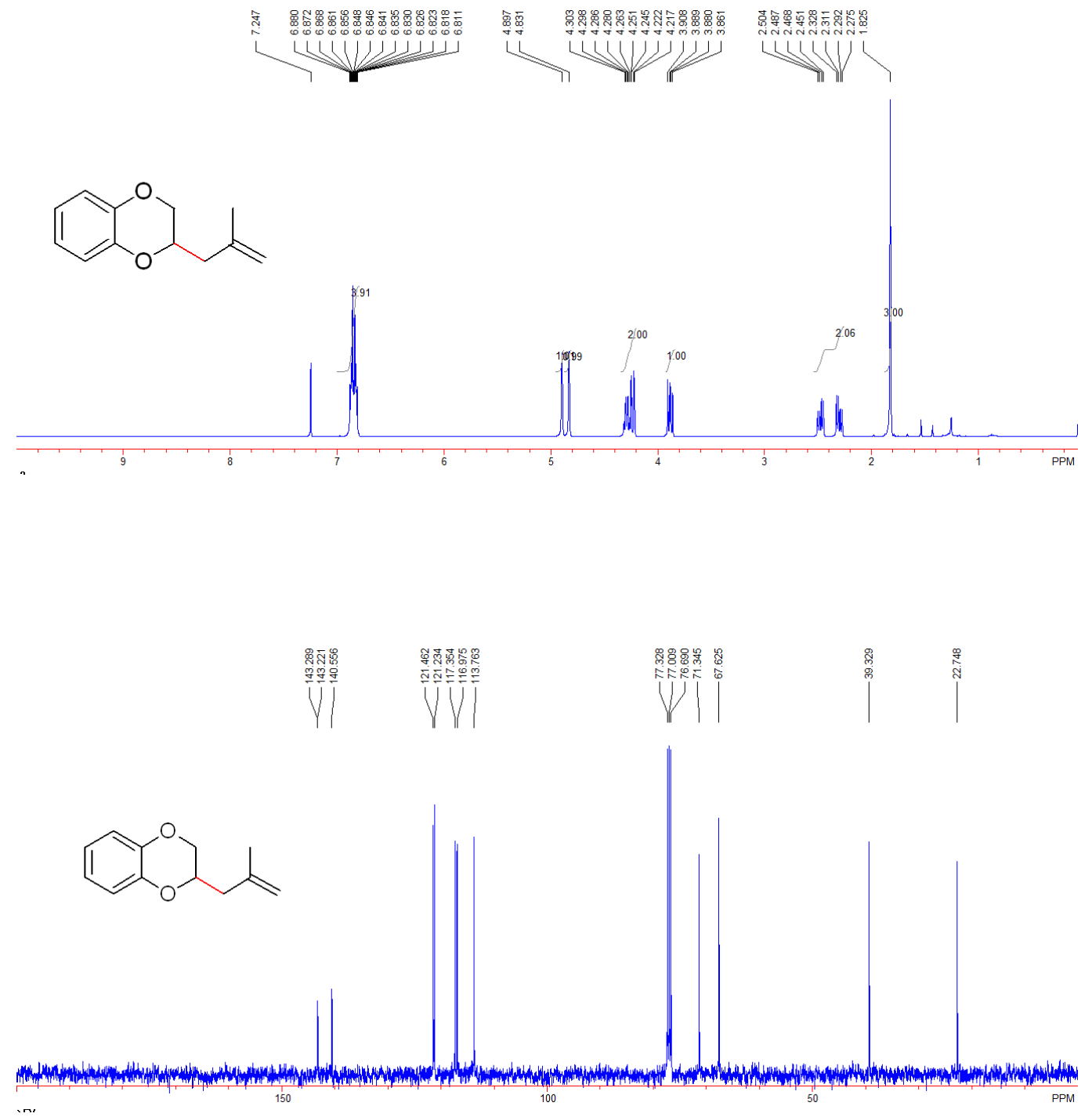
13
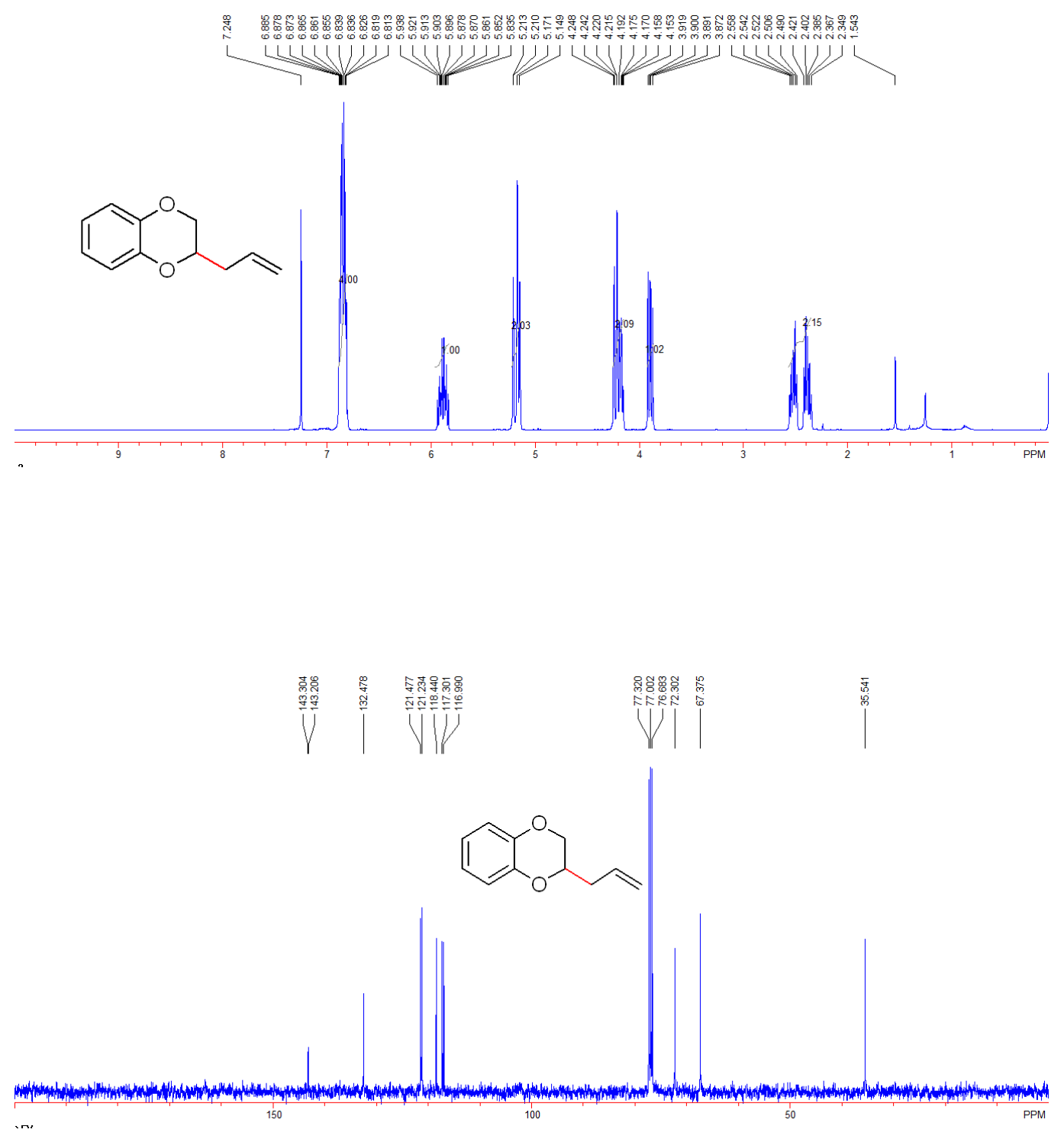
15
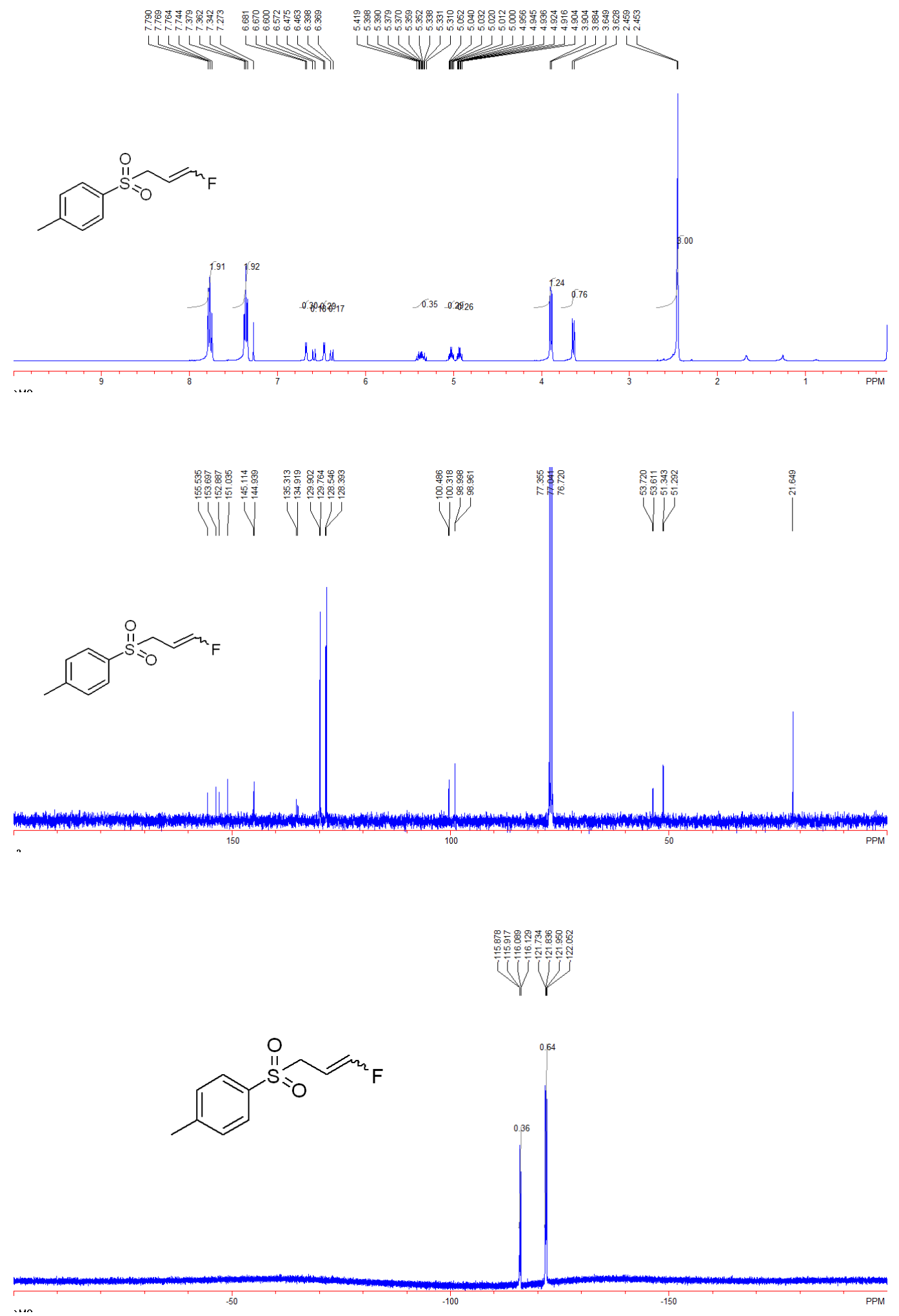


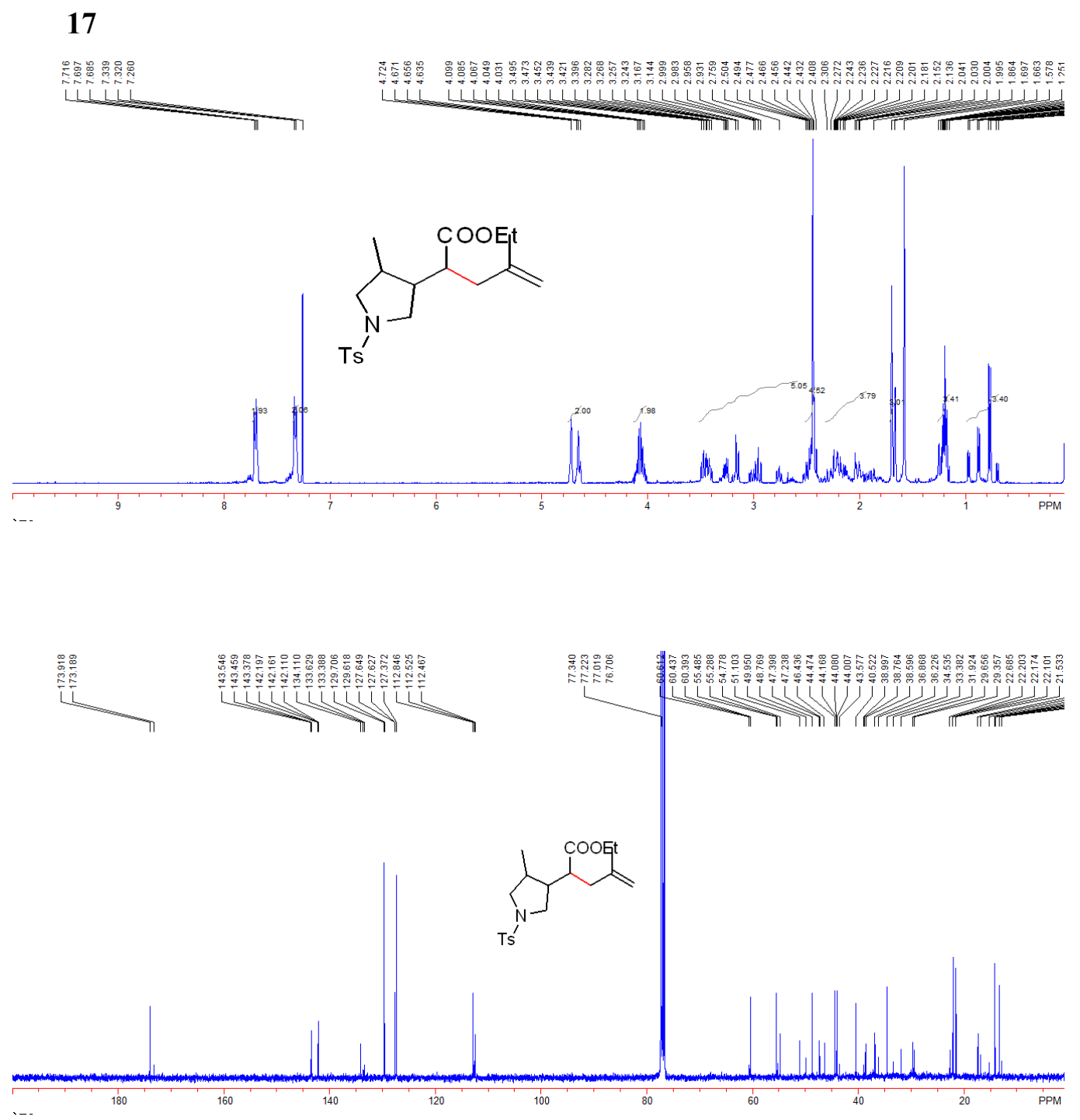

